\title{
AN APPLICATION OF PARALLEL CUT ELIMINATION IN MULTIPLICATIVE LINEAR LOGIC TO THE TAYLOR EXPANSION OF PROOF NETS
}

\author{
JULES CHOUQUET AND LIONEL VAUX AUCLAIR \\ ${ }^{a}$ Université d'Orléans, LIFO EA 4022, FR-45067 Orléans, France \\ e-mail address: jules.chouquet@univ-orleans.fr \\ URL: https://orcid.org/0000-0003-2676-0297 \\ ${ }^{b}$ Aix-Marseille Univ, CNRS, I2M, Marseille, France \\ e-mail address: lionel.vaux@univ-amu.fr \\ URL: https://orcid.org/0000-0001-9466-418X
}

\begin{abstract}
We examine some combinatorial properties of parallel cut elimination in multiplicative linear logic (MLL) proof nets. We show that, provided we impose a constraint on some paths, we can bound the size of all the nets satisfying this constraint and reducing to a fixed resultant net. This result gives a sufficient condition for an infinite weighted sum of nets to reduce into another sum of nets, while keeping coefficients finite. We moreover show that our constraints are stable under reduction.

Our approach is motivated by the quantitative semantics of linear logic: many models have been proposed, whose structure reflect the Taylor expansion of multiplicative exponential linear logic (MELL) proof nets into infinite sums of differential nets. In order to simulate one cut elimination step in MELL, it is necessary to reduce an arbitrary number of cuts in the differential nets of its Taylor expansion. It turns out our results apply to differential nets, because their cut elimination is essentially multiplicative. We moreover show that the set of differential nets that occur in the Taylor expansion of an MELL net automatically satisfies our constraints.

Interestingly, our nets are untyped: we only rely on the sequentiality of linear logic nets and the dynamics of cut elimination. The paths on which we impose bounds are the switching paths involved in the Danos-Regnier criterion for sequentiality. In order to accommodate multiplicative units and weakenings, our nets come equipped with jumps: each weakening node is connected to some other node. Our constraint can then be summed up as a bound on both the length of switching paths, and the number of weakenings that jump to a common node.
\end{abstract}

\section{INTRODUCTION}

1.1. Context: quantitative semantics and Taylor expansion. Linear logic takes its roots in the denotational semantics of $\lambda$-calculus: it is often presented, by Girard himself [Gir87], as the result of a careful investigation of the model of coherence spaces. Since its early days, linear logic has thus generated a rich ecosystem of denotational models, among which we distinguish the family of quantitative semantics. Indeed, the first ideas behind 
linear logic were exposed even before coherence spaces, in the model of normal functors [Gir88], in which Girard proposed to consider analyticity, instead of mere continuity, as the key property of the interpretation of $\lambda$-terms: in this setting, terms denote power series, representing analytic maps between modules.

This quantitative interpretation reflects precise operational properties of programs: the degree of a monomial in a power series is closely related to the number of times a function uses its argument. Following this framework, various models were considered - among which we shall include the multiset relational model as a degenerate, boolean-valued instance. These models allowed to represent and characterize quantitative properties such as the execution time [dC09], including best and worst case analysis for non-deterministic programs [LMMP13], or the probability of reaching a value [DE11]. It is notable that this whole approach gained momentum in the early 2000's, after the introduction by Ehrhard of models [Ehr02, Ehr05] in which the notion of analytic maps interpreting $\lambda$-terms took its usual sense, while Girard's original model involved set-valued formal power series. Indeed, the keystone in the success of this line of work is an analogue of the Taylor expansion formula, that can be established both for $\lambda$-terms and for linear logic proofs.

Mimicking this denotational structure, Ehrhard and Regnier introduced the differential $\lambda$-calculus [ER03] and differential linear logic [ER05], which allow to formulate a syntactic version of Taylor expansion: to a $\lambda$-term (resp. to a linear logic proof), we associate an infinite linear combination of approximants [ER08, Ehr16]. In particular, the dynamics (i.e. $\beta$-reduction or cut elimination) of those systems is dictated by the identities of quantitative semantics. In turn, Taylor expansion has become a useful device to design and study new models of linear logic, in which morphisms admit a matrix representation: the Taylor expansion formula allows to describe the interpretation of promotion - the operation by which a linear resource becomes freely duplicable - in an explicit, systematic manner. It is in fact possible to show that any model of differential linear logic without promotion gives rise to a model of full linear logic in this way [dC07]: in some sense, one can simulate cut elimination through Taylor expansion.

1.2. Motivation: reduction in Taylor expansion. There is a difficulty, however: Taylor expansion generates infinite sums and, a priori, there is no guarantee that the coefficients in these sums will remain finite under reduction. In previous works [dC07, LMMP13], coefficients were thus required to be taken in a complete semiring: all sums should converge. In order to illustrate this requirement, let us first consider the case of $\lambda$-calculus.

The linear fragment of differential $\lambda$-calculus, called resource $\lambda$-calculus, is the target of the syntactic Taylor expansion of $\lambda$-terms. In this calculus, the application of a term to another is replaced with a multilinear variant: $\langle s\rangle\left[t_{1}, \ldots, t_{n}\right]$ denotes the $n$-linear symmetric application of resource term $s$ to the multiset of resource terms $\left[t_{1}, \ldots, t_{n}\right]$. Then, if $x_{1}, \ldots, x_{k}$ denote the occurrences of $x$ in $s$, the redex $\langle\lambda x . s\rangle\left[t_{1}, \ldots, t_{n}\right]$ reduces to the sum

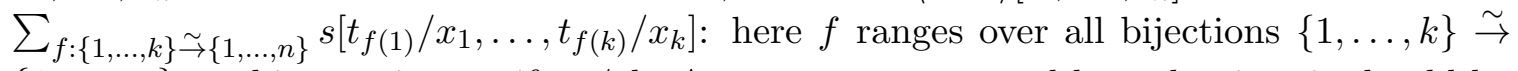
$\{1, \ldots, n\}$ so this sum is zero if $n \neq k$. As sums are generated by reduction, it should be noted that all the syntactic constructs are linear, both in the sense that they commute to sums, and in the sense that, in the elimination of a redex, no subterm of the argument multiset is copied nor erased. The key case of Taylor expansion is that of application:

$$
\mathcal{T}(M N)=\sum_{n \in N} \frac{1}{n !}\langle\mathcal{T}(M)\rangle \mathcal{T}(N)^{n}
$$




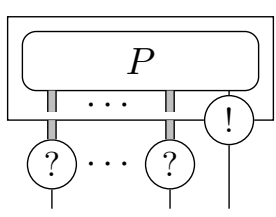

expands to

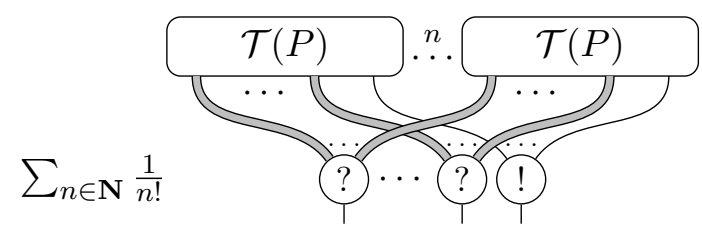

Figure 1: Taylor expansion of a promotion box (thick wires denote an arbitrary number of wires)

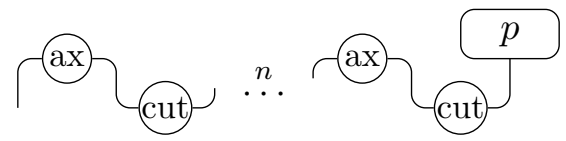

Figure 2: Example of a family of nets, all reducing to a single net $p$, by the parallel elimination of axiom cuts.

where $\mathcal{T}(N)^{n}$ is the multiset made of $n$ copies of $\mathcal{T}(N)$ - by $n$-linearity, $\mathcal{T}(N)^{n}$ is itself an infinite linear combination of multisets of resource terms appearing in $\mathcal{T}(N)$. Admitting that $\langle M\rangle\left[N_{1}, \ldots, N_{n}\right]$ represents the $n$-th derivative of $M$, computed at 0 , and $n$-linearly applied to $N_{1}, \ldots, N_{n}$, one immediately recognizes the usual Taylor expansion formula.

From (1.1), it is immediately clear that, to simulate one reduction step occurring in $N$, it is necessary to reduce in parallel in an unbounded number of subterms of each component of the expansion. Unrestricted parallel reduction, however, is ill defined in this setting. Consider the sum $\sum_{n \in \mathbf{N}}\langle\lambda x x\rangle[\cdots\langle\lambda x x\rangle[y] \cdots]$ where each summand consists of $n$ successive linear applications of the identity to the variable $y$ : then by simultaneous reduction of all redexes in each component, each summand yields $y$, so the result should be $\sum_{n \in \mathbf{N}} y$ which is not defined unless the semiring of coefficients is complete in some sense.

Those considerations apply to linear logic as well as to $\lambda$-calculus. We will use proof nets [Gir87] as the syntax for proofs of multiplicative exponential linear logic (MELL). The target of Taylor expansion is then in promotion-free differential nets [ER05], which we call resource nets in the following, by analogy with the resource $\lambda$-calculus: these form the multilinear fragment of differential linear logic.

In linear logic, Taylor expansion consists in replacing duplicable subnets, embodied by promotion boxes, with explicit copies, as in Figure 1: if we take $n$ copies of the box, the main port of the box is replaced with an $n$-ary !-link, while the ?-links at the border of the box collect all copies of the corresponding auxiliary ports. Again, to follow a single cut elimination step in $P$, it is necessary to reduce an arbitrary number of copies. And unrestricted parallel cut elimination in an infinite sum of resource nets is broken, as one can easily construct an infinite family of nets, all reducing to the same resource net $p$ in a single step of parallel cut elimination: see Figure 2.

1.3. Our approach: taming the combinatorial explosion of antireduction. The problem of convergence of series of linear approximants under reduction was first tackled by Ehrhard and Regnier, for the normalization of Taylor expansion of ordinary $\lambda$-terms [ER08]. Their argument relies on a uniformity property, specific to the pure $\lambda$-calculus: the support of the Taylor expansion of a $\lambda$-term forms a clique in some fixed coherence space of resource terms. This method cannot be adapted to proof nets: there is no coherence 
relation on differential nets such that all supports of Taylor expansions are cliques [Tas09, Section V.4.1].

An alternative method to ensure convergence without any uniformity hypothesis was first developed by Ehrhard for typed terms in a $\lambda$-calculus extended with linear combinations of terms [Ehr10]: there, the presence of sums also forbade the existence of a suitable coherence relation. This method can be generalized to strongly normalizable [PTV16], or even weakly normalizable [Vau17] terms. One striking feature of this approach is that it concentrates on the support (i.e. the set of terms having non-zero coefficients) of the Taylor expansion. In each case, one shows that, given a normal resource term $t$ and a $\lambda$-term $M$, there are finitely many terms $s$, such that:

- the coefficient of $s$ in $\mathcal{T}(M)$ is non zero; and

- the coefficient of $t$ in the normal form of $s$ is non zero.

This allows to normalize the Taylor expansion: simply normalize in each component, then compute the sum, which is component-wise finite.

The second author then remarked that the same could be done for $\beta$-reduction [Vau17], even without any uniformity, typing or normalizability requirement. Indeed, writing $s \rightrightarrows t$ if $s$ and $t$ are resource terms such that $t$ appears in the support of a parallel reduct of $s$, the size of $s$ is bounded by a function of the size of $t$ and the height of $s$. So, given that if $s$ appears in $\mathcal{T}(M)$ then its height is bounded by that of $M$, it follows that, for a fixed resource term $t$ there are finitely many terms $s$ in the support of $\mathcal{T}(M)$ such that $s \rightrightarrows t$ : in short, parallel reduction is always well-defined on the Taylor expansion of a $\lambda$-term.

Our purpose in the present paper is to develop a similar technique for MELL proof nets: we show that one can bound the size of a resource net $p$ by a function of the size of any of its parallel reducts, and of an additional quantity on $p$, yet to be defined. The main challenge is indeed to circumvent the lack of inductive structure in proof nets: in such a graphical syntax, there is no structural notion of height.

We claim that a side condition on switching paths, i.e. paths in the sense of DanosRegnier's correctness criterion [DR89], is an appropriate replacement. Backing this claim, there are first some intuitions:

- the main culprits for the unbounded loss of size in reduction are the chains of consecutive cuts, as in Figure 2;

- we want the validity of our side condition to be stable under reduction so, rather than chains of cuts, we should consider the length of switching paths;

- indeed, if $p$ reduces to $q$ via cut elimination, then the switching paths of $q$ are somehow related with those of $p$;

- and the switching paths of a resource net in $\mathcal{T}(P)$ are somehow related with those of $P$.

In the following we will establish precise formulations of those last two points: we study the structure of switching paths through cut elimination in Section 4; and we describe the switching paths of the elements of $\mathcal{T}(P)$ in Section 7.

In presence of multiplicative units, or of weakenings (nullary ?-links) and coweakenings (nullary !-links), we must also take special care of another kind of cuts, that we call evanescent cuts: when a cut between such nullary links is eliminated, it simply vanishes, leaving the rest of the net untouched, as in Figure 3, which is obviously an obstacle for our purpose. ${ }^{1}$

\footnotetext{
${ }^{1}$ The treatment of weakenings is indeed the main novelty of the present extended version over our conference paper [CVA18].
} 


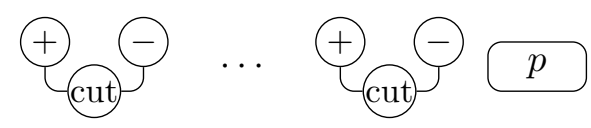

Figure 3: Evanescent cuts: here each $(+)$ node can denote a tensor unit $\mathbf{1}$ or a coweakening (a nullary !-link), and then the corresponding $(-)$ node should be the dual unit $\perp$ or a weakening (a nullary ?-link). Then the depicted net reduces to $p$ in one parallel cut elimination step.

In order to deal with nullary links, a well known trick is to attach each weakening (or $\perp$-link) to another node in the net: switching paths can then follow such jumps, which is useful to characterize exactly those nets that come from proof trees [Gir96, Appendix A.2]. Here we will rely on this structure to control the effect of eliminating evanescent cuts on the size of a net.

In all our exposition, we adopt a particular presentation of nets: we consider $n$-ary exponential links rather than separate (co)dereliction and (co)contraction, as this allows to reduce the dynamics of resource nets to that of multiplicative linear logic (MLL) proof nets. ${ }^{2}$

1.4. Outline. In Section 2, we first introduce MLL proof nets formally, in the term-based syntax of Ehrhard [Ehr14]. We define the parallel cut elimination relation $\rightrightarrows$ in this setting, that we decompose into multiplicative reduction $\rightrightarrows m$, axiom-cut reduction $\rightrightarrows a$ and evanescent reduction $\rightrightarrows e$. We also present the notion of switching path for this syntax, and introduce the two quantities that will be our main objects of study in the following:

- the maximum number $\mathbf{j d}(p)$ of $\perp$-links that jump to a common target;

- the maximum length $\ln (p)$ of any switching path in the net $p$.

Let us mention that typing plays absolutely no role in our approach, so we do not even consider formulas of linear logic in our exposition: we will rely on the geometrical structure of nets only.

We show in Section 3 that, if $p \rightrightarrows_{m} q, p \rightrightarrows_{a} q$ or $p \rightrightarrows_{e} q$ then the size of $p$ is bounded by a function of $\ln (p), \mathbf{j d}(p)$, and the size of $q$. In order to be able to iterate this combinatorial argument, we must show that, given bounds for $\ln (p)$ and $\mathbf{j d}(p)$, we can infer bounds on $\ln (q)$ and $\mathbf{j d}(q)$ : this is the subject of Sections 4 and 5 .

Section 4 is dedicated to the proof that we can bound $\ln (q)$ by a function of $\ln (p)$ : the main case is the multiplicative reduction, as this may create new switching paths in $q$ that we must relate with those in $p$. In this task, we concentrate on the notion of slipknot: a pair of residuals of a cut of $p$ occurring in a path of $q$. Slipknots are essential in understanding how switching paths are structured after cut elimination: this analysis is motivated by a technical requirement of our approach, but it can also be considered as a contribution to the theory of MLL nets per se.

In Section 5 , we show that $\mathbf{j} \mathbf{d}(q)$ is bounded by a function of $\ln (p)$ and $\mathbf{j d}(p)$ : the critical case here is that of chains of jumps between evanescent cuts.

We leverage all of the above results in Section 6 , to generalize them to a reduction $p \rightrightarrows q$, or even an arbitrary sequence of reductions. In particular, if $p \rightrightarrows q$ then the size

\footnotetext{
${ }^{2}$ In other words, we adhere to a version of linear logic proof nets and resource nets which is sometimes called nouvelle syntaxe, although it dates back to Regnier's PhD thesis [Reg92]. For the linear logic connoisseur, this is already apparent in Figure 1. See also the discussion in our conclusion (Section 8).
} 


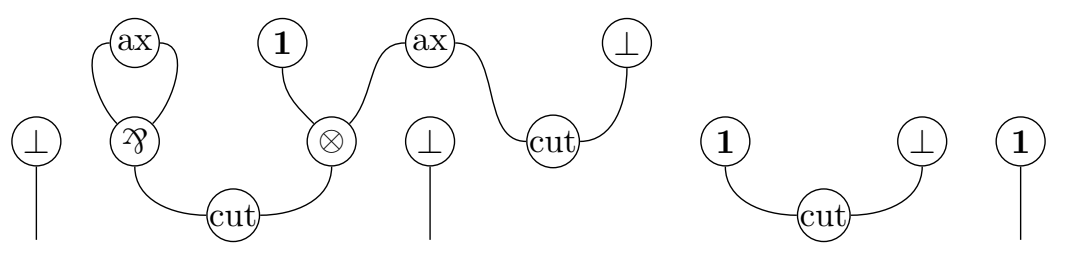

Figure 4: An example of multiplicative net

of $p$ is bounded by a function of the size of $q$ and of $\ln (p)$ and $\mathbf{j d}(p)$. Again, this result is motivated by the study of quantitative semantics, but it is essentially a theorem about MLL.

We establish the applicability of our approach to the Taylor expansion of MELL proof nets in Section 7: we show that if $p$ is a resource net of $\mathcal{T}(P)$, then $\ln (p)$ is bounded by a function of the size of $P$, and $\mathbf{j d}(p)$ is bounded by the size of $P$.

Finally, we discuss the scope of our results in the concluding Section 8.

\section{Definitions}

We provide here the minimal definitions necessary for us to work with MLL proof nets. As stated before, let us stress the fact that the choice of MLL is not decisive for the development of Sections 2 to 6 . The reader can check that we rely on three ingredients only:

- the definition of switching paths;

- the fact that multiplicative reduction amounts to plug bijectively the premises of a $\otimes$-link with those of 2 -link (in the nullary case, evanescent cuts simply vanish);

- the definition of jumps and how they are affected by cut elimination.

The results of those sections are thus directly applicable to resource nets, thanks to our choice of generalized exponential links: this will be done in Section 7.

2.1. Nets. A proof net is usually presented as a graphical object such as that of Figure 4 . Following Ehrhard [Ehr14, Ehr16], we will rely on a term syntax for denoting such nets. This is based on a quite standard trichotomy: a proof net can be divided into a top layer of axioms, followed by trees of connectives, down to cuts between the conclusions of some trees.

We will represent the conclusions of axiom rules by variables: the duality between two conclusions of an axiom rule is given by an involution $x \mapsto \bar{x}$ over the set $\mathbf{V}$ of variables. Our nets will be finite families of trees and cuts, where trees are inductively generated from variables by the application of MLL connectives, of arbitrary arity: $\otimes\left(t_{1}, \ldots, t_{n}\right)$ and $8\left(t_{1}, \ldots, t_{n}\right)$. A tree thus represents a conclusion of a net, together with the nodes above it, up to axiom conclusions. A cut is then given by the pair of trees $\left\langle t_{1} \mid t_{2}\right\rangle$, whose conclusions it cuts together. In order to distinguish between various occurrences of nullary connectives $\mathbf{1}=\otimes()$ and $\perp=\not \supset()$, we will index them with labels taken from sets $\mathbf{U}_{\mathbf{1}}$ and $\mathbf{U}_{\perp}$.

Formally, the set of raw trees (denoted by $s$, $t$, etc.) is generated as follows:

$$
t::=x\left|\mathbf{1}_{\lambda}\right| \perp_{\mu}\left|\otimes\left(t_{1}, \ldots, t_{n}\right)\right| \not \partial\left(t_{1}, \ldots, t_{n}\right)
$$

where $x$ ranges over $\mathbf{V}, \lambda$ ranges over $\mathbf{U}_{\mathbf{1}}, \mu$ ranges over $\mathbf{U}_{\perp}$ and we require $n \neq 0$ in the two last cases. We assume $\mathbf{V}, \mathbf{U}_{\mathbf{1}}$ and $\mathbf{U}_{\perp}$ are pairwise disjoint and all three are denumerably infinite. We will always identify a nullary connective tree $\mathbf{1}_{\lambda}$ or $\perp_{\mu}$ with its label $\lambda$ or $\mu$, so that $\mathbf{A}=\mathbf{V} \cup \mathbf{U}_{\mathbf{1}} \cup \mathbf{U}_{\perp}$ is just the set of atomic trees. We will generally use letters $x, y, z$ 
for variables, $\mu$ for the elements of $\mathbf{U}_{\perp}, \lambda$ for the elements of $\mathbf{U}_{\mathbf{1}}$, and $s, t, u, v$ for arbitrary raw trees.

We write $\mathbf{T}(t)$ for the set of subtrees of a given raw tree $t$, which is defined inductively in the natural way: if $t \in \mathbf{A}$, then $\mathbf{T}(t)=\{t\}$; if $t=\diamond\left(t_{1}, \ldots, t_{n}\right)$ with $\diamond \in\{\otimes, \not 8\}$, then $\mathbf{T}(t)=\{t\} \cup \bigcup_{i \in\{1, \ldots, n\}} \mathbf{T}\left(t_{i}\right)$. We moreover write $\mathbf{V}(t)$ for $\mathbf{T}(t) \cap \mathbf{V}$, and similarly for $\mathbf{U}_{\mathbf{1}}(t)$, $\mathbf{U}_{\perp}(t)$ and $\mathbf{A}(t)$. A tree is then a raw tree $t$ such that if $\diamond\left(t_{1}, \ldots, t_{n}\right) \in \mathbf{T}(t)$ then the sets $\mathbf{A}\left(t_{i}\right)$ for $1 \leq i \leq n$ are pairwise disjoint: in other words, each atom occurs at most once in $t$. As a consequence, each subtree $u \in \mathbf{T}(t)$ occurs exactly once in a tree $t$.

A cut is an unordered pair $c=\langle t \mid s\rangle$ of trees such that $\mathbf{A}(t) \cap \mathbf{A}(s)=\emptyset$, and then we set $\mathbf{T}(c)=\mathbf{T}(t) \cup \mathbf{T}(s)$, and similarly for $\mathbf{V}(c), \mathbf{U}_{\mathbf{1}}(c), \mathbf{U}_{\perp}(c)$ and $\mathbf{A}(c)$. Note that, in the absence of typing, we do not put any compatibility requirement on cut trees.

Given a set $A$, we denote by $\vec{a}$ any finite family $\left(a_{i}\right)_{i \in I} \in A^{I}$ of elements of $A$. In general, we abusively identify $\vec{a}$ with any enumeration $\left(a_{1}, \ldots, a_{n}\right) \in A^{n}$ of its elements, and we may even write $\vec{a}=a_{1}, \ldots, a_{n}$ in this case; moreover, we simply write $\vec{a}, \vec{b}$ for the concatenation of families $\vec{a}$ and $\vec{b}$ (whose index set is implicitly the sum of the index sets of $\vec{a}$ and $\vec{b}$ ). We may also write, e.g., $a_{i} \in \vec{a}$, identifying the family $\vec{a}$ with its support set. Since we only consider families of pairwise distinct elements, such abuse of notation is generally harmless: in this case, the only difference between $\left(a_{i}\right)_{i \in I}$ and its support set $\left\{a_{i} \mid i \in I\right\}$ is whether the bijection $i \mapsto a_{i}$ is part of the data or not. If $f$ is a function from $A$ to any powerset, we extend it to families in the obvious way, setting $f(\vec{a})=\bigcup_{a \in a} f(a)$. E.g., if $\vec{\gamma}$ is a family of trees or cuts we write $\mathbf{V}(\vec{\gamma})=\bigcup_{\gamma \in \vec{\gamma}} \mathbf{V}(\gamma)$.

An MLL bare proof net is a pair $p=(\vec{c} ; \vec{t})$ of a finite family $\vec{c}$ of pairwise distinct cuts and a finite family $\vec{t}$ of pairwise distinct trees such that: for all distinct cuts or trees $\gamma, \gamma^{\prime} \in \vec{c} \cup \vec{t}, \mathbf{A}(\gamma) \cap \mathbf{A}\left(\gamma^{\prime}\right)=\emptyset$; and $\mathbf{V}(p)=\mathbf{V}(\vec{c}) \cup \mathbf{V}(\vec{t})$ is closed under the involution $x \mapsto \bar{x}$. We write $\mathbf{C}(p)=\vec{c}$ for the family of cuts of $p$. For any tree, cut or bare proof net $\gamma$, we define the size of $\gamma$ as $\operatorname{size}(\gamma)=\# \mathbf{T}(\gamma)$ : graphically, $\operatorname{size}(p)$ is nothing but the number of wires in $p$.

Remark 2.1. In a graphical structure such as that of Figure 4, the interface (i.e. the set of extremities of dangling wires, which represent the conclusions of the net) is relevant: in particular, cut elimination preserves this interface. So, in $p=(\vec{c} ; \vec{t}), \vec{t}$ is intrinsically a family, whose index set is precisely the interface of the structure.

On the other hand, the rest of the net should be considered up to isomorphism: in our case, this amounts to the reindexing of cuts, and the renaming of atoms, preserving the duality involution on variables. We may call $\alpha$-equivalence the corresponding equivalence relation on bare proof nets, as it has the very same status as the renaming of bound variables in the ordinary $\lambda$-calculus. In particular, $\vec{c}$ should be considered as a set, although we introduce it as a family here, just because it will be convenient to treat the concatenation $\vec{c}, \vec{t}$ as a family of cuts and trees in the following.

The reader may check that bare proof nets quotiented by $\alpha$-equivalence, as introduced above, are exactly the usual (untyped) proof structures for MLL (with connectives of arbitrary arity). We keep this quotient implicit whenever possible in the remaining: in any case, $\alpha$-equivalence preserves the size of nets, as well as the length of paths to be introduced later. $^{3}$

\footnotetext{
${ }^{3}$ Note that this situation differs slightly from the case of interaction nets [Laf90], where explicit axioms and cuts links are missing and there is no top-down orientation a priori. Term syntaxes have been proposed
} 


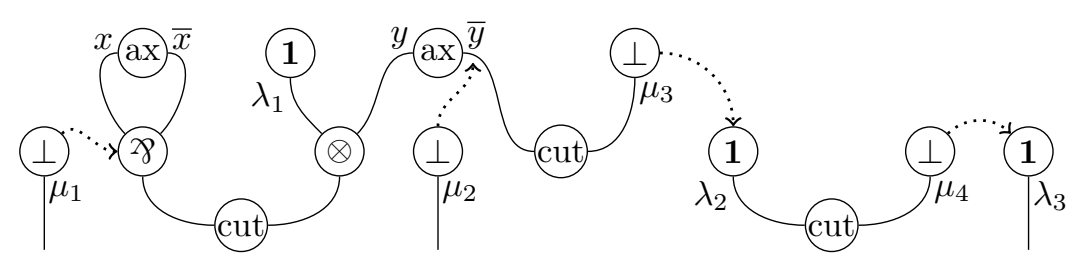

Figure 5: The net $p_{0}=\left(\left\langle\not \gamma(x, \bar{x}) \mid \otimes\left(\mathbf{1}_{\lambda_{1}}, y\right)\right\rangle,\left\langle\bar{y} \mid \perp_{\mu_{3}}\right\rangle,\left\langle\mathbf{1}_{\lambda_{2}} \mid \perp_{\mu_{4}}\right\rangle ; \perp_{\mu_{1}}, \perp_{\mu_{2}}, \mathbf{1}_{\lambda_{3}}\right)$ with $\jmath_{p_{0}}$ : $\mu_{1} \mapsto \mathcal{X}(x, \bar{x}), \mu_{2} \mapsto \bar{y}, \mu_{3} \mapsto \lambda_{2}, \mu_{4} \mapsto \lambda_{3}$.

As announced in our introduction, our nets will be equipped with jumps from $\perp$ nodes to other nodes. An MLL proof net will thus be the data of a bare proof net $p$ and of a jump function $\jmath: \mathbf{U}_{\perp}(p) \rightarrow \mathbf{T}(p)$. We will often identify a proof net with its underlying bare net $p$, and then write $\jmath_{p}$ for the associated jump function. Figure 5 presents such a net, whose underlying graphical structure is that of Figure 4.

We can already introduce the first of our two key quantities: the jump degree $\mathbf{j} \mathbf{d}(p)$ of a net $p$. We first define the jump degree of any tree $t \in \mathbf{T}(p)$, setting $\mathbf{j} \mathbf{d}_{p}(t)=\#\left\{\mu \in \mathbf{U}_{\perp}(p) \mid\right.$ $\left.\jmath_{p}(\mu)=t\right\}$. We will often write $\mathbf{j d}(t)$ instead of $\mathbf{j d}_{p}(t)$ if $p$ is clear from the context. Then we set $\mathbf{j d}(p)=\max \{\mathbf{j d}(t) \mid t \in \mathbf{T}(p)\}$.

Remark 2.2. Originally, jumps were introduced as pis aller for the characterization of sequentializable proof nets [Gir96, Appendix A.2]. Indeed, in presence of multiplicative units and without jumps, Danos-Regnier's correctness criterion, requiring the connectedness and acyclicity of switching graphs, fails to accept some proof nets corresponding to proof trees using the $\perp$-rule. So, to characterize all sequentializable nets, one has to require the existence of a jump function that makes all the switching graphs connected and acyclic. This additional structure is somewhat arbitrary, and it restores a form of bureaucratic sequentiality: distinct jumping functions on the same bare net may yield equivalent sequentializations. And there is no satisfactory solution to that issue: if a notion of proof net could capture proof equivalence in MLL with units, then deciding the identity of proof nets in that setting would not be tractable, simply because proof equivalence is PSPACE-complete [HH16].

A simple, consensual alternative is to forget about jumps and drop the connectedness requirement: the acyclicity criterion characterizes exactly those nets that are sequentializable using an additional mix-rule, corresponding to the parallel juxtaposition of nets. In particular, this weaker requirement is sufficient to avoid the problematic cases of cut elimination. This is the approach we adopt: after defining switchings and paths in Subsection 2.3, we will restrict our attention to acyclic nets only.

We associate jump functions with nets nonetheless, but for a different purpose: bounding the jump degree in a net will allow us to control the combinatorics of the elimination of evanescent cuts, in situations such as that of Figure 3. Since switching paths can follow jumps, and those paths will be our main focus throughout the remaining of the paper, we chose to consider proof nets as equipped with jumps by default. Still, the reader should be aware that the main subject of interest is the underlying structure of bare proof nets.

for those [MS08, FM99, among others] but the correspondence is less immediate: it must be restricted to deadlock-free interaction nets and, in addition to $\alpha$-equivalence, one must introduce some mechanism to deal with implicit axiom-cut elimination in the application of reduction rules. 
2.2. Cut elimination. A reducible cut is a cut $\langle t \mid s\rangle$ such that:

- $t$ is a variable and $\bar{t} \notin \mathbf{V}(s)$ (axiom cut);

- or $t \in \mathbf{U}_{\mathbf{1}}$ and $s \in \mathbf{U}_{\perp}$, and $\jmath(s) \notin\{t, s\}$ (evanescent cut);

- or we can write $t=\otimes\left(t_{1}, \ldots, t_{n}\right)$ and $s=8\left(s_{1}, \ldots, s_{n}\right)$ (multiplicative cut).

The substitution $\gamma[t / x]$ of a tree $t$ for a variable $x$ in a tree (or cut, or family of trees and/or cuts) $\gamma$ is defined in the usual way, with the additional assumption that $\mathbf{A}(t)$ and $\mathbf{A}(\gamma)$ are disjoint. By the definition of trees, this substitution is essentially linear: each variable $x$ appears at most once in $\gamma$.

There are three basic cut elimination steps defined for bare proof nets, one for each kind of reducible cut:

- the elimination of a multiplicative cut yields a family of cuts: we write

$$
\left\langle\otimes\left(t_{1}, \ldots, t_{n}\right) \mid \gamma \gamma\left(s_{1}, \ldots, s_{n}\right)\right\rangle \rightarrow_{m}\left\langle t_{1} \mid s_{1}\right\rangle, \ldots,\left\langle t_{n} \mid s_{n}\right\rangle
$$

that we extend to nets by setting $(c, \vec{c} ; \vec{t}) \rightarrow_{m}\left(\vec{c}^{\prime}, \vec{c} ; \vec{t}\right)$ whenever $c \rightarrow_{m} \vec{c}^{\prime}$;

- the elimination of an axiom cut generates a substitution: we write $(\langle x \mid s\rangle, \vec{c} ; \vec{t}) \rightarrow_{a}$ $(\vec{c} ; \vec{t})[s / \bar{x}]$ whenever $\bar{x} \notin \mathbf{V}(s)$;

- the elimination of an evanescent cut just deletes that cut: we write $(\langle\lambda \mid \mu\rangle, \vec{c} ; \vec{t}) \rightarrow_{e}$ $(\vec{c} ; \vec{t})$ whenever $\jmath_{p}(\mu) \notin\{\mu, \lambda\} .4$

Then we write $p \rightarrow p^{\prime}$ if $p \rightarrow_{m} p^{\prime}$ or $p \rightarrow_{a} p^{\prime}$ or $p \rightarrow_{e} p^{\prime}$. Observe that if $p \rightarrow p^{\prime}$ then $\mathbf{A}\left(p^{\prime}\right) \subseteq \mathbf{A}(p)$.

In order to define cut elimination between proof nets (and not bare proof nets only), we need to modify the jump function. Indeed, assume $p=(\langle t \mid s\rangle, \vec{c} ; \vec{t})$ and $p^{\prime}$ is obtained from $p$ by reducing the cut $\langle t \mid s\rangle$. Then $\mathbf{U}_{\perp}\left(p^{\prime}\right) \subseteq \mathbf{U}_{\perp}(p)$, but if $\mu \in \mathbf{U}_{\perp}\left(p^{\prime}\right)$ and $\jmath_{p}(\mu)=t$, we need to redefine $\jmath_{p^{\prime}}(\mu)$, as in general $t \notin \mathbf{T}\left(p^{\prime}\right)$. This is done as follows:

- if $\langle t \mid s\rangle=\left\langle\otimes\left(t_{1}, \ldots, t_{n}\right) \mid \gamma\left(s_{1}, \ldots, s_{n}\right)\right\rangle$ then for all $\mu \in \mathbf{U}_{\perp}(p)=\mathbf{U}_{\perp}\left(p^{\prime}\right)$ such that $\jmath_{p}(\mu)=$ $\otimes\left(t_{1}, \ldots, t_{n}\right)\left(\operatorname{resp} . \not \partial\left(s_{1}, \ldots, s_{n}\right)\right)$, we set $\jmath_{p^{\prime}}(\mu)=t_{1}\left(\right.$ resp. $\left.s_{1}\right) ;^{5}$

- if $\langle t \mid s\rangle=\langle t \mid x\rangle$ and $p^{\prime}$ is obtained from $p$ by substituting $t$ for $\bar{x}$, then for all $\mu \in \mathbf{U}_{\perp}(p)=$ $\mathbf{U}_{\perp}\left(p^{\prime}\right)$ such that $\jmath_{p} \in\{x, \bar{x}\}$, we set $\jmath_{p^{\prime}}(\mu)=t$;

- if $\langle t \mid s\rangle=\langle\mu \mid \lambda\rangle$, then for all $\mu^{\prime} \in \mathbf{U}_{\perp}\left(p^{\prime}\right)=\mathbf{U}_{\perp}(p) \backslash\{\mu\}$ such that $\jmath_{p}\left(\mu^{\prime}\right) \in\{\mu, \lambda\}$, we set $\jmath_{p^{\prime}}\left(\mu^{\prime}\right)=\jmath_{p}(\mu)$

The result of eliminating the multiplicative cut (resp. axiom cut; evanescent cut) of the net $p_{0}$ of Figure 5 is depicted in Figure 6 (resp. Figure 7; Figure 8).

We are in fact interested in the simultaneous elimination of any number of reducible cuts, that we describe as follows. We write $p \rightrightarrows p^{\prime}$ if

$$
p=\left(c_{1}, \ldots, c_{k},\left\langle x_{1} \mid t_{1}\right\rangle, \ldots,\left\langle x_{n} \mid t_{n}\right\rangle,\left\langle\mu_{1} \mid \lambda_{1}\right\rangle, \ldots,\left\langle\mu_{l} \mid \lambda_{l}\right\rangle, \vec{c} ; \vec{t}\right)
$$

\footnotetext{
${ }^{4}$ Since the cuts of a net are given as a family rather than a sequence, the order in which we write cuts in this definition is not relevant: despite our abusive notation, the reduced cut need not be the first in the enumeration, because this enumeration is not fixed.

${ }^{5}$ We arbitrarily redirect the jumps to the first subtree to simplify the presentation, but we could equivalently have set $\jmath_{p^{\prime}}(\mu)$ to be any of the immediate subtrees of $\jmath_{p}(\mu)$, non deterministically: in fact, this slight generalization is necessary to deal with cut elimination in resource nets.

Other strategies for choosing the destination of a jump exist in the literature: for instance, one may be tempted to systematically redirect jumps to atoms, as it is done by Tortora de Falco [TdF00, Definition 1.3.3]. But this kind of transformation is not local and it would certainly complicate our arguments.
} 


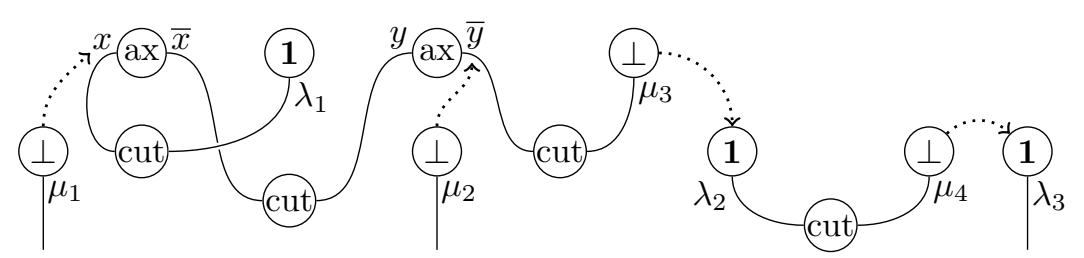

Figure 6: The net $p_{m}^{\prime}=\left(\left\langle x \mid \mathbf{1}_{\lambda_{1}}\right\rangle,\langle\bar{x} \mid y\rangle,\left\langle\bar{y} \mid \perp_{\mu_{3}}\right\rangle,\left\langle\mathbf{1}_{\lambda_{2}} \mid \perp_{\mu_{4}}\right\rangle ; \perp_{\mu_{1}}, \perp_{\mu_{2}}, \mathbf{1}_{\lambda_{3}}\right)$ with $\jmath_{p_{m}^{\prime}}: \mu_{1} \mapsto$ $x, \mu_{2} \mapsto \bar{y}, \mu_{3} \mapsto \lambda_{2}, \mu_{4} \mapsto \lambda_{3}$, so that $p_{0} \rightarrow_{m} p_{m}^{\prime}$.

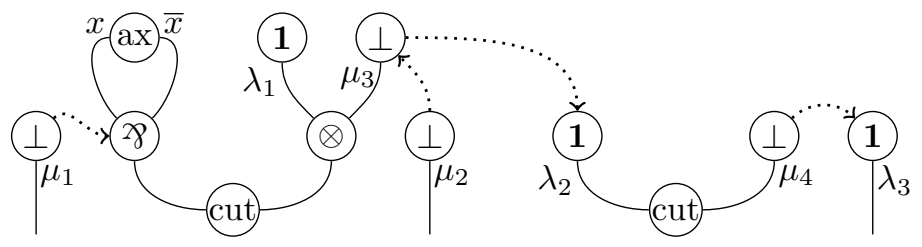

Figure 7: The net $p_{a}^{\prime}=\left(\langle\gamma(x, \bar{x})| \otimes\left(\mathbf{1}_{\lambda_{1}}, \perp_{\mu_{3}}\right\rangle,\left\langle\mathbf{1}_{\lambda_{2}} \mid \perp_{\mu_{4}}\right\rangle ; \perp_{\mu_{1}}, \perp_{\mu_{2}}, \mathbf{1}_{\lambda_{3}}\right)$ with $\jmath_{p_{a}^{\prime}}: \mu_{1} \mapsto$ 又 $(x, \bar{x}), \mu_{2} \mapsto \mu_{3}, \mu_{3} \mapsto \lambda_{2}, \mu_{4} \mapsto \lambda_{3}$, so that $p_{0} \rightarrow_{a} p_{a}^{\prime}$.

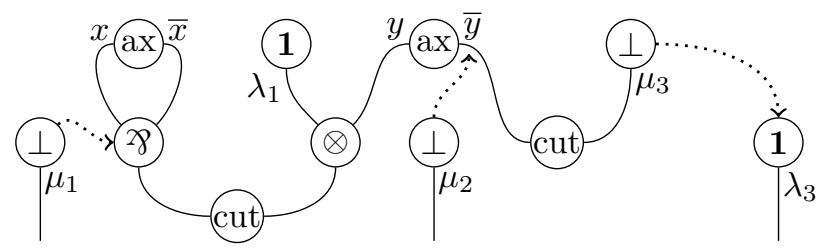

Figure 8: The net $p_{e}^{\prime}=\left(\left\langle\gamma(x, \bar{x}) \mid \otimes\left(\mathbf{1}_{\lambda_{1}}, y\right)\right\rangle,\left\langle\bar{y} \mid \perp_{\mu_{3}}\right\rangle ; \perp_{\mu_{1}}, \perp_{\mu_{2}}, \mathbf{1}_{\lambda_{3}}\right)$ with $\jmath_{p_{e}^{\prime}}: \mu_{1} \mapsto$ 子 $(x, \bar{x}), \mu_{2} \mapsto \bar{y}, \mu_{3} \mapsto \lambda_{3}$, so that $p_{0} \rightarrow_{e} p_{e}^{\prime}$.

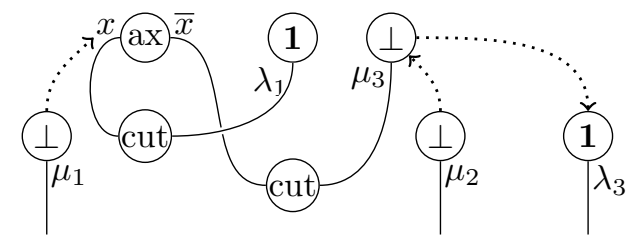

Figure 9: The net $p^{\prime}=\left(\left\langle x \mid \mathbf{1}_{\lambda_{1}}\right\rangle,\left\langle\bar{x} \mid \perp_{\mu_{3}}\right\rangle ; \perp_{\mu_{1}}, \perp_{\mu_{2}}, \mathbf{1}_{\lambda_{3}}\right)$ with $\jmath_{p^{\prime}}: \mu_{1} \mapsto x, \mu_{2} \mapsto \mu_{3}, \mu_{3} \mapsto \lambda_{3}$, so that $p_{0} \rightrightarrows p^{\prime}$.

and

assuming that:

$$
p^{\prime}=\left(\vec{c}_{1}^{\prime}, \ldots, \vec{c}_{k}^{\prime}, \vec{c} ; \vec{t}\right)\left[t_{1} / \bar{x}_{1}\right] \cdots\left[t_{n} / \bar{x}_{n}\right]
$$

- $c_{i} \rightarrow_{m} \vec{c}_{i}^{\prime}$ for $1 \leq i \leq k$,

- $\bar{x}_{i} \notin\left\{x_{1}, \ldots, x_{n}\right\}$ and $\bar{x}_{i} \notin \mathbf{V}\left(t_{j}\right)$ for $1 \leq i \leq j \leq n$, and

- $\jmath_{p}\left(\mu_{i}\right) \notin\left\{\mu_{j}, \lambda_{j}\right\}$ for $1 \leq i \leq j \leq l$.

It should be clear that $p^{\prime}$ is then obtained from $p$ by successively eliminating the particular cuts we have selected, thus performing $k$ steps of $\rightarrow_{m}, n$ steps of $\rightarrow_{a}, l$ steps of $\rightarrow_{e}$, in no particular order: indeed, one can check that any two elimination steps of distinct cuts 
commute on the nose. The resulting jump function $\jmath_{p^{\prime}}$ can be described directly, by inspecting the possible cases for $\jmath_{p}\left(\mu^{\prime}\right)$ with $\mu^{\prime} \in \mathbf{U}_{\perp}\left(p^{\prime}\right)$ :

- if $c_{i}=\left\langle\left.\otimes\left(u_{1}, \ldots, u_{r}\right)\right|^{\gamma 8}\left(v_{1}, \ldots, v_{r}\right)\right\rangle$ and, e.g., $\jmath_{p}\left(\mu^{\prime}\right)=\otimes\left(u_{1}, \ldots, u_{r}\right)$ then $\jmath_{p^{\prime}}\left(\mu^{\prime}\right)=$ $u_{1}\left[t_{1} / \bar{x}_{1}\right] \cdots\left[t_{n} / \bar{x}_{n}\right]$

- if $\jmath_{p}\left(\mu^{\prime}\right) \in\left\{x_{i}, \bar{x}_{i}\right\}$ then $\jmath_{p^{\prime}}\left(\mu^{\prime}\right)=t_{i}\left[t_{i+1} / \bar{x}_{i+1}\right] \cdots\left[t_{n} / \bar{x}_{n}\right]$;

- if $\jmath_{p}\left(\mu^{\prime}\right) \in\left\{\mu_{i}, \lambda_{i}\right\}$ then $\jmath_{p^{\prime}}\left(\mu^{\prime}\right)=\rho(i)\left[t_{1} / \bar{x}_{1}\right] \cdots\left[t_{n} / \bar{x}_{n}\right]$, where $\rho:\{1, \ldots, l\} \rightarrow \mathbf{T}(p)$ is the redirection function inductively defined by $\rho(j)=\rho(i)$ if $\jmath_{p}\left(\mu_{j}\right) \in\left\{\mu_{i}, \lambda_{i}\right\}$ (in which case $i<j)$ and $\rho(j)=\jmath_{p}\left(\mu_{j}\right)$ otherwise;

- otherwise $\jmath_{p^{\prime}}\left(\mu^{\prime}\right)=\jmath_{p}\left(\mu^{\prime}\right)\left[t_{1} / \bar{x}_{1}\right] \cdots\left[t_{n} / \bar{x}_{n}\right]$.

The result of simultaneously eliminating all the cuts of the net $p_{0}$ of Figure 5 is depicted in Figure 9.

This general description of parallel cut elimination is obviously not very handy. In order not to get lost in notation, we will restrict our attention to the particular case in which only cuts of the same nature are simultaneously eliminated: we write $p \rightrightarrows_{m} p^{\prime}$ if $n=l=0$ (multiplicative cuts only), $p \rightrightarrows_{a} p$ if $k=l=0$ (axiom cuts only), and $p \rightrightarrows_{e} p^{\prime}$ if $n=k=0$ (evanescent cuts only). Then we can decompose any parallel reduction $p \rightrightarrows p^{\prime}$ into three separate steps: e.g., $p \rightrightarrows_{m} \cdot \rightrightarrows_{a} \cdot \rightrightarrows_{e} p^{\prime}{ }^{6}$

2.3. Paths. In order to control the effect of parallel reduction on the size of proof nets, we rely on a side condition involving the length of switching paths, i.e. paths in the sense of Danos-Regnier's correctness criterion [DR89].

Let us write $\mathbf{T}_{\not \triangleright}(p)$ (resp. $\left.\mathbf{T}_{\otimes}(p)\right)$ for the set of the subtrees of $p$ of the form $\gamma\left(t_{1}, \ldots, t_{n}\right)$ (resp. $\left.\otimes\left(t_{1}, \ldots, t_{n}\right)\right)$. In our setting, a switching of a net $p$ is a map $I: \mathbf{T}_{\not}(p) \rightarrow \mathbf{T}(p)$ such that, for each $t=\not 2\left(t_{1}, \ldots t_{n}\right) \in \mathbf{T}_{\ngtr}(p), I(t) \in\left\{t_{1}, \ldots, t_{n}\right\}$. Given a net $p$ and a switching $I$ of $p$, the associated switching graph is the unoriented graph with vertices in $\mathbf{T}(p)$ and edges given as follows:

- one axiom edge $\sim_{\{x, \bar{x}\}}$ for each axiom $\{x, \bar{x}\} \subseteq \mathbf{V}(p)$, connecting $x$ and $\bar{x}$;

- one $\otimes$-edge $\sim_{t, t_{i}}$ for each pair $\left(t, t_{i}\right)$ with $t=\otimes\left(t_{1}, \ldots, t_{n}\right) \in \mathbf{T}_{\otimes}(p)$, connecting $t$ and $t_{i}$;

- one 8 -edge $\sim_{t}$ for each $t \in \mathbf{T}_{8}(p)$, connecting $t$ and $I(t)$;

- one jump edge $\sim_{\mu}$ for each $\mu \in \mathbf{U}_{\perp}(p)$, connecting $\mu$ and $\jmath_{p}(\mu)$;

- one cut edge $\sim_{c}$ for each cut $c=\langle t \mid s\rangle \in \mathbf{C}(p)$, connecting $t$ and $s$.

Whenever necessary, we may write, e.g., $\sim_{e}^{p}$ or $\sim_{e}^{p, I}$ for the edge $\sim_{e}$ to make the underlying net and switching explicit. On the other hand, we will often simply write $e$ instead of $\sim_{e}$ for denoting an edge. Each edge $e$ induces a symmetric relation, involving at most two subtrees of $p$ : we write $t \sim_{e} u$, and say $t$ and $u$ are adjacent whenever $t$ and $u$ are connected by $e$. A priori, it might be the case that distinct edges induce the same relation: for instance, if $c=\langle x \mid \bar{x}\rangle \in \mathbf{C}(p)$, we have $x \sim_{c} \bar{x}$ as well as $x \sim_{\{x, \bar{x}\}} \bar{x}$; and if $\jmath_{p}(\mu)=\mu^{\prime}$ and $\jmath_{p}\left(\mu^{\prime}\right)=\mu$, we have $\mu \sim_{\mu} \mu^{\prime}$ as well as $\mu \sim_{\mu^{\prime}} \mu^{\prime}$. Avoiding such cycles is precisely the purpose of the correctness criterion.

Given a switching $I$ in $p$, an $I$-path is the data of a tree $t_{0} \in \mathbf{T}(p)$ and of a sequence $\left(e_{1}, \ldots, e_{n}\right)$ of pairwise distinct consecutive edges starting from $t_{0}$ : in other words, we require that there exist $t_{1}, \ldots, t_{n} \in \mathbf{T}(p)$ such that, for each $i \in\{1, \ldots, n\}, t_{i-1} \sim_{e_{i}} t_{i}{ }^{7}$

\footnotetext{
${ }^{6}$ Of course, the converse does not hold: for instance the reductions $(\langle\not \gamma(x, \bar{x}) \mid \otimes(y, z)\rangle ; \bar{y}, \bar{z}) \rightrightarrows_{m}$ $(\langle x \mid y\rangle,\langle\bar{x} \mid z\rangle ; \bar{y}, \bar{z}) \rightrightarrows a(\langle y \mid z\rangle ; \bar{y}, \bar{z})$ cannot be performed in a single step, as the cut $\langle x \mid y\rangle$ was newly created.

${ }^{7}$ In standard terminology of graph theory, an $I$-path in $p$ is a trail in the switching graph induced by $p$ and $I$.
} 


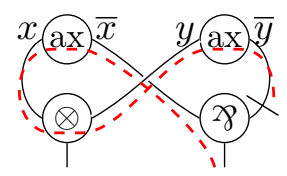

Figure 10: A path in $(; \otimes(x, y), \not \supset(\bar{y}, \bar{x}))$ for switching $I: \not 8(\bar{y}, \bar{x}) \mapsto \bar{x}$ (we strike out the other premise).

For instance, if $p=(; \otimes(x, y), \not \supset(\bar{y}, \bar{x}))$ and $I(\not \supset(\bar{y}, \bar{x}))=\bar{x}$, then the chain of adjacencies $\ngtr(\bar{x}, \bar{y}) \sim_{\gamma(\bar{x}, \bar{y})} \bar{x} \sim_{\{x, \bar{x}\}} x \sim_{\otimes(x, y), x} \otimes(x, y) \sim_{\otimes(x, y), y} y \sim_{\{y, \bar{y}\}} \bar{y}$ defines a maximal $I$-path in $p$ (see Figure 10).

We write $\mathbf{P}(p, I)$ for the set of all $I$-paths in $p$. We write $\chi: t_{0} \rightsquigarrow_{p, I} t_{n}$ whenever $\chi=t_{0} \sim_{e_{1}} \cdots \sim_{e_{n}} t_{n}$ is an $I$-path from $t_{0}$ to $t_{n}$ in $p$ : with these notations, we say $\chi$ visits the trees $t_{0}, \ldots, t_{n}$, and $\chi$ crosses the edges $e_{1}, \ldots, e_{n}$; moreover we write $\ln (\chi)=n$ for the length of $\chi$. A subpath of $\chi$ is any $I$-path of the form $t_{i} \sim_{e_{i+1}} \cdots \sim_{e_{i+k}} t_{i+k}$. We write $\chi^{\dagger}$ for the reverse $I$-path: $\chi^{\dagger}=t_{n} \sim_{e_{n}} \cdots \sim_{e_{0}} t_{0}$. The empty path from $t \in \mathbf{T}(p)$ is the only $\epsilon_{t}: t \rightsquigarrow_{p, I} t$ of length 0 . We say $I$-paths $\chi$ and $\chi^{\prime}$ are disjoint if no edge is crossed by both $\chi$ and $\chi^{\prime}$. Observe that disjoint paths can visit common trees: in particular, if $\chi: t \rightsquigarrow p, I s$ and $\chi^{\prime}: s \rightsquigarrow_{p, I} u$ are disjoint, we write $\chi \chi^{\prime}: t \rightsquigarrow p, I u$ for the concatenation of $\chi$ and $\chi^{\prime}$.

We call path in $p$ any $I$-path for $I$ a switching of $p$, we write $\mathbf{P}(p)$ for the set of all paths in $p$, and we denote by $\ln (p)=\max \{\ln (\chi) \mid \chi \in \mathbf{P}(p)\}$ the maximal length of a path in $p$. We then write $\chi: t \rightsquigarrow_{p} s$ if $\chi \in \mathbf{P}(p)$ is a path from $t$ to $s$ in $p$, and we write $t \rightsquigarrow p s$, or simply $t \rightsquigarrow s$, whenever such a path exists. This relation on $\mathbf{T}(p)$ is reflexive (via the empty path $\epsilon_{t}: t \rightsquigarrow t$ ) and symmetric (via reversing paths). Observe that if $\chi_{1}, \cdots, \chi_{n} \in \mathbf{P}(p)$ are pairwise disjoint, then there exists $I$ such that $\chi_{i} \in \mathbf{P}(p, I)$ for $1 \leq i \leq n$. This does not make the relation $\rightsquigarrow p$ transitive: for instance, if $p=(; x, \not \supset(\bar{x}, \bar{y}), y)$, we have paths

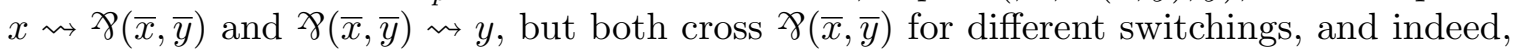
there is no path $x \rightsquigarrow y$.

We say a net $p$ is acyclic if, for all $\chi \in \mathbf{P}(p)$ and $t \in \mathbf{T}(p)$, $\chi$ visits $t$ at most once: in other words, there is no (non-empty) cycle $\chi: t \rightsquigarrow t$. Notice that, given any family of pairwise distinct cuts in an acyclic net $p$, it is always possible to satisfy the side conditions on free variables and on jumps necessary to reduce these cuts in parallel (provided each cut in the family has the shape of a multiplicative, axiom or evanescent cut). Moreover, it is a very standard result that acyclicity is preserved by cut elimination:

Lemma 2.3. If $p^{\prime}$ is obtained from $p$ by cut elimination and $p$ is acyclic then so is $p^{\prime}$.

Proof. It suffices to check that if $p \rightarrow p^{\prime}$ then any cycle in $p^{\prime}$ induces a cycle in $p{ }^{8}$

From now on, we consider acyclic nets only.

\section{Bounding THE SIZE OF ANTIREDUCTS: THREE KINDS OF CUTS}

In this section, we show that the loss of size during a parallel reduction $p \rightrightarrows_{m} q, p \rightrightarrows_{a} q$ or $p \rightrightarrows_{e} q$ is directly controlled by $\ln (p), \mathbf{j d}(p)$ and $\operatorname{size}(q)$ : more precisely, we show that the ratio $\frac{\operatorname{size}(p)}{\operatorname{size}(q)}$ is bounded by a function of $\ln (p)$ and $\mathbf{j d}(p)$ in each case.

\footnotetext{
${ }^{8}$ We do not detail the proof as it is quite standard. We will moreover generalize this technique to all paths (and not only cycles) in the next section.
} 
3.1. Elimination of multiplicative cuts. The elimination of multiplicative cuts cannot decrease the size by more than a half:

Lemma 3.1. If $p \rightrightarrows_{m} q$ then $\operatorname{size}(p) \leq 2 \operatorname{size}(q)$.

Proof. Since the elimination of a multiplicative cut does not affect the rest of the (bare) net, it is sufficient to observe that if $c \rightarrow_{m} \vec{c}$ then $\operatorname{size}(c)=2+\operatorname{size}(\vec{c}) \leq 2 \operatorname{size}(\vec{c}) .{ }^{9}$

So in this case, $\ln (p)$ and $\mathbf{j} \mathbf{d}(p)$ actually play no rôle.

3.2. Elimination of axiom cuts. Observe that:

- if $x \in \mathbf{V}(\gamma)$ then $\operatorname{size}(\gamma[t / x])=\operatorname{size}(\gamma)+\operatorname{size}(t)-1$;

- if $x \notin \mathbf{V}(\gamma)$ then $\operatorname{size}(\gamma[t / x])=\operatorname{size}(\gamma)$.

It follows that, in the elimination of a single axiom cut $p \rightarrow_{a} q$, we have $\operatorname{size}(p)=\operatorname{size}(q)+2$. But we cannot reproduce the proof of Lemma 3.1 for $\rightrightarrows_{a}$ : as depicted in Figure 2, a chain of arbitrarily many axiom cuts may reduce into a single wire. We can bound the length of those chains by $\ln (p)$, however, and this allows us to bound the loss of size during reduction.

Lemma 3.2. If $p \rightrightarrows_{a} q$ then $\operatorname{size}(p) \leq(\ln (p)+1) \operatorname{size}(q)$.

Proof. Assume $p=\left(\left\langle x_{1} \mid t_{1}\right\rangle, \ldots,\left\langle x_{n} \mid t_{n}\right\rangle, \vec{c} ; \vec{s}\right)$ and $q=(\vec{c} ; \vec{s})\left[t_{1} / \bar{x}_{1}\right] \cdots\left[t_{n} / \bar{x}_{n}\right]$ with $\bar{x}_{i} \notin$ $\left\{x_{1}, \ldots, x_{n}\right\}$ and $\bar{x}_{i} \notin \mathbf{V}\left(t_{j}\right)$ for $1 \leq i \leq j \leq n$. To establish the result in this case, we make the chains of eliminated axiom cuts explicit.

Due to the condition on free variables, we can partition $\left\langle x_{1} \mid t_{1}\right\rangle, \ldots,\left\langle x_{n} \mid t_{n}\right\rangle$ into tuples $\vec{c}_{1}, \ldots, \vec{c}_{k}$ of the shape $\vec{c}_{i}=\left(\left\langle x_{0}^{i} \mid \bar{x}_{1}^{i}\right\rangle, \ldots,\left\langle x_{n_{i}-1}^{i} \mid \bar{x}_{n_{i}}^{i}\right\rangle,\left\langle x_{n_{i}}^{i} \mid t^{i}\right\rangle\right)$ so that:

- $x_{j}^{i} \in\left\{x_{1}, \ldots, x_{n}\right\}$ for $1 \leq i \leq k$ and $0 \leq j \leq n_{i}$;

- $\bar{x}_{j}^{i} \in\left\{t_{1}, \ldots, t_{n}\right\}$ for $1 \leq i \leq k$ and $1 \leq j \leq n_{i}$;

- $t^{i} \in\left\{t_{1}, \ldots, t_{n}\right\}$ for $1 \leq i \leq k$;

- each $\vec{c}_{i}$ is maximal with this shape, i.e. $\bar{x}_{0}^{i} \notin\left\{t_{1}, \ldots, t_{n}\right\}$ and $t^{i} \notin\left\{\bar{x}_{1}, \ldots, \bar{x}_{n}\right\}$.

Without loss of generality, we can moreover require that, if $i<i^{\prime}$, then $\left\langle x_{n_{i}}^{i} \mid t^{i}\right\rangle$ occurs before $\left\langle x_{n_{i^{\prime}}}^{i^{\prime}} \mid t^{i^{\prime}}\right\rangle$ in the tuple $\left(\left\langle x_{1} \mid t_{1}\right\rangle, \ldots,\left\langle x_{n} \mid t_{n}\right\rangle\right)$. Moreover observe that, by the condition on free variables, the order of the cuts in each $\vec{c}_{i}$ is necessarily the same as in $\left(\left\langle x_{1} \mid t_{1}\right\rangle, \ldots,\left\langle x_{n} \mid t_{n}\right\rangle\right)$.

By a standard result on substitutions, if $x \neq y, x \notin \mathbf{V}(v)$ and $y \notin \mathbf{V}(u)$ then $\gamma[u / x][v / y]=\gamma[v / y][u / x]$ :

- if $i \neq i^{\prime}$, we have $\bar{x}_{j}^{i} \neq \bar{x}_{j^{\prime}}^{i^{\prime}}$ for $0 \leq j \leq n_{i}$ and $0 \leq j^{\prime} \leq n_{i^{\prime}}$, so the substitutions $\left[\bar{x}_{j+1}^{i} / \bar{x}_{j}^{i}\right]$ and $\left[\bar{x}_{j^{\prime}+1}^{i^{\prime}} / \bar{x}_{j^{\prime}}^{i^{\prime}}\right]$ always commute for $1 \leq i<i^{\prime} \leq k, 0 \leq j<n_{i}$ and $0 \leq j^{\prime}<n_{j^{\prime}}$;

- if $i<i^{\prime}$ and $\left\langle x_{j^{\prime}}^{i^{\prime}} \mid \bar{x}_{j^{\prime}+1}^{i^{\prime}}\right\rangle$ occurs before $\left\langle x_{n_{i}}^{i} \mid t^{i}\right\rangle$ in $\left(\left\langle x_{1} \mid t_{1}\right\rangle, \ldots,\left\langle x_{n} \mid t_{n}\right\rangle\right)$, the condition on free variables imposes that $\bar{x}_{j^{\prime}}^{i^{\prime}} \notin \mathbf{V}\left(t^{i}\right)$ so the substitutions $\left[t^{i} / \bar{x}_{n_{i}}^{i}\right]$ and $\left[\bar{x}_{j^{\prime}+1}^{i^{\prime}} / \bar{x}_{j^{\prime}}^{i^{\prime}}\right]$ commute in this case.

By iterating those two observations, we can reorder the substitutions in $q$ and obtain:

$$
\begin{aligned}
q & =(\vec{c} ; \vec{s})\left[t_{1} / \bar{x}_{1}\right] \cdots\left[t_{n} / \bar{x}_{n}\right] \\
& =(\vec{c} ; \vec{s})\left[\bar{x}_{1}^{1} / \bar{x}_{0}^{1}\right] \cdots\left[\bar{x}_{n_{1}}^{1} / \bar{x}_{n_{1}-1}^{1}\right]\left[t^{1} / \bar{x}_{n_{1}}^{1}\right] \cdots\left[\bar{x}_{1}^{k} / \bar{x}_{0}^{k}\right] \cdots\left[\bar{x}_{n_{k}}^{k} / \bar{x}_{n_{k}-1}^{k}\right]\left[t^{k} / \bar{x}_{n_{k}}^{k}\right] \\
& =(\vec{c} ; \vec{s})\left[t^{1} / \bar{x}_{0}^{1}\right] \cdots\left[t^{k} / \bar{x}_{0}^{k}\right] .
\end{aligned}
$$

\footnotetext{
${ }^{9}$ This is due to the fact that we distinguish between strict connectives and their nullary versions, that are subject to evanescent reductions.
} 
It follows that $\operatorname{size}(q)=\operatorname{size}(\vec{c})+\operatorname{size}(\vec{s})+\sum_{i=1}^{k} \operatorname{size}\left(t^{i}\right)-k$. For $1 \leq i \leq k, \vec{c}_{i}$ induces a path $\bar{x}_{i}^{0} \rightsquigarrow t^{i}$ of length $2 n_{i}+2\left(n_{i}+1\right.$ cuts and $n_{i}+1$ axioms). Hence $2 n_{i} \leq \ln (p)-2$ and:

$$
\begin{aligned}
\operatorname{size}(p) & =\operatorname{size}(\vec{c})+\operatorname{size}(\vec{s})+\sum_{i=1}^{k}\left(\operatorname{size}\left(t^{i}\right)+2 n_{i}+1\right) \\
& \leq \operatorname{size}(\vec{c})+\operatorname{size}(\vec{s})+\sum_{i=1}^{k} \operatorname{size}\left(t^{i}\right)+k(\ln (p)-1) \\
& \leq \operatorname{size}(q)+k \ln (p) .
\end{aligned}
$$

To conclude, it will be sufficient to prove that $\operatorname{size}(q) \geq k$. For $1 \leq i \leq k$, let $A_{i}=\left\{j>i \mid \bar{x}_{0}^{j} \in \mathbf{V}\left(t^{i}\right)\right\}$, and then let $A_{0}=\left\{i \mid \bar{x}_{0}^{i} \in \mathbf{V}(\vec{c}, \vec{s})\right\}$. It follows from the construction that $\left\{A_{0}, \ldots, A_{k-1}\right\}$ is a partition (possibly including empty sets) of $\{1, \ldots, k\}$. By construction, for each $j \in A_{i}, \bar{x}_{0}^{j}$ is a strict subtree of $t^{i}$ : it follows that $\operatorname{size}\left(t^{i}\right)>\# A_{i}$. Now consider $q_{i}=(\vec{c} ; \vec{s})\left[t^{1} / \bar{x}_{0}^{1}\right] \cdots\left[t^{i} / \bar{x}_{0}^{i}\right]$ for $0 \leq i \leq k$ so that $q=q_{k}$. For $1 \leq i \leq k$, we obtain $\operatorname{size}\left(q_{i}\right)=\operatorname{size}\left(q_{i-1}\right)+\operatorname{size}\left(t^{i}\right)-1 \geq \operatorname{size}\left(q_{i-1}\right)+\# A_{i}$. Also observe that $\operatorname{size}\left(q_{0}\right)=$ $\operatorname{size}(\vec{c} ; \vec{s}) \geq \# A_{0}$. Then we can conclude: $\operatorname{size}(q)=\operatorname{size}\left(q_{k}\right) \geq \sum_{i=0}^{k} \# A_{i}=k$.

3.3. Elimination of evanescent cuts. We now consider the case of a reduction $p \rightrightarrows_{e} q$ : we bound the maximal number of evanescent cuts appearing in $p$ by a function of $\ln (p)$, $\mathbf{j d}(p)$ and $\operatorname{size}(q)$.

We rely on the basic fact that if $t \in \mathbf{T}(q) \subseteq \mathbf{T}(p)$, then there are at most $\mathbf{j} \mathbf{d}(p)$ evanescent cuts of $p$ that jump to $t$. The main difficulty is that an evanescent cut of $p$ can jump to another evanescent cut of $p$, that is also eliminated in the step $p \rightrightarrows_{e} q$. See Figure 11 for a graphical representation of the critical case. To deal with this phenomenon, we observe that a sequence of cuts $\left\langle\mu_{1} \mid \lambda_{1}\right\rangle, \ldots,\left\langle\mu_{n} \mid \lambda_{n}\right\rangle$ with $\jmath_{p}\left(\mu_{i}\right) \in\left\{\lambda_{i+1}, \mu_{i+1}\right\}$ for all $i \in\{1, \ldots, n-1\}$, induces a path of length at least $n$ : hence $n \leq \ln (p)$.

Definition 3.3. We define for all $n \in \mathbf{N}$ and all $t \in \mathbf{T}(p), \mathbf{I}^{n}(t)$ as follows : $\mathbf{I}^{0}(t)=\jmath_{p}^{-1}(t)=$ $\left\{\mu \in \mathbf{U}_{\perp}(p) \mid \jmath_{p}(\mu)=t\right\}$, and $\mathbf{I}^{m+1}(t)=\left\{\mu^{\prime} \in \mathbf{U}_{\perp}(p) \mid \jmath_{p}\left(\mu^{\prime}\right) \in\{\mu, \lambda\},\langle\mu \mid \lambda\rangle \in \mathbf{C}(p), \mu \in\right.$ $\left.\mathbf{I}^{m}(t)\right\}$.

We can already observe that $\# \mathbf{I}^{0}(t)=\mathbf{j d}(t)$. This definition is parametrized by $\jmath_{p}$, and may we write $\mathbf{I}_{p}^{n}(t)$ to make the underlying net explicit.

Lemma 3.4. Let $p, q$ be two nets such that $p \rightrightarrows_{e} q$. Then:

(1) for all $t \in \mathbf{T}(p)$, \# $\left(\bigcup_{i \in \mathbf{N}} \mathbf{I}_{p}^{i}(t)\right) \leq(2 \mathbf{j d}(p))^{\mathbf{l n}(p)+1}$;

(2) there are at most $\operatorname{size}(q) \times(2 \mathbf{j d}(p))^{\mathbf{l n}(p)+1}$ evanescent cuts in $\mathbf{C}(p)$.

Proof. We first establish that the set $\left\{n \in \mathbf{N} \mid \mathbf{I}^{n}(t) \neq \emptyset\right\}$ is finite for all $t$. Indeed, for each $\mu_{n} \in \mathbf{I}^{n}(t)$, there is a sequence of cuts $c_{0}, \ldots, c_{n-1}$ such that, writing $c_{i}=\left\langle\lambda_{i} \mid \mu_{i}\right\rangle$, the unique path from $\mu_{n}$ to $t$ is $\chi=\chi_{n} \cdots \chi_{1}\left(\mu_{0} \sim_{\mu_{0}} t\right)$ where, for $1 \leq i \leq n$ :

- either $\jmath_{p}\left(\mu_{i}\right)=\lambda_{i-1}$ and $\chi_{i}=\mu_{i} \sim_{\mu_{i}} \lambda_{i-1} \sim_{c_{i-1}} \mu_{i-1}$;

- or $\jmath_{p}\left(\mu_{i}\right)=\mu_{i-1}$ and $\chi_{i}=\mu_{i} \sim_{\mu_{i}} \mu_{i-1}$.

We observe that $\ln (\chi) \geq n+1$, and then we deduce $n<\ln (p)$ as soon as $\mathbf{I}^{n}(t) \neq \emptyset$.

Now we bound the size of each $\mathbf{I}_{p}^{n}(t)$ : we show that $\# \mathbf{I}_{p}^{n}(t) \leq(2 \mathbf{j d}(p))^{n+1}$, by induction on $n$. We already have $\# \mathbf{I}_{p}^{0}(t)=\mathbf{j} \mathbf{d}(t) \leq \mathbf{j d}(p)$. Now assume the result holds for $n \geq 0$. 


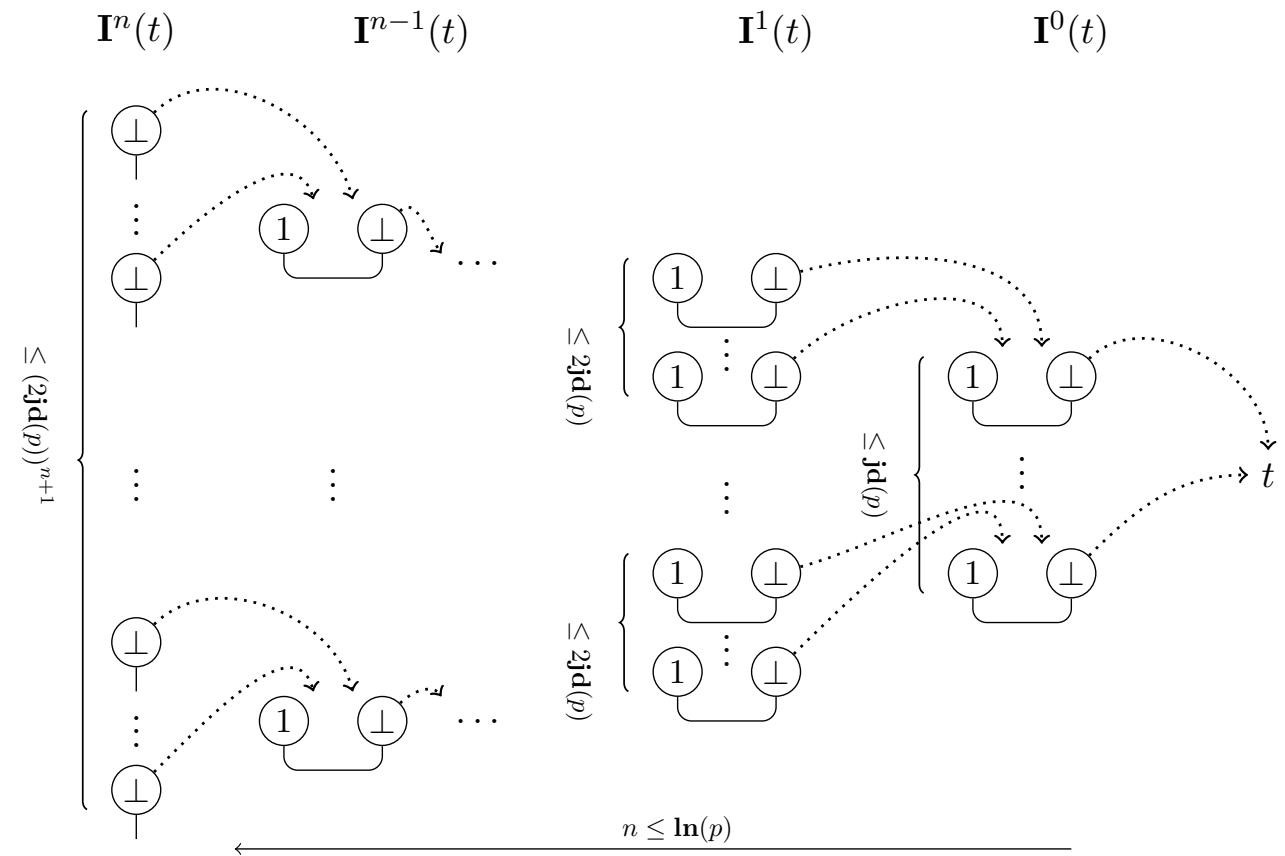

Figure 11: Evanescent reductions : critical case

Then, for each $c=\langle\mu \mid \lambda\rangle \in \mathbf{C}(p)$ such that $\mu \in \mathbf{I}_{p}^{n}(t)$, the number of $\mu^{\prime}$ such that $\jmath_{p}\left(\mu^{\prime}\right) \in c$ is at most $\mathbf{j d}(\mu)+\mathbf{j d}(\lambda) \leq 2 \mathbf{j d}(p)$. We obtain: $\# \mathbf{I}_{p}^{n+1}(t) \leq 2 \mathbf{j d}(p) \# \mathbf{I}_{p}^{n}$, which enables the induction.

We thus get \# $\left(\bigcup_{i \in \mathbf{N}} \mathbf{I}_{p}^{i}(t)\right) \leq \sum_{i=0}^{\ln (p)-1}(2 \mathbf{j d}(p))^{i+1} \leq(2 \mathbf{j d}(p))^{\ln (p)+1}$ which entails (1). To deduce (2) from (1), it will be sufficient to show that, for each $\mu \in \mathbf{U}_{\perp}(p)$, there exists $t \in \mathbf{T}(q)$ such that $\mu \in \mathbf{I}_{p}^{k}(t)$ for some $k \in \mathbf{N}$ : indeed the number of evanescent cuts in $p$ is obviously bounded by $\# \mathbf{U}_{\perp}(p)$.

For that purpose, write $\mu_{0}=\mu$ and let $\left(c_{i}\right)_{i \in\{1, \ldots, k\}}$ be the longest sequence of cuts $c_{i}=\left\langle\lambda_{i} \mid \mu_{i}\right\rangle \in \mathbf{C}(p)$ such that, for all $i \in\{0, \ldots, k-1\}, \jmath_{p}\left(\mu_{i}\right) \in\left\{\mu_{i+1}, \lambda_{i+1}\right\}$ : such a maximal sequence exists by acyclicity. Necessarily, $\jmath_{p}\left(\mu_{k}\right)$ is not part of an evanescent cut in $\mathbf{C}(p)$, so $\jmath_{p}\left(\mu_{k}\right) \in \mathbf{T}(q)$ : we conclude since $\mu_{0} \in \mathbf{I}_{p}^{k}\left(\jmath_{p}\left(\mu_{k}\right)\right)$.

Writing $\psi(i, j, k)=i\left(1+2(2 j)^{k+1}\right)$, we obtain:

Lemma 3.5. If $p \rightrightarrows_{e} q$, then $\operatorname{size}(p) \leq \psi(\operatorname{size}(q), \mathbf{j d}(p), \ln (p))$.

Proof. Writing $p=\left(\left\langle\mu_{1} \mid \lambda_{1}\right\rangle, \ldots,\left\langle\mu_{n} \mid \lambda_{n}\right\rangle, \vec{c} ; \vec{t}\right)$ and $q=(\vec{c} ; \vec{t})$, we obtain

$$
\operatorname{size}(p)=\operatorname{size}(q)+2 n \leq \operatorname{size}(q)\left(1+2(2 \mathbf{j d}(p))^{\ln (p)+1}\right)
$$

by Lemma 3.4.

3.4. Towards the general case. Recall that any parallel cut elimination step $p \rightrightarrows q$ can be decomposed into, e.g.: $p \rightrightarrows_{e} p^{\prime} \rightrightarrows_{m} p^{\prime \prime} \rightrightarrows_{a} q$. We would like to apply the previous results to this sequence of reductions, in order to bound the size of $p$ by a function of $\operatorname{size}(q), \ln (p)$ and $\mathbf{j d}(p)$. Observe however that this would require us to infer a bound on $\ln \left(p^{\prime \prime}\right)$ from the bounds on $p$, in order to apply Lemma 3.2 . 

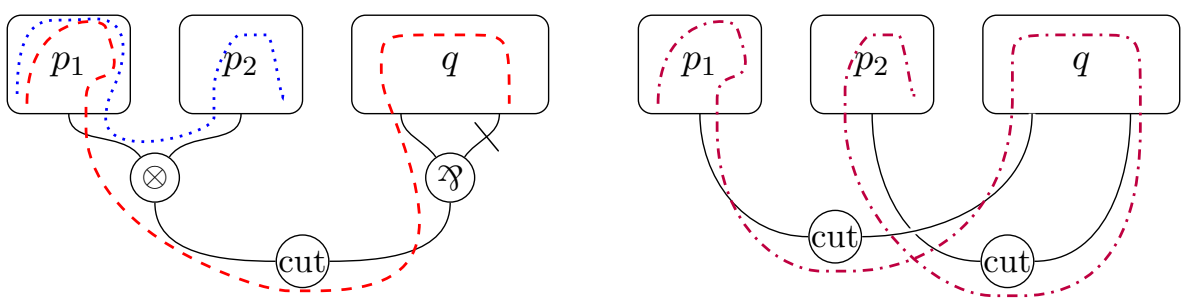

Figure 12: A cut, the resulting slipknot, and examples of paths before and after reduction

More generally, to be able to apply our results to a sequence of reductions $p \rightrightarrows \cdots \rightrightarrows q$, we need to ensure that for any reduction $p \rightrightarrows p^{\prime}$, we can bound $\ln \left(p^{\prime}\right)$ and $\mathbf{j d}\left(p^{\prime}\right)$ by functions of $\ln (p)$ and $\mathbf{j d}(p)$. This is the subject of the following two sections.

\section{VARIATIONS OF $\ln (p)$ UNDER REDUCTION}

Here we establish that the possible increase of $\ln (p)$ under reduction is bounded. It should be clear that:

Lemma 4.1. If $p \rightrightarrows_{a} q$ or $p \rightrightarrows_{e} q$, then $\ln (q) \leq \ln (p)$.

Indeed axiom and evanescent reductions only shorten paths, without really changing the topology of the net.

In the case of multiplicative cuts however, cuts are duplicated and new paths are created. Consider for instance a net $r$, as in Figure 12, obtained from three nets $p_{1}, p_{2}$ and $q$, by forming the cut $\left\langle\otimes\left(t_{1}, t_{2}\right) \mid \not \gamma\left(s_{1}, s_{2}\right)\right\rangle$ where $t_{1} \in \mathbf{T}\left(p_{1}\right), t_{2} \in \mathbf{T}\left(p_{2}\right)$ and $s_{1}, s_{2} \in \mathbf{T}(q)$. Observe that, in the reduct $r^{\prime}$ obtained by forming two cuts $\left\langle t_{1} \mid s_{1}\right\rangle$ and $\left\langle t_{2} \mid s_{2}\right\rangle$, we may very well form a path that travels from $p_{1}$ to $q$ then $p_{2}$; while in $p$, this is forbidden by any switching of $\not\left(s_{1}, s_{2}\right)$. For instance, if we consider $I\left(\not \partial\left(s_{1}, s_{2}\right)\right)=s_{1}$, we may only form a path between $p_{1}$ and $p_{2}$ through $\otimes\left(t_{1}, t_{2}\right)$, or a path between $q$ and one of the $p_{i}$ 's, through $s_{1}$ and the cut.

In the remainder of this section, we fix a reduction step $p \rightrightarrows_{m} q$, and we show that the previous example describes a general mechanism: a path $\chi$ in $q$ that is not already in $p$ must involve a subpath $\chi^{\prime}$ between two residuals of a cut of $p$ that was eliminated in $p \rightrightarrows_{m} q$. We refer to this situation as a slipknot in $\chi$.

More formally, consider $c=\left\langle t_{0} \mid s_{0}\right\rangle \in \mathbf{C}(p)$ with $t_{0}=\otimes\left(t_{1}, \ldots, t_{n}\right)$ and $s_{0}=\not \gamma\left(s_{1}, \ldots, s_{n}\right)$ and assume $c$ is eliminated in the reduction $p \rightrightarrows_{m} q$ : then the residuals of $c$ in $q$ are the cuts $\left\langle t_{i} \mid s_{i}\right\rangle \in \mathbf{C}(q)$ for $1 \leq i \leq n$. For any edge $e$, we write $(e)$ for any length 1 path $t \sim_{e} s$. If $\chi \in \mathbf{P}(q)$, a slipknot of $\chi$ is any subpath $(d) \xi\left(d^{\prime}\right)$ where $d$ and $d^{\prime}$ are (necessarily distinct) residuals of the same cut in $p$. In the remaining of this section, we show that a path in $q$ is necessarily obtained by alternating paths (essentially) in $p$ and slipknots in $q$, that recursively consist of such alternations. This will allow us to bound $\ln (q)$ depending on $\ln (p)$, by reasoning inductively on these paths.

4.1. Preserved paths. Notice that $\mathbf{T}(q) \subseteq \mathbf{T}(p)$ and, given a switching $J$ of $q$, it is always possible to extend $J$ into a switching $I$ of $p$ : to determine $I$ uniquely amounts to select a premise for each 2 -tree in an eliminated cut.

Let $J$ be a switching of $q$ and $I$ an extension of $J$ on $p$. Observe that if $t \sim_{e}^{p, I} t^{\prime}$ and neither $t$ nor $t^{\prime}$ is an element of an eliminated cut, then $e$ is also an edge of $q$ that is not 
a residual cut; conversely, if $t \sim_{e}^{q, J} t^{\prime}$ and $e$ is not a residual cut, then $e$ is also an edge of $p$. We then say the edge $e$ is preserved by the reduction $p \rightrightarrows_{m} q$. If a preserved edge $e$ is a cut, an axiom, a $\otimes$-edge or a 2 -edge, then $e$ has the same endpoints in $p$ and in $q: t \sim_{e}^{p, I} t^{\prime}$ iff $t \sim_{e}^{q, J} t^{\prime}$. If $e=\mu$ is a jump, one endpoint might be changed: indeed, $\mu \sim_{e}^{p} \jmath_{p}(\mu)$ and $\mu \sim \sim_{e}^{q} \jmath_{q}(\mu)$, and we might have $\jmath_{p}(\mu) \neq \jmath_{q}(\mu)$ when $\jmath_{p}(\mu)$ is part of an eliminated cut. In this case, we say $e$ is a redirected jump. We say $u \in \mathbf{T}(p)$ is an anchor of $v \in \mathbf{T}(q)$, if either $u=v$ or $u$ is involved in an eliminated cut $c=\left\langle u \mid u^{\prime}\right\rangle$, and either $u=\otimes(\vec{t})$ and $v \in \vec{t}$ or $u=\not(\vec{s})$ and $v \in \vec{s}$.

Lemma 4.2. Assume $(d) \chi\left(d^{\prime}\right) \in \mathbf{P}(q)$ and $d$ and $d^{\prime}$ are residuals. Then $\chi$ is non empty, and its first and last edges are preserved.

Proof. This is a direct consequence of the fact that if $(c)(e)$ is a path and $c$ is a cut then $e$ is not a cut.

Observe that even if a path $\chi \in \mathbf{P}(q)$ crosses preserved edges only, it is not sufficient to have $\chi \in \mathbf{P}(p)$, because the endpoints of redirected jumps might change. We say $\chi$ is a preserved path if $\chi$ crosses preserved edges only, and we can write either $\chi=\chi^{\prime}$ or $\chi=\left(t \sim_{\mu} \perp_{\mu}\right) \chi^{\prime}$ or $\chi^{\prime}\left(\perp_{\mu^{\prime}} \sim_{\mu^{\prime}} t^{\prime}\right)$ or $\chi=\left(t \sim_{\mu} \perp_{\mu}\right) \chi^{\prime}\left(\perp_{\mu^{\prime}} \sim_{\mu^{\prime}} t^{\prime}\right)$ where $\chi^{\prime}$ crosses no redirected jump.

Lemma 4.3. Any non empty preserved path $\chi: t \rightsquigarrow_{q} t^{\prime}$ induces a unique preserved path $\chi^{-}: s \rightsquigarrow p s^{\prime}$ with the same sequence of edges: in particular, $\chi^{-} \in \mathbf{P}(p, I)$ as soon as $\chi \in \mathbf{P}(q, J)$ and $I$ is an extension of $J$; and $s$ (resp. $\left.s^{\prime}\right)$ is an anchor of $t$ (resp. $\left.t^{\prime}{ }^{\prime}\right)$. Moreover, if $\chi_{1} \chi_{2}$ is a preserved path then $\left(\chi_{1} \chi_{2}\right)^{-}=\chi_{1}^{-} \chi_{2}^{-} \in \mathbf{P}(p)$.

Proof. The first part is a direct consequence of the definition. If moreover $\chi_{1} \chi_{2}$ is a preserved path, and neither $\chi_{1}$ nor $\chi_{2}$ is an empty path, then neither the last edge of $\chi_{1}$ nor the first edge of $\chi_{2}$ can be a redirected jump.

By convention, if $\chi$ is empty, we set $\chi^{-}=\chi$. We say $\chi^{-}$is the path of $p$ generated by $\chi$. In the next two subsections, we extend the generation of paths in $p$ from paths in $q$, first to paths without slipknots, then to arbitrary paths.

4.2. Bridges and straight paths. Let $c=\left\langle t_{0} \mid s_{0}\right\rangle \in \mathbf{C}(p)$ with $t_{0}=\otimes(\vec{t})$ and $s_{0}=\not \mathcal{P}(\vec{s})$. We say an edge $e$ is bound to $c$ if either $e=c$, or $e=\left(t_{0}, t\right)$ with $t \in \vec{t}$, or $e=s_{0}$. And we say $\chi \in \mathbf{P}(p)$ is bound to $c$ if all the edges crossed by $\chi$ are bound to $c$. Observe that $e \in \mathbf{P}(p)$ is a preserved edge iff it is not bound to an eliminated cut.

Lemma 4.4. Let $c=\left\langle t_{0} \mid s_{0}\right\rangle$ :

- if $\chi \in \mathbf{P}(p)$ is bound to $c=\left\langle t_{0} \mid s_{0}\right\rangle$ and $s_{0}=\not(\vec{s})$ then there exists $s \in \vec{s}$ such that $\chi \in \mathbf{P}(p, I)$ whenever $I\left(s_{0}\right)=s$;

- if $\chi \in \mathbf{P}(p, I)$ does not cross any edge bound to $c$ then $\chi \in \mathbf{P}\left(p, I^{\prime}\right)$ whenever $I$ and $I^{\prime}$ differ only on $s_{0}$.

Proof. It is sufficient to observe that the edge $s_{0}$ is bound to $c$; and the only edge $e \in \mathbf{T}_{\not>}(p)$ that may be visited by a path bound to $c$ is $s_{0}$.

A $c$-bridge is a path $\chi$ that is bound to $c$ and that crosses $c$. Observe that $\chi$ is a $c$-bridge iff either $\chi$ or the reverse path $\chi^{\dagger}$ is a subpath of some $t \sim_{\left(t_{0}, t\right)} t_{0} \sim_{c} s_{0} \sim_{s_{0}} s$ with $t \in \vec{t}$ and $s \in \vec{s}$. Moreover, given $t \in\left\{t_{0}\right\} \cup \vec{t}$ and $s \in\left\{s_{0}\right\} \cup \vec{s}$ there is a unique $c$-bridge $t \rightsquigarrow s$. 
Lemma 4.5. Assume $\chi_{1} \xi \chi_{2} \in \mathbf{P}(q)$ and $\xi=t \sim_{\langle t \mid s\rangle} s$, where $\chi_{1}$ and $\chi_{2}$ are preserved paths, $t \in \vec{t}$ and $s \in \vec{s}$. Then there exists a c-bridge $\xi^{\simeq}$ such that $\chi_{1}^{-} \xi^{\simeq} \chi_{2}^{-} \in \mathbf{P}(p)$.

Proof. Write $\chi_{1}: v_{1} \rightsquigarrow q, J t$ and $\chi_{2}: s \rightsquigarrow q, J \quad v_{2}$ : by Lemma 4.3, we obtain $\chi_{1}^{-}: u_{1} \rightsquigarrow p, I t^{\prime}$ and $\chi_{2}^{-}: s^{\prime} \rightsquigarrow p, I u_{2}$ where $u_{1}, t^{\prime}, s^{\prime}$ and $u_{2}$ are anchors of $v_{1}, t, s$ and $v_{2}$ respectively, for any extension $I$ of $J$. In particular, $t^{\prime} \in\left\{t, t_{0}\right\}$ and $s^{\prime} \in\left\{s, s_{0}\right\}$ and we can fix $\xi^{\simeq}: t^{\prime} \rightsquigarrow s^{\prime}$ to be the only $c$-bridge with those endpoints. Observe indeed that $\xi^{\simeq} \in \mathbf{P}(p, I)$ as soon as $I\left(s_{0}\right)=s$. Then by Lemmas 4.3 and 4.4 , we can concatenate $\chi_{1}^{-} \xi^{\simeq} \chi_{2}^{-}: u_{1} \rightsquigarrow_{p, I} u_{2}$.

Despite the notation, the definition of $\xi^{\simeq}$ does depend on $\chi_{1}$ and $\chi_{2}$ : whenever we use Lemma 4.5, however, the values of $\chi_{1}$ and $\chi_{2}$ should be clear from the context.

We say a path $\chi \in \mathbf{P}(q)$ is a straight path if it has no slipknot. Such a path is essentially a path of $p$, up to replacing residuals with bridges:

Lemma 4.6. If $\chi$ is a straight path, there exists a unique sequence of pairwise distinct eliminated cuts $c_{1}, \ldots, c_{n} \in \mathbf{C}(p) \backslash \mathbf{C}(q)$, such that we can write $\chi=\chi_{1}\left(d_{1}\right) \cdots \chi_{n}\left(d_{n}\right) \chi_{n+1}$ where each $d_{i}$ is a residual of $c_{i}$ and $\chi$ crosses no other residual. Moreover we can form $\chi^{-}=\chi_{1}^{-}\left(d_{1}\right) \simeq \cdots \chi_{n}^{-}\left(d_{n}\right) \simeq \chi_{n+1}^{-} \in \mathbf{P}(p)$.

Proof. The first part is straightforward reformulation of the absence of slipknots. The second part follows by applying Lemma 4.5 to each $\chi_{i}\left(d_{i}\right) \chi_{i+1}$ (or the reverse path): the concatenation of preserved paths and bridges in the definition of $\chi^{-}$is allowed by Lemmas 4.3 and 4.4 .

We say two paths $\chi_{1}, \chi_{2} \in \mathbf{P}(q)$ are independent if they are disjoint and there is no eliminated cut $c$ such that both $\chi_{1}$ and $\chi_{2}$ cross a residual of $c$.

Lemma 4.7. Assume $\chi_{1}, \ldots, \chi_{n} \in \mathbf{P}(q, J)$ are pairwise independent straight paths and $c_{1}, \ldots, c_{k} \in \mathbf{C}(p) \backslash \mathbf{C}(q)$ are such that no $\chi_{i}$ crosses a residual of $c_{j}$, for $1 \leq i \leq n$ and $1 \leq j \leq k$. Then for any $\xi_{1}, \ldots, \xi_{k} \in \mathbf{P}(p)$ such that $\xi_{i}$ is bound to $c$ for $1 \leq i \leq k$, there exists an extension $I$ of $J$ such that $\chi_{i}^{-} \in \mathbf{P}(p, I)$ for $1 \leq i \leq n$ and $\xi_{j} \in \mathbf{P}(p, I)$ for $1 \leq j \leq k$.

Proof. It is sufficient to observe that if $\chi^{-}$crosses an edge bound to an eliminated cut $c$ then $\chi$ crosses a residual of $c$. Then the result is a direct consequence of the definition of $\chi_{i}^{-}$, together with Lemmas 4.3 and 4.4 .

The generation of a path is thus compatible with the concatenation of independent straight paths:

Lemma 4.8. If $\chi_{1}: v \rightsquigarrow_{q} u$ and $\chi_{2}: u \rightsquigarrow_{q} t$ are independent straight paths then $\chi_{1} \chi_{2}$ is a straight path and $\left(\chi_{1} \chi_{2}\right)^{-}=\chi_{1}^{-} \chi_{2}^{-}$.

Proof. That $\chi_{1} \chi_{2}$ is a straight path follows directly from the hypotheses. Write $\chi_{i}=$ $\chi_{1}^{i}\left(d_{1}^{i}\right) \cdots \chi_{n_{i}}^{i}\left(d_{n_{i}}^{i}\right) \chi_{n_{i}+1}^{i}$ : it is then sufficient to apply the definition of $\chi_{i}^{-}$and observe that $\left(\chi_{n_{1}+1}^{1} \chi_{1}^{2}\right)^{-}=\left(\chi_{n_{1}+1}^{1}\right)^{-}\left(\chi_{1}^{2}\right)^{-}$by Lemma 4.3 . 

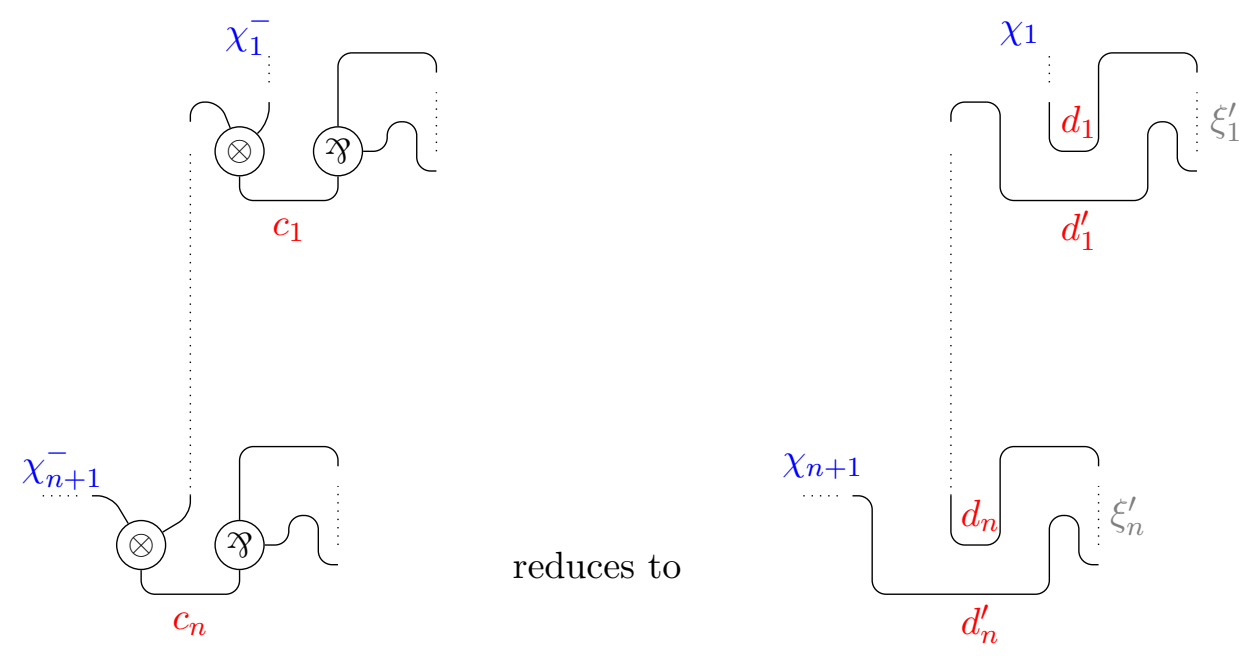

Figure 13: Schematic shape of slipknots on a path (axiom and cut nodes ommited)

4.3. Bounces and slipknots. Let $c=\left\langle t_{0} \mid s_{0}\right\rangle \in \mathbf{C}(p)$ with $t_{0}=\otimes(\vec{t})$. A $c$-bounce is a path $\chi$ that is bound to $c$, that does not $\operatorname{cross} c$ and that visits $t_{0}: \chi$ is either the empty path $\epsilon_{t_{0}}$, or $t_{0} \sim_{t_{0}, t} t$ or $t \sim_{t_{0}, t} t_{0}$ with $t \in \vec{t}$, or $t \sim_{\left(t_{0}, t\right)} t_{0} \sim_{\left(t_{0}, t^{\prime}\right)} t^{\prime}$ with $t \neq t^{\prime} \in \vec{t}$. Given $t, t^{\prime} \in\left\{t_{0}\right\} \cup \vec{t}$, such that either $t=t_{0}$ or $t^{\prime}=t_{0}$ or $t \neq t^{\prime}$, there is a unique $c$-bounce $t \rightsquigarrow t^{\prime}$.

Lemma 4.9. Assume $\chi_{1} \xi \chi_{2} \in \mathbf{P}(q)$ and $\xi=\left(t_{1} \sim_{\left\langle t_{1} \mid s_{1}\right\rangle} s_{1}\right) \xi^{\prime}\left(s_{2} \sim_{\left\langle t_{2} \mid s_{2}\right\rangle} t_{2}\right)$, where $\chi_{1}$ and $\chi_{2}$ are preserved paths, $t_{1}, t_{2} \in \vec{t}$ and $s_{1}, s_{2} \in \vec{s}$. Then there exists a c-bounce $\xi^{\vee}$ such that $\chi_{1}^{-} \xi^{\vee} \chi_{2}^{-} \in \mathbf{P}(p)$.

Proof. Necessarily, $\left\langle t_{1} \mid s_{1}\right\rangle \neq\left\langle t_{2} \mid s_{2}\right\rangle$, hence $t_{1} \neq t_{2}$. Write $\chi_{1}: v_{1} \rightsquigarrow_{q} t_{1}$ and $\chi_{2}: t_{2} \rightsquigarrow_{q} v_{2}$ : we obtain $\chi_{1}^{-}: u_{1} \rightsquigarrow p t_{1}^{\prime}$ and $\chi_{2}^{-}: t_{2}^{\prime} \rightsquigarrow p u_{2}$ where $u_{1}, t_{1}^{\prime}, t_{2}^{\prime}$ and $u_{2}$ are anchors of $v_{1}, t_{1}, t_{2}$ and $v_{2}$ respectively, for any extension $I$ of $J$. In particular, $t_{1}^{\prime} \in\left\{t_{1}, t_{0}\right\}$ and $t_{2}^{\prime} \in\left\{t_{2}, t_{0}\right\}$ with $t_{1} \neq t_{2}$, and we can fix $\xi^{\vee}: t_{1}^{\prime} \rightsquigarrow t_{2}^{\prime}$ to be the only the only $c$-bounce with those endpoints. Then we can concatenate $\chi_{1}^{-} \xi^{\vee} \chi_{2}^{-}: u_{1} \rightsquigarrow p u_{2}$ by Lemmas 4.3 and 4.4.

Again, the definition of $\xi^{\vee}$ does depend on $\chi_{1}$ and $\chi_{2}$ but these should be clear from the context when we use Lemma 4.9.

We are now ready to prove that paths in $q$ are alternations of straight paths and slipknots, and generate paths in $p$ by replacing slipknots with bounces:

Theorem 4.10. For each path $\chi \in \mathbf{P}(q)$, there exists a unique sequence of pairwise distinct eliminated cuts $c_{1}, \ldots, c_{n} \in \mathbf{C}(p) \backslash \mathbf{C}(q)$, such than we can write $\chi=\chi_{1} \xi_{1} \cdots \chi_{n} \xi_{n} \chi_{n+1}$ where:

- each $\chi_{i}$ is a straight path that crosses no residual of $c_{j}$ for $1 \leq j \leq n$;

- $\chi_{i}$ and $\chi_{j}$ are independent when $i \neq j$;

- each $\xi_{i}$ is a slipknot $\left(d_{i}\right) \xi_{i}^{\prime}\left(d_{i}^{\prime}\right): t_{i} \rightsquigarrow t_{i}^{\prime}$ where $d_{i}$ and $d_{i}^{\prime}$ are residuals of $c_{i}$, and $t_{i}$ and $t_{i}^{\prime}$ are distinct premises of the $\otimes$-tree of $c_{i}$.

Moreover $\chi^{-}=\chi_{1}^{-} \xi_{1}^{\vee} \cdots \chi_{n}^{-} \xi_{n}^{\vee} \chi_{n+1}^{-} \in \mathbf{P}(p)$. 
Figure 13 illustrates the relationship between $\chi^{-}$and $\chi$, in the simple case where each cut is between binary connectives, and no redirected jump is involved: each $\chi_{i}^{-}$bounces on the $\otimes$ side of $c_{i}$ and joins $\chi_{i+1}^{-}$directly instead of crossing the cut.

The proof of Theorem 4.10 is by induction on the length of $\chi$. We break it down into a series of intermediate results. Formally, given $\chi \in \mathbf{P}(q)$ :

- we first establish Lemma 4.11 for all paths such that Theorem 4.10 holds;

- we deduce Lemma 4.12 for $\chi$ from Theorem 4.10 and Lemma 4.11 applied to $\chi$;

- Lemma 4.13 for $\chi$ is a direct consequence of Lemma 4.12 applied to strict subpaths of $\chi$;

- we prove Lemma 4.14 for $\chi$ by applying Theorem 4.10, Lemma 4.11 and Lemma 4.13 to strict subpaths of $\chi$;

- then we prove Theorem 4.10 for $\chi$ by applying Lemmas 4.13 and 4.14 to $\chi$, and Lemma 4.11 to strict subpaths of $\chi$.

Lemma 4.11. Assume $\chi_{1}, \ldots, \chi_{n} \in \mathbf{P}(q, J)$ are pairwise disjoint paths, and $c_{1}, \ldots, c_{k} \in$ $\mathbf{C}(p) \backslash \mathbf{C}(q)$, are such that no $\chi_{i}$ crosses a residual of $c_{j}$, for $1 \leq i \leq n$ and $1 \leq j \leq k$. Then for any $\xi_{1}, \ldots, \xi_{k} \in \mathbf{P}(p)$ such that $\xi_{i}$ is bound to $c$ for $1 \leq i \leq k$, there exists an extension $I$ of $J$ such that $\chi_{i}^{-} \in \mathbf{P}(p, I)$ for $1 \leq i \leq n$ and $\xi_{j} \in \mathbf{P}(p, I)$ for $1 \leq j \leq k$.

Proof. This is a direct consequence of the definition of $\chi_{i}^{-}$in Theorem 4.10, together with Lemma 4.7.

Lemma 4.12. Assume $d=\langle t \mid s\rangle$ and $d^{\prime}=\left\langle t^{\prime} \mid s^{\prime}\right\rangle$ are distinct residuals of the same cut $c=\left\langle t_{0} \mid s_{0}\right\rangle$ with $t_{0}=\otimes(\vec{t}), s_{0}=\not \gamma(\vec{s}), t, t^{\prime} \in \vec{t}$ and $s, s^{\prime} \in \vec{s}$. If $u \in d, u^{\prime} \in d^{\prime}$ and $\chi: u \rightsquigarrow q u^{\prime}$ crosses no residual of $c$ then $u=s$ and $u^{\prime}=s^{\prime}$.

Proof. Write $\chi=\chi_{1} \xi_{1} \cdots \chi_{n} \xi_{n} \chi_{n+1}$ as in Theorem 4.10, and $\chi^{-}: v \rightsquigarrow p, I v^{\prime}$ where $v$ (resp. $\left.v^{\prime}\right)$ is an anchor of $u$ (resp. $u^{\prime}$ ). Observe that $\chi$ is non empty because $u \neq u^{\prime}$. Moreover, the first edge of $\chi$ is a preserved edge: if $\chi=\left(u \sim_{e} v\right) \chi^{\prime}$ then $e$ cannot be a cut, as otherwise we would have $e=d$ and $\chi$ would cross a residual of $c$. It follows that $\chi_{1}^{-}$is non empty, hence $\chi^{-}$is non empty. Since $\chi^{-}$is non empty, there is no path $\zeta: v \rightsquigarrow v^{\prime}$ bound to $c$ : otherwise, by Lemma 4.11, we could form a non empty cycle $\zeta^{\dagger} \chi^{-}: v^{\prime} \rightsquigarrow p v^{\prime}$.

If $u=t$ and $u^{\prime}=t^{\prime}$, we have $v \in\left\{t, t_{0}\right\}$ and $v^{\prime} \in\left\{t^{\prime}, t_{0}\right\}$ with $t \neq t^{\prime}$, and we obtain a $c$-bounce $v \rightsquigarrow v^{\prime}$, hence a contradiction. If $u=t$ and $u^{\prime}=s^{\prime}$, we have $v \in\left\{t, t_{0}\right\}$ and $v^{\prime} \in\left\{s^{\prime}, s_{0}\right\}$, and we obtain a $c$-bridge $v \rightsquigarrow v^{\prime}$, hence a contradiction. We rule out the case $u=s$ and $u^{\prime}=t^{\prime}$ symmetrically.

Lemma 4.13. If $\chi \in \mathbf{P}(q)$ and $c=\langle\otimes(\vec{t}) \mid \ngtr(\vec{s})\rangle \in \mathbf{C}(p) \backslash \mathbf{C}(q)$, then $\chi$ crosses at most two residuals of $c$, and in this case we can write $\chi=\chi_{1}\left(t \sim_{\langle t \mid s\rangle} s\right) \chi_{2}\left(s^{\prime} \sim_{\left\langle t^{\prime} \mid s^{\prime}\right\rangle} t^{\prime}\right) \chi_{3}$ with $t, t^{\prime} \in \vec{t}$ and $s, s^{\prime} \in \vec{s}$.

Proof. If $\chi=\chi_{1}(d) \chi_{2}\left(d^{\prime}\right) \chi_{3}$, where $d=\langle t \mid s\rangle$ and $d^{\prime}=\left\langle t^{\prime} \mid s^{\prime}\right\rangle$ are residuals of $c$ with $t, t^{\prime} \in \vec{t}$ and $s, s^{\prime} \in \vec{s}$, and if $\chi_{2}$ crosses no residual of $c$, then by Lemma 4.12 applied to $\chi_{2}$, we obtain $\chi=\chi_{1}\left(t \sim_{\langle t \mid s\rangle} s\right) \chi_{2}\left(s^{\prime} \sim_{\left\langle t^{\prime} \mid s^{\prime}\right\rangle} t^{\prime}\right) \chi_{3}$. If moreover $\chi_{1}$ (resp. $\left.\chi_{3}\right)$ crossed another residual of $c$, we would obtain a contradiction by applying Lemma 4.12 to a strict subpath of $\chi_{1}$ (resp. $\chi_{3}$ ) hence of $\chi$.

Lemma 4.14. Slipknots are well-bracketed in the following sense: there is no path $\chi=$ $\left(d_{1}\right) \chi_{1}\left(d_{2}\right) \chi_{2}\left(d_{1}^{\prime}\right) \chi_{3}\left(d_{2}^{\prime}\right) \in \mathbf{P}(q)$ such that, for $1 \leq i \leq 2, d_{i}$ and $d_{i}^{\prime}$ are residuals of the same cut. 
Proof. Assume $\chi=\left(d_{1}\right) \chi_{1}\left(d_{2}\right) \chi_{2}\left(d_{1}^{\prime}\right) \chi_{3}\left(d_{2}^{\prime}\right) \in \mathbf{P}(q)$ such that, for $1 \leq i \leq 2, d_{i}$ and $d_{i}^{\prime}$ are residuals of the same cut. We can assume w.l.o.g. that $\chi_{1}$ and $\chi_{3}$ are independent: otherwise there is a prefix of $\chi$ with this additional property.

For $1 \leq i \leq 2$, let $c_{i}=\left\langle t_{0}^{i} \mid s_{0}^{i}\right\rangle$, with $t_{0}^{i}=\otimes\left(\vec{t}_{i}\right)$ and $s_{0}^{i}=\varnothing\left(\vec{s}_{i}\right)$, and assume $d_{i}=\left\{t_{i}, s_{i}\right\}$ and $d_{i}^{\prime}=\left\{t_{i}^{\prime}, s_{i}^{\prime}\right\}$, with $t_{i}, t_{i}^{\prime} \in \vec{t}_{i}$ and $s_{i}, s_{i}^{\prime} \in \vec{s}_{i}$. By Lemma 4.13, we must have $\chi=\left(t_{1} \sim_{d_{1}} s_{1}\right) \chi_{1}\left(t_{2} \sim_{d_{2}} s_{2}\right) \chi_{2}\left(s_{1}^{\prime} \sim_{d_{1}^{\prime}} t_{1}^{\prime}\right) \chi_{3}\left(s_{2}^{\prime} \sim_{d_{2}^{\prime}} t_{2}^{\prime}\right)$.

By Theorem 4.10, we obtain $\chi_{1}^{-}: u_{1} \rightsquigarrow p v_{2}$ and $\chi_{3}^{-}: v_{1} \rightsquigarrow p u_{2}$, where $u_{1} \in\left\{s_{1}, s_{0}^{1}\right\}$, $v_{2} \in\left\{t_{2}, t_{0}^{2}\right\}, v_{1} \in\left\{t_{1}^{\prime}, t_{0}^{1}\right\}$ and $u_{2} \in\left\{s_{2}^{\prime}, s_{0}^{2}\right\}$. Write $\zeta_{1}: v_{1} \rightsquigarrow_{p} u_{1}$ (resp. $\zeta_{2}: v_{2} \rightsquigarrow_{p} u_{2}$ ) for the only $c_{1}$-bridge (resp. $c_{2}$-bridge) with those endpoints. Then we obtain a non empty cycle $\chi_{1}^{-} \zeta_{2}\left(\chi_{3}^{\dagger}\right)^{-} \zeta_{1}: u_{1} \rightsquigarrow p u_{1}$ : the concatenation is allowed by Lemma 4.11 applied to $\chi_{1}$ and $\chi_{3}^{\dagger}$.

Proof of Theorem 4.10. By Lemma 4.14, we can write $\chi=\chi_{1} \xi_{1} \cdots \chi_{n} \xi_{n} \chi_{n+1}$ where $\xi_{1}, \ldots, \xi_{n}$ are the slipknots of $\chi$ that are maximal (i.e. not strict subpaths of other slipknots) and this writing is unique. Let $c_{1}, \ldots, c_{n}$ be the associated eliminated cuts. By Lemma 4.13 , the $c_{j}$ 's are pairwise distinct, and $\xi_{j}: t_{j} \rightsquigarrow p t_{j}^{\prime}$ where $t_{j}$ and $t_{j}^{\prime}$ are distinct premises of the $\otimes$-tree of $c_{j}$. Moreover, since each slipknot of $\chi$ is a subpath of some $\xi_{j}$ :

- each $\chi_{i}$ is a straight path and it crosses no residual of any $c_{j}$;

- the $\chi_{i}$ 's are pairwise independent.

If $n=0$, we can set $\chi^{-}=\chi_{1}^{-}$. Otherwise, we apply Lemma 4.11 to the $\chi_{i}{ }^{\prime}$ 's, which are strict subpaths of $\chi$, which allows to concatenate $\chi^{-}=\chi_{1}^{-} \xi_{1}^{\vee} \cdots \chi_{n}^{-} \xi_{n}^{\vee} \chi_{n+1}^{-} \in \mathbf{P}(p)$.

Again, the construction of $\chi^{-}$is compatible with the concatenation of independent paths:

Lemma 4.15. If $\chi_{1} \chi_{2} \in \mathbf{P}(q)$ and $\chi_{1}$ and $\chi_{2}$ are independent, then $\left(\chi_{1} \chi_{2}\right)^{-}=\chi_{1}^{-} \chi_{2}^{-}$.

Proof. As for Lemma 4.8, this is a direct consequence of the definition of $\left(\chi_{1} \chi_{2}\right)^{-}, \chi_{1}^{-}$and $\chi_{2}^{-}$, this time using Lemma 4.8 to concatenate a straight suffix of $\chi_{1}$ and a straight prefix of $\chi_{2}$.

4.4. Bounding the growth of $\ln$. Now we show that we can bound $\ln (q)$ depending only on $\ln (p)$. We first need some basic properties relating the length of $\chi^{-}$with that of $\chi$.

Lemma 4.16. Let $\chi, \xi \in \mathbf{P}(q)$ and $\zeta \in \mathbf{P}(p)$ :

(1) if $\chi$ is preserved then $\ln \left(\chi^{-}\right)=\ln (\chi)$;

(2) if $\zeta$ is a bridge then $1 \leq \ln (\zeta) \leq 3$;

(3) if $\zeta$ is a bounce then $\ln (\zeta) \leq 2$;

(4) if $\chi$ is straight, then $\ln (\chi) \leq \ln \left(\chi^{-}\right) \leq 3 \ln (\chi)$;

(5) if $\xi$ is a prefix of $\chi$ and $\chi$ is straight then $\xi^{-}$is a prefix of $\chi^{-}$;

(6) if $\xi$ is a slipknot then $\ln (\xi) \geq 3$ and $\ln \left(\xi^{\vee}\right)<\ln (\xi)$;

(7) in general $\ln \left(\chi^{-}\right) \leq 3 \ln (\chi)$.

Proof. The first three properties are direct consequences of the definitions. Item (4) follows from (1) and (2). Item (5) follows from Lemma 4.8. Item (6) follows from Lemma 4.2 and (3). And item (7) follows from (4) and (6). 
Observe that in general, we do not have $\ln \left(\zeta^{-}\right) \leq \ln \left(\chi^{-}\right)$when $\zeta$ is a prefix of $\chi$ : $\zeta$ may enter an arbitrarily long slipknot of $\chi$ that is replaced by a single bounce in $\chi^{-}$. For this reason, we introduce the following notion: if $\chi \in \mathbf{P}(q)$, we define the width of $\chi$ (relative to the reduction $p \rightrightarrows_{m} q$ we consider) by $\operatorname{width}(\chi)=\max \left\{\ln \left(\zeta^{-}\right) \mid \zeta\right.$ prefix of $\left.\chi\right\}$.

Lemma 4.17. For any path $\chi \in \mathbf{P}(q), \ln \left(\chi^{-}\right) \leq \mathbf{w i d t h}(\chi) \leq \ln (p)$ and $\mathbf{w i d t h}(\chi) \leq 3 \ln (\chi)$. Moreover, if $\zeta$ is a prefix of $\chi$, we have $\boldsymbol{w i d t h}(\zeta) \leq \operatorname{width}(\chi)$. If moreover $\chi$ is straight, $\operatorname{width}(\chi)=\ln \left(\chi^{-}\right) \geq \ln (\chi)$.

Proof. We obtain $\ln \left(\chi^{-}\right) \leq \operatorname{width}(\chi) \leq \ln (p)$ and $\boldsymbol{w i d t h}(\zeta) \leq \operatorname{width}(\chi)$ directly from the definition of width. Item (7) of Lemma 4.16 gives $\operatorname{width}(\chi) \leq 3 \ln (\chi)$. If $\chi$ is straight $\operatorname{width}(\chi)=\ln \left(\chi^{-}\right) \geq \ln (\chi)$ follows from items (4) and (5) of Lemma 4.16.

Define $\varphi: \mathbf{N} \rightarrow \mathbf{N}$ inductively by $\varphi(0)=0$ and $\varphi(n)=n+(n+1)(\varphi(n-1)+2)$ if $n>0$. Observe that $n \leq \varphi(n) \leq \varphi(n+1)$.

Lemma 4.18. If $\chi \in \mathbf{P}(q)$ then $\ln (\chi) \leq \varphi($ width $(\chi))$.

Proof. The proof is by induction on $\operatorname{width}(\chi)$. If $\chi$ is straight then, by Lemma 4.17, $\ln (\chi) \leq \operatorname{width}(\chi) \leq \varphi(\operatorname{width}(\chi))$.

Write $\chi=\chi_{1} \xi_{1} \cdots \chi_{n} \xi_{n} \chi_{n+1}$ as in Theorem 4.10: we have $\ln (\chi)=\sum_{i=1}^{n+1} \ln \left(\chi_{i}\right)+$ $\sum_{j=1}^{n} \ln \left(\xi_{j}\right)$. Since $\chi^{-}=\chi_{1}^{-} \xi_{1}^{\vee} \cdots \chi_{n}^{-} \xi_{n}^{\vee} \chi_{n+1}^{-}$, we have $\sum_{i=1}^{n+1} \ln \left(\chi_{i}^{-}\right) \leq \ln \left(\chi^{-}\right)$. Since each $\chi_{i}$ is straight, we obtain $\ln (\chi) \leq \ln \left(\chi^{-}\right)+\sum_{j=1}^{n} \ln \left(\xi_{j}\right)$ from the previous inequality, by applying item (4) of Lemma 4.16.

Moreover observe that, by Lemma 4.2, $\chi_{i}$ is non empty for $1<i<n+1$. Hence $\ln \left(\chi^{-}\right) \geq n-1$, and we obtain $n \leq \operatorname{width}(\chi)+1$.

It remains to bound $\ln \left(\xi_{j}\right)$ for $1 \leq j \leq n$. We can write $\xi_{j}=\left(d_{j}\right) \chi_{j}^{\prime}\left(d_{j}^{\prime}\right)$ where $d_{j}$ and $d_{j}^{\prime}$ are the residuals of the cut $c_{j}$ associated with $\xi_{j}$. Let $\zeta_{j}^{\prime}$ be a prefix of $\chi_{j}^{\prime}$ and write $\zeta_{j}=\chi_{1} \xi_{1} \cdots \chi_{j}\left(d_{j}\right) \zeta_{j}^{\prime}$ which is a prefix of $\chi$. Observe that, by Theorem $4.10, c_{j}$ has no residual in $\zeta_{j}$ other than $d_{j}$, and $\chi_{1} \xi_{1} \cdots \chi_{j}\left(d_{j}\right)$ and $\zeta_{j}^{\prime}$ are independent. Hence $\chi_{j}\left(d_{j}\right)$ is straight and $\zeta_{j}^{-}=\chi_{1}^{-} \xi_{1}^{\vee} \cdots \chi_{j}^{-} d_{j}^{\simeq}\left(\zeta_{j}^{\prime}\right)^{-}$follows by Lemma 4.15. Since $\ln \left(d_{\tilde{j}}^{\widetilde{\sim}}\right) \geq 1$, we obtain $\ln \left(\left(\zeta_{j}^{\prime}\right)^{-}\right) \leq \ln \left(\zeta_{j}^{-}\right)-1 \leq \operatorname{width}(\chi)-1$.

Hence width $\left(\chi_{j}^{\prime}\right) \leq \operatorname{width}(\chi)-1$ : we apply the induction hypothesis and obtain $\ln \left(\chi_{j}^{\prime}\right) \leq \varphi\left(\operatorname{width}\left(\chi_{j}^{\prime}\right)\right) \leq \varphi(\operatorname{width}(\chi)-1)$ because $\varphi$ is monotonous. It follows that $\ln \left(\xi_{j}\right) \leq \varphi(\boldsymbol{w i d t h}(\chi)-1)+2$, and we conclude:

$$
\begin{aligned}
\ln (\chi) & \leq \ln \left(\chi^{-}\right)+\sum_{j=1}^{n} \ln \left(\xi_{j}\right) \\
& \leq \operatorname{width}(\chi)+(\operatorname{width}(\chi)+1)(\varphi(\operatorname{width}(\chi)-1)+2) \\
& \leq \varphi(\operatorname{width}(\chi))
\end{aligned}
$$

Using Lemma 4.17 again, we obtain:

Corollary 4.19. Let $p \rightrightarrows_{m} q$. Then, $\ln (q) \leq \varphi(\ln (p))$.

Notice that the previous result can be seen as a quantitative version of the preservation of acyclicity in proof nets under reduction. In the following example, we illustrate how acyclicity is mandatory for the existence of a function $\varphi$ as in Corollary 4.19. 

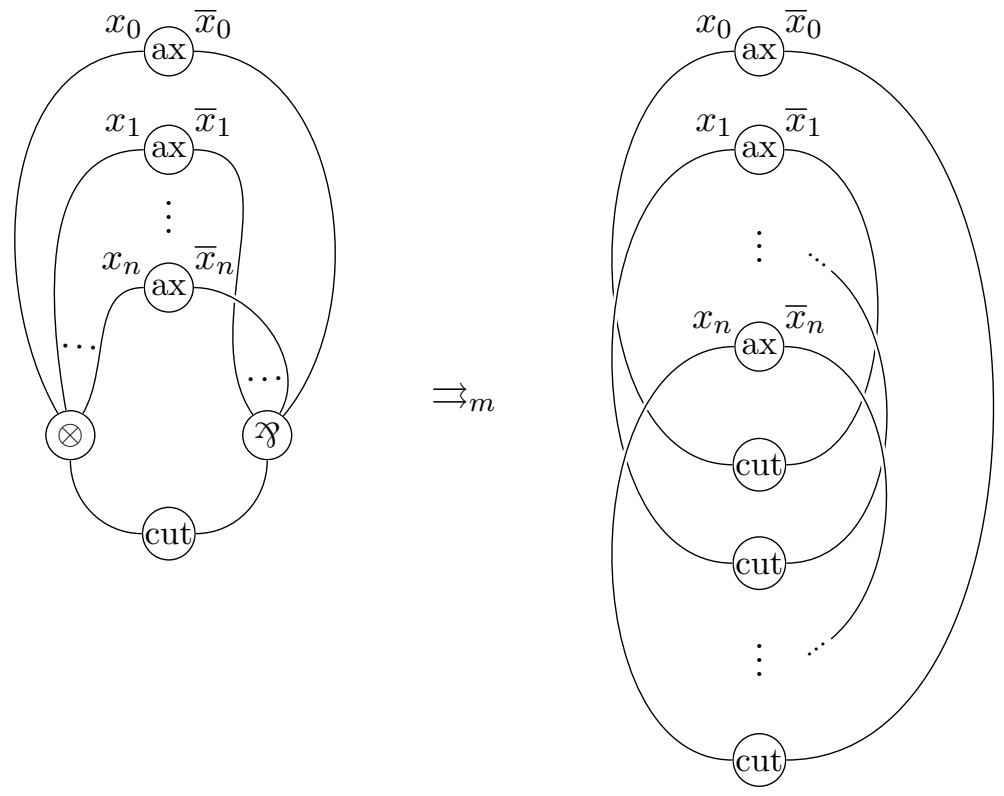

Figure 14: A cyclic counterexample to Corollary 4.19

Example 4.20. Let $p=(\langle t \mid s\rangle ;)$ with $t=\otimes\left(x_{0}, \ldots, x_{n}\right)$ and $s=\not \partial\left(\bar{x}_{1}, \ldots, \bar{x}_{n}, \bar{x}_{0}\right)$ : by setting $I(s)=\bar{x}_{i}$, we obtain a cycle $x_{i} \sim_{\left\{x_{i}, \bar{x}_{i}\right\}} \bar{x}_{i} \sim_{s} s \sim_{\langle t \mid s\rangle} t \sim_{t, x_{i}} x_{i}$. Since each path $\chi \in \mathbf{P}(p)$ can cross each of $s$ and $\langle t \mid s\rangle$ at most once, it is easy to check that $\ln (p)=6$.

But $p \rightrightarrows_{m} q=\left(\left\langle x_{0} \mid \bar{x}_{1}\right\rangle, \ldots,\left\langle x_{n-1} \mid \bar{x}_{n}\right\rangle,\left\langle x_{n} \mid \bar{x}_{0}\right\rangle ;\right)$ hence $\ln (q)=2(n+1)$. The situation is illustrated in Figure 14.

4.5. Erratum. In the extended abstract of the present paper, the analogue [CVA18, Subsection 3.2] of Subsection 4.4 claimed to establish similar results for another measure on paths: rather than the length $\ln (\xi)$ of a path $\xi$, we considered the number $\mathbf{c c}(\xi)$ of all the cuts $\langle t \mid s\rangle$ such that $\xi$ visits $t$ or $s$.

It is easy to check that Lemma 3.2 still holds if we replace the maximal length of a path with the maximum number of cuts in a chain of axiom cuts in the sense of Figure 2 . Given the situation depicted in Figure 13, however, it is evident that a bound on the number of cuts crossed by a path cannot be preserved: the path on the left hand side crosses no cut, while the path in the reduct crosses an arbitrary number of (possibly axiom) cuts. We introduced $\mathbf{c c}(\xi)$ in our previous attempt, precisely to capture this example: if $\xi$ is the path following the tensors of the left hand side, then $\mathbf{c c}(\xi) \geq n$. But this fix is actually not sufficient: if we replace each $c_{i}=\left\langle t_{i} \mid s_{i}\right\rangle$ in Figure 13, with $\left\langle\left.\otimes\left(t_{i}, s_{i}\right)\right|^{\gamma}\left(x_{i}, \overline{x_{i}}\right)\right\rangle$, a path in the obtained net can visit at most two of these new cuts, but it reduces to the left hand side, with $\xi$ such that $\mathbf{c c}(\xi) \geq n$.

It might be possible to adapt our method for dealing with a relaxed definition of visited cut: for instance, we might consider the number of cuts $c$ such that $\xi$ visits a tree $t \in \mathbf{T}(c)$ (instead of $t \in c$ ). But this notion is no longer local, and would introduce further technicalities: for that reason, we decided to focus on the length of paths instead, which is a more intuitive and standard notion, without any ad hoc reference to cuts. 


\section{VARIATIONS OF $\mathbf{j d}(p)$ UNDER REDUCTION}

For establishing that $\mathbf{j d}(q)$ is bounded as a function of $\mathbf{j} \mathbf{d}(p)$ and $\ln (p)$ we examine the reductions separately.

Lemma 5.1. Let $p, q$ two proof nets. If $p \rightrightarrows_{m} q$, then $\mathbf{j d}(q) \leq 2 \mathbf{j d}(p)$.

Proof. For all $t \in \mathbf{T}(q)$ the only case in which $\jmath_{q}^{-1}(t) \neq j_{p}^{-1}(t)$ is that of redirected jumps: there must be $\mu \in \mathbf{U}_{\perp}(q)$ such that $t$ is part of a residual $\langle t \mid s\rangle$ of an eliminated cut $\left\langle t_{0} \mid s_{0}\right\rangle \in \mathbf{C}(p)$, with $\jmath_{p}(\mu)=t_{0}$. In this case we have $\mathbf{j d}_{q}(t)=\mathbf{j} \mathbf{d}_{p}(t)+\mathbf{j} \mathbf{d}_{p}\left(t_{0}\right) \leq 2 \mathbf{j} \mathbf{d}(p)$.

Lemma 5.2. Let $p, q$ two proof nets. If $p \rightrightarrows_{e} q$, then $\mathbf{j d}(q) \leq(2 \mathbf{j} \mathbf{d}(p))^{\mathbf{l n}(p)+1}$.

Proof. Fix $t \in \mathbf{T}(q)$. For any $\mu \in \mathbf{U}_{\perp}(q)$, if $\jmath_{q}(\mu)=t$, then $\mu \in \mathbf{I}_{p}^{n}(t)$ for some $n \in \mathbf{N}$ : this is precisely the purpose of the definition of $\mathbf{I}_{p}^{n}$. We obtain $\mathbf{j d}_{q}(t) \leq \#\left(\bigcup_{n \in \mathbf{N}} \mathbf{I}_{p}^{n}(t)\right) \leq$ $(2 \mathbf{j d}(p))^{\ln (p)+1}$ by Lemma $3.4(1)$.

Lemma 5.3. Let $p, q$ two proof nets. If $p \rightrightarrows_{a} q$, then $\mathbf{j d}(q) \leq(\ln (p)+1) \mathbf{j d}(p)$.

Proof. As in the proof of Lemma 3.2, we can write $p=\left(\overrightarrow{c_{1}}, \ldots, \overrightarrow{c_{k}}, \vec{c} ; \vec{s}\right)$ and $q=$ $(\vec{c} ; \vec{s})\left[t^{1} / \bar{x}_{0}^{1}\right] \cdots\left[t^{k} / \bar{x}_{0}^{k}\right]$ where $\vec{c}_{i}=\left(\left\langle x_{0}^{i} \mid \bar{x}_{1}^{i}\right\rangle, \ldots,\left\langle x_{n_{i}-1}^{i} \mid \bar{x}_{n_{i}}^{i}\right\rangle,\left\langle x_{n_{i}}^{i} \mid t^{i}\right\rangle\right)$.

By the definition of cut elimination, for all $\mu \in \mathbf{U}_{\perp}(q)$ we have:

$$
\jmath_{q}(\mu)=\left\{\begin{array}{ll}
t^{i}\left[t^{i+1} / \bar{x}_{0}^{i+1}\right] \cdots\left[t^{k} / \bar{x}_{0}^{k}\right] & \text { if } \jmath_{p}(\mu) \in\left\{x_{0}^{i}, \bar{x}_{0}^{i}, x_{1}^{i}, \ldots, \bar{x}_{n_{i}}^{i}, t^{i}\right\} \\
\jmath_{q}(\mu)\left[t^{1} / \bar{x}_{0}^{1}\right] \cdots\left[t^{k} / \bar{x}_{0}^{k}\right] & \text { otherwise }
\end{array} .\right.
$$

It follows that:

- $\mathbf{j d}_{q}\left(t^{i}\left[t^{i+1} / \bar{x}_{0}^{i+1}\right] \cdots\left[t^{k} / \bar{x}_{0}^{k}\right]\right)=\mathbf{j d}_{p}\left(t^{i}\right)+\sum_{j=0}^{n_{i}}\left(\mathbf{j d}_{p}\left(x_{j}^{i}\right)+\mathbf{j d}_{p}\left(\bar{x}_{j}^{i}\right)\right) \leq\left(2 n_{i}+3\right) \mathbf{j d}(p)$;

- if $t \in \mathbf{T}(p) \backslash \bigcup_{i=1}^{k}\left\{x_{0}^{i}, \bar{x}_{0}^{i}, x_{1}^{i}, \ldots, \bar{x}_{n_{i}}^{i}, t^{i}\right\}$, then $\mathbf{j d}_{q}\left(t\left[t^{1} / \bar{x}_{0}^{1}\right] \cdots\left[t^{k} / \bar{x}_{0}^{k}\right]\right)=\mathbf{j d}_{p}(t)$.

To conclude, it is sufficient to observe that $2 n_{i}+2 \leq \ln (p)$ : indeed each $\vec{c}_{i}$ induces a path alternating between $n_{i}+1$ axioms an $n_{i}+1$ cuts.

\section{Bounding THE SIZE OF ANTIREDUCTS: GENERAL AND ITERATED CASE}

The previous results now allow us to treat the general case of a reduction $p \rightrightarrows q$.

Theorem 6.1. If $p \rightrightarrows q$ then $\operatorname{size}(p) \leq \psi(2(\ln (p)+1) \operatorname{size}(q), \ln (p), \mathbf{j d}(p))$.

Proof. Consider $q^{\prime}, q^{\prime \prime}$ such that $p \rightrightarrows_{e} q^{\prime} \rightrightarrows_{a} q^{\prime \prime} \rightrightarrows_{m} q$. We have:

$$
\begin{aligned}
\operatorname{size}(p) & \leq \psi\left(\operatorname{size}\left(q^{\prime}\right), \mathbf{j d}(p), \ln (p)\right) \\
& \leq \psi\left(\left(\ln \left(q^{\prime}\right)+1\right) \operatorname{size}\left(q^{\prime \prime}\right), \mathbf{j d}(p), \ln (p)\right) \\
& \leq \psi\left((\ln (p)+1) \operatorname{size}\left(q^{\prime \prime}\right), \mathbf{j d}(p), \ln (p)\right) \\
& \leq \psi(2(\ln (p)+1) \operatorname{size}(q), \mathbf{j d}(p), \ln (p))
\end{aligned}
$$

Corollary 6.2. If $q$ is an $M L L$ net and $n, m \in \mathbf{N}$, then

$$
\{p \mid p \rightrightarrows q, \mathbf{j d}(p) \leq m \text { and } \ln (p) \leq n\}
$$

is finite. 
Of course, that result holds only up to the $\alpha$-equivalence mentioned in Remark 2.1: then it is easy to check that the cardinality of $\{p \mid \operatorname{size}(p) \leq k\}$ is bounded by a function of $k \in \mathbf{N}$. Also recall from Remark 2.2 that we are actually interested in bare nets rather than nets with jumps, so Corollary 6.2 should be read as follows: given a bare net $q$ and $n, m \in \mathbf{N}$ there are finitely many bare nets $p$ such that $p \rightrightarrows q$ and that can be equipped with a jump function $\jmath_{p}$ satisfying $\mathbf{j d}(p) \leq m$ and $\ln (p) \leq n$. More precisely, Theorem 6.1 entails that the number of such bare nets $p$ can be bounded by a function of $m, n$ and $\operatorname{size}(q)$.

It follows that, given an infinite linear combination $\sum_{i \in I} a_{i} \cdot p_{i}$, assuming that we can equip each $p_{i}$ with a jump function $\jmath_{p_{i}}$ so that $\left\{\ln \left(p_{i}\right) \mid i \in I\right\} \cup\left\{\mathbf{j d}\left(p_{i}\right) \mid i \in I\right\}$ is finite, we can always consider an arbitrary family of reductions $p_{i} \rightrightarrows q_{i}$ for $i \in I$ and form the sum $\sum_{i \in I} a_{i} . q_{i}$ : this is always well defined. But if we want to iterate this process and perform a reduction from $\sum_{i \in I} a_{i} . q_{i}$ to $\sum_{i \in I} a_{i} . r_{i}$, when $q_{i} \rightrightarrows r_{i}$ for $i \in I$, we need to ensure that a similar side condition holds for the $q_{i}$ 's. Again, this is a consequence of our previous results, which we sum up in the following two theorems.

Theorem 6.3. Let $p \rightrightarrows q$. Then $\ln (q) \leq \varphi(\ln (p))$.

Proof. Consider $q^{\prime}, q^{\prime \prime}$ such that $p \rightrightarrows_{m} q^{\prime} \rightrightarrows_{e} q^{\prime \prime} \rightrightarrows_{a} q$. We have:

$$
\begin{aligned}
\ln (q) & \leq \ln \left(q^{\prime \prime}\right) & & (\text { by Lemma } 4.1) \\
& \leq \ln \left(q^{\prime}\right) & & (\text { by Lemma } 4.1) \\
& \leq \varphi(\ln (p)) & & (\text { by Corollary } 4.19)
\end{aligned}
$$

Theorem 6.4. There exists a function $\theta: \mathbf{N} \rightarrow \mathbf{N}$ such that $\mathbf{j d}(q) \leq \theta(\ln (p), \mathbf{j d}(p))$ whenever $p \rightrightarrows q$.

Proof. Consider $q^{\prime}, q^{\prime \prime}$ such that $p \rightrightarrows_{a} q^{\prime} \rightrightarrows_{e} q^{\prime \prime} \rightrightarrows_{m} q$. We have

$$
\begin{aligned}
\mathbf{j d}(q) & \leq 2 \mathbf{j d}\left(q^{\prime \prime}\right) & & (\text { by Lemma } 5.1) \\
& \leq 2\left(2 \mathbf{j d}\left(q^{\prime}\right)\right)^{\ln \left(q^{\prime}\right)+1} & & (\text { by Lemma } 5.2) \\
& \leq 2(2(\ln (p)+1) \mathbf{j d}(p))^{\ln \left(q^{\prime}\right)+1} & & (\text { by Lemma } 5.3) \\
& \leq 2(2(\ln (p)+1) \mathbf{j d}(p))^{\ln (p)+1} & & (\text { by Lemma } 4.1)
\end{aligned} .
$$

By the previous results, we can iterate Corollary 6.2 and obtain:

Corollary 6.5. If $q$ is an $M L L$ net and $k, n, m \in \mathbf{N}$, then

$$
\left\{p \mid p \rightrightarrows^{k} q, \mathbf{j d}(p) \leq m \text { and } \ln (p) \leq n\right\}
$$

is finite.

\section{TAYLOR EXPANSION}

We now show how the previous results apply to Taylor expansion. For that purpose, we must extend our syntax to MELL proof nets. Our presentation departs from Ehrhard's [Ehr16] in our treatment of promotion boxes: instead of introducing boxes as tree constructors labelled by nets, with auxiliary ports as inputs, we consider box ports as 0-ary trees, that are related with each other in a box context, associating each box with its contents. This is in accordance with the usual presentation of promotion as a black box, and has two motivations: 
- in Ehrhard's syntax, the promotion is not a net but an open tree, for which the trees associated with auxiliary ports must be mentioned explicitly: this would complicate the expression of Taylor expansion;

- since we consider a single class of ?-links instead of having a separate dereliction, we must impose constraints on auxiliary ports, that are easier to express when these ports are directly represented in the syntax.

Then we show that if $p$ is a resource net in the support of the Taylor expansion of an MELL proof net $P$, then $\ln (p)$ and $\mathbf{j d}(p)$ are bounded by functions of $P$.

Observe that we need only to consider the support of Taylor expansion, so we do not formalize the expansion of MELL nets into infinite linear combinations of resource nets: rather, we introduce $\mathcal{T}(P)$ as a set of approximants.

7.1. MELL nets. In addition to the set of variables, we fix a denumerable set $\mathbf{B}$ of box ports: we assume given an enumeration $\mathbf{B}=\left\{a_{i}^{b} \mid i, b \in \mathbf{N}\right\}$. We call principal ports the ports $a_{0}^{b}$ and auxiliary ports the other ports. Instead of separate contractions and derelictions, we consider a unified ?-link of arbitrary arity; auxiliary ports of boxes must be premises of such links (or of auxiliary ports of outer boxes, that must satisfy this constraint inductively).

The weakenings (and coweakenings, in the resource nets yet to be introduced) are not essentially different from the multiplicative units in our untyped nets. Indeed, we will see that the geometrical and combinatorial behaviour of the ?-link (resp. the !-link) is identical to that of the 8 (respectively, of the $\otimes$ ). This will be reflected in our use of labels: in addition to $\mathbf{U}_{\mathbf{1}}$ and $\mathbf{U}_{\perp}$, we will use labels from denumerable sets $\mathbf{U}_{\text {! }}$ and $\mathbf{U}_{\text {? }}$ (now assuming $\mathbf{V}, \mathbf{B}$, $\mathbf{U}_{\mathbf{1}}, \mathbf{U}_{\perp}, \mathbf{U}_{\text {! }}$ and $\mathbf{U}_{\text {? }}$ are pairwise disjoint), and write $\mathbf{U}_{+}=\mathbf{U}_{\mathbf{1}} \cup \mathbf{U}_{\text {! }}$ and $\mathbf{U}_{-}=\mathbf{U}_{\perp} \cup \mathbf{U}_{\text {? }}$.

We introduce the corresponding term syntax. Raw pre-trees $\left(S^{\circ}, T^{\circ}\right.$, etc.) and raw trees $(S, T$, etc.) are defined by mutual induction as follows:

$$
\begin{aligned}
& T:=x\left|\mathbf{1}_{\lambda}\right| \perp_{\mu}\left|\otimes\left(T_{1}, \ldots, T_{n}\right)\right| \mathcal{P}\left(T_{1}, \ldots, T_{n}\right)\left|a_{0}^{b}\right| ?_{\mu^{\prime}}() \mid ?\left(T_{1}^{\circ}, \ldots, T_{n}^{\circ}\right) \\
& T^{\circ}::=T \mid a_{i+1}^{b}
\end{aligned}
$$

where $x$ ranges over $\mathbf{V}, \lambda$ ranges over $\mathbf{U}_{1}, \mu$ ranges over $\mathbf{U}_{\perp}, \mu^{\prime}$ ranges over $\mathbf{U}_{?}, b$ and $i$ range over $\mathbf{N}$ and we require $n \neq 0$ in each of $\otimes\left(T_{1}, \ldots, T_{n}\right), \gamma\left(T_{1}, \ldots, T_{n}\right)$ and $?\left(T_{1}^{\circ}, \ldots, T_{n}^{\circ}\right)$. The set $\mathbf{T}^{\circ}(S)$ of the sub-pre-trees of $S$ is defined in the natural way, as well as the set $\mathbf{T}(S)$ of sub-trees of $S$, from which we derive the definitions of $\mathbf{V}(S), \mathbf{B}(S), \mathbf{U}_{\perp}(S)$, etc. The set $\mathbf{A}(S)$ of atoms of $S$ is then $\mathbf{V}(S) \cup \mathbf{B}(S) \cup \mathbf{U}_{\mathbf{1}}(S) \cup \mathbf{U}_{-}(S)$.

A tree (resp. a pre-tree) is a raw tree (resp. raw pre-tree) in which each atom occurs at most once. A cut is an unordered pair of trees $C=\langle T \mid S\rangle$ with disjoints sets of atoms. Pre-trees and cuts only describe the surface level of MELL nets: we also have to introduce promotion boxes.

We now define box contexts and pre-nets by mutual induction as follows. A box context $\Theta$ is the data of a finite set $B_{\Theta} \subset \mathbf{N}$, and of a pre-net of the form $\Theta(b)=\left(\Theta_{b} ; \vec{C}_{b} ; T_{b}, \vec{S}_{b}^{\circ} ; \jmath_{b}\right)$, for each $b \in B_{\Theta}$. We then write $\operatorname{ar}_{\Theta}(b)$, or simply $\operatorname{ar}(b)$ for the length of the family $\vec{S}_{b}^{\circ}$, which we call the arity of the box $b$. A pre-net is a tuple $P^{\circ}=\left(\Theta ; \vec{C} ; \vec{S}^{\circ} ; \jmath\right)$ where:

- $\Theta$ is a box context;

- the jump function $\mathrm{\jmath}$ is a function $\mathbf{U}_{-}\left(\vec{C}, \vec{S}^{\circ}\right) \rightarrow \mathbf{T}\left(\vec{C}, \vec{S}^{\circ}\right)$;

- each atom occurs at most once in $\vec{C}, \vec{S}^{\circ}$;

- $a_{i}^{b} \in \mathbf{B}\left(\vec{C} ; \vec{S}^{\circ}\right)$ iff $b \in B_{\Theta}$ and $0 \leq i \leq \operatorname{ar}(b)$; 
- $\mathbf{V}\left(\vec{C}, \vec{S}^{\circ}\right)$ is closed under the involution $x \mapsto \bar{x}$.

Then a net is a pre-net of the form $P=(\Theta ; \vec{C} ; \vec{S} ; \jmath)$, i.e. without auxiliary ports as conclusions. In the following, we may write, e.g., $\Theta_{P}$ for $\Theta$ in this case. An example is illustrated in Figure 15.

Remark 7.1. To be formal, in the definition of a box context $\Theta$, we should also fix an enumeration of the family $\vec{S}_{b}^{\circ}$ in $\Theta(b)$. Indeed, when we write $a_{0}^{b}, a_{1}^{b}, \ldots, a_{\operatorname{ar}(b)}^{b}$ for the ports of a box $b$, and $\Theta(b)=\left(\Theta_{b} ; \vec{C}_{b} ; T_{b}, S_{b, 1}^{\circ}, \ldots, S_{b, \operatorname{ar}(b)}^{\circ} ; \jmath_{b}\right)$ for the contents of the box, we implicitly assume a bijection which maps each pre-tree $S_{b, i}^{\circ}$ to the auxiliary port $a_{i}^{b}$ of which it is a premise (which leaves $T$ to be mapped to $a_{0}^{b}$ ). We prefer to keep this information implicit in the following, as the notations should allow to recover it, whenever necessary.

On the other hand, an analogue of Remark 2.1 applies in this new setting, as pre-nets and nets should be considered up to some notion of isomorphism preserving the interface, which amounts to:

- reindexing cuts, so that $\vec{C}$ is considered as a set;

- reordering premises of ?-links, which accounts for the associativity and commutativity of the underlying binary contraction;

- renaming atoms and boxes and, simultaneously, changing the enumeration of the family $\vec{S}_{b}^{\circ}$ in each box, all this preserving the duality involution on variables, the partition $\left\{a_{i}^{b} \mid i \in \mathbf{N}\right\}_{b \in \mathbf{N}}$ of $\mathbf{B}$, the jump functions, and the association of each $S_{b, i+1}^{\circ}$ to $a_{i+1}^{b}$.

We still consider this as a form of $\alpha$-equivalence as it only involves particular renamings of atoms or indices, preserving the rest of the structure. Again, we keep this quotient implicit whenever possible in the remaining.

Also, as already mentioned in Remark 2.2, we rely on jumps to control the combinatorics of the elimination of evanescent cuts: we need nets to be equipped with jumps only to ensure that the resource nets in the Taylor expansion can also be equipped with jumps, that moreover enjoy uniform bounds. More precisely, we will show that if an MELL net $P$ can be equipped with $\jmath_{P}$ that satisfies the acyclicity criterion, then each $p \in \mathcal{T}(P)$ can be equipped with $\jmath_{p}$, satisfying uniform bounds on $\ln (p)$ and $\mathbf{j d}(p)$.

The existence of such a jump function $\jmath_{P}$ should be understood as side condition only: we keep it in the definition of nets by default because we rely on it everywhere in the following, but in the end we are actually interested in the compatibility of Taylor expansion with cut elimination for nets without jumps. And the reader may check that, without jumps, our pre-nets (up to $\alpha$-equivalence) are essentially the same as, e.g., the in-PS's (up to the names of internal ports) defined by de Carvalho [dC16] for his proof of the injectivity of Taylor expansion.

Given a pre-net $P^{\circ}=\left(\Theta ; \vec{C} ; \vec{S}^{\circ} ; \jmath\right)$, we write $\mathbf{V}\left(P^{\circ}\right)=\mathbf{V}\left(\vec{C} ; \vec{S}^{\circ}\right), \mathbf{T}\left(P^{\circ}\right)=\mathbf{T}\left(\vec{C} ; \vec{S}^{\circ}\right)$, etc. We define the toplevel size of MELL pre-nets by $\operatorname{size}_{0}\left(P^{\circ}\right)=\# \mathbf{T}^{\circ}\left(P^{\circ}\right)$. We write $\operatorname{depth}\left(P^{\circ}\right)$ for the maximum level of nesting of boxes in $P^{\circ}$, i.e. the inductive depth in the above definition of pre-nets. The size of MELL pre-nets includes that of their boxes: we set $\operatorname{size}\left(P^{\circ}\right)=\operatorname{size}_{0}\left(P^{\circ}\right)+\sum_{b \in B_{\Theta}} \operatorname{size}(\Theta(b))$ - this definition is of course by induction on $\operatorname{depth}\left(P^{\circ}\right)$.

Notice that, by the above definition, for all $\mu \in \mathbf{U}_{-}\left(\vec{C}, \vec{S}^{\circ}\right), \jmath_{P^{\circ}}(\mu)$ must be at the same depth as $\mu$, and cannot be an auxiliary port. 


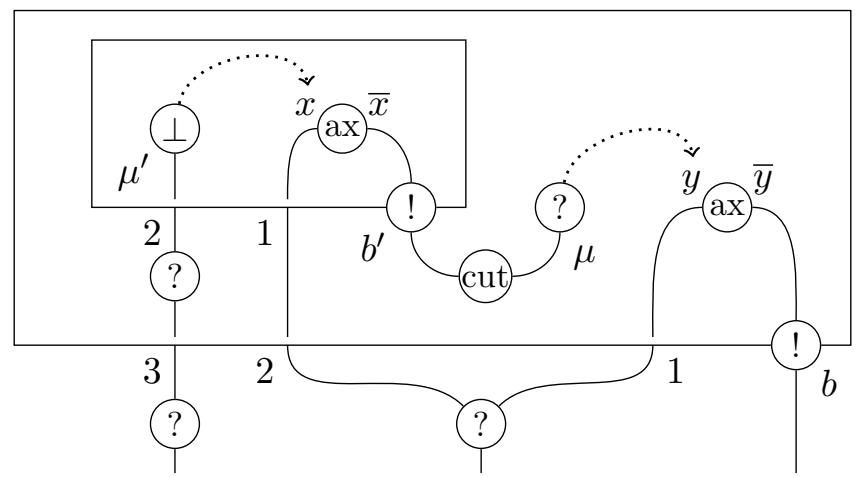

Figure 15: Representation of the net $\left(\Theta ; ; ?\left(a_{3}^{b}\right), ?\left(a_{2}^{b}, a_{1}^{b}\right), a_{0}^{b} ; \jmath\right)$ where $B_{\Theta}=\{b\}, \operatorname{ar}(b)=3$ and $\Theta(b)=\left(\Theta^{\prime} ;\left\langle a_{0}^{b^{\prime}} \mid \mu\right\rangle ; \bar{y}, y, a_{1}^{b^{\prime}}, ?\left(a_{2}^{b^{\prime}}\right) ; \jmath^{\prime}\right)$ where $B_{\Theta^{\prime}}=\left\{b^{\prime}\right\}, \operatorname{ar}\left(b^{\prime}\right)=2, \jmath^{\prime}(\mu)=y$ and $\Theta^{\prime}\left(b^{\prime}\right)=\left(\Theta^{\prime \prime} ; ; \bar{x}, x, \mu^{\prime} ; j^{\prime \prime}\right)$ where $B_{\Theta^{\prime \prime}}=\emptyset$ and $\jmath^{\prime \prime}\left(\mu^{\prime}\right)=x$.

We extend the switching functions of MLL to ?-links: for each $T=?\left(T_{1}^{\circ}, \ldots, T_{n}^{\circ}\right) \in$ $\mathbf{T}\left(P^{\circ}\right), I(T) \in\left\{T_{1}^{\circ}, \ldots, T_{n}^{\circ}\right\}$, which induces a ?-edge $T \sim_{T}^{P^{\circ}, I} I(T)$. We also consider box edges $a_{0}^{b} \sim_{b, i}^{P^{\circ}} a_{i}^{b}$ for $b \in B_{\Theta}$ and $1 \leq i \leq \operatorname{ar}(b)$ : w.r.t. paths, a box $b$ behaves like $\operatorname{ar}(b)$ axiom links having the principal port of the box as a common vertice, and the content is not considered. Finally, jump edges also include the case of weakenings: $\mu \sim_{\mu}^{P^{\circ}} \jmath(\mu)$ for $\mu \in \mathbf{U}_{-}\left(P^{\circ}\right)$.

We write $\mathbf{P}\left(P^{\circ}, I\right)$ (resp. $\left.\mathbf{P}\left(P^{\circ}\right)\right)$ for the set of $I$-paths (resp. paths) in $P^{\circ}$. We say a pre-net $P^{\circ}$ is acyclic if there is no cycle in $\mathbf{P}\left(P^{\circ}\right)$ and, inductively, each $\Theta(b)$ is acyclic. From now on, we consider acyclic pre-nets only.

7.2. Resource nets and Taylor expansion. The Taylor expansion of a net $P$ will be a set of resource nets: these are the same as the multiplicative nets introduced before, with the addition of term constructors for ! and ?. Raw trees are given as follows:

$$
t::=x\left|\mathbf{1}_{\lambda}\right| \perp_{\mu}\left|\otimes\left(t_{1}, \ldots, t_{n}\right)\right| \not \partial\left(t_{1}, \ldots, t_{n}\right)\left|!_{\lambda^{\prime}}()\right| ?_{\mu^{\prime}}()\left|!\left(t_{1}, \ldots, t_{n}\right)\right| ?\left(t_{1}, \ldots, t_{n}\right) .
$$

where $x$ ranges in $\mathbf{V}, \lambda$ ranges over $\mathbf{U}_{1}, \mu$ ranges over $\mathbf{U}_{\perp}, \lambda^{\prime}$ ranges over $\mathbf{U}_{!}, \mu^{\prime}$ ranges over $\mathbf{U}_{?}$, and we require $n \neq 0$ in each case. In resource nets, we extend switchings to ?-links and jumps from weakenings as in MELL nets, associated with ?-edges and jump edges. Moreover, for each $t=!\left(t_{1}, \ldots, t_{n}\right)$, we have !-edges $t \sim_{t, t_{i}} t_{i}$ for $1 \leq i \leq n$. Observe that, except for the notation of the root constructor, the trees $!_{\lambda}(), ?_{\mu}(), !\left(t_{1}, \ldots, t_{n}\right)$ and $?\left(t_{1}, \ldots, t_{n}\right)$, are exactly the same as $\mathbf{1}_{\lambda}, \perp_{\mu}, \otimes\left(t_{1}, \ldots, t_{n}\right)$ and $\mathcal{\gamma}\left(t_{1}, \ldots, t_{n}\right)$ respectively: in particular they induce the same geometry for paths.

During Taylor expansion, we need to replace a box in a pre-net with an arbitrary number of approximants of this box. Let us call box replacement of arity $n$ the data $r=\left(\vec{s}_{0}, \ldots, \vec{s}_{n}\right)$ of $n+1$ families of pairwise distinct resource trees $\vec{s}_{0}, \ldots, \vec{s}_{n}$, such that $\mathbf{A}(s) \cap \mathbf{A}\left(s^{\prime}\right)=\emptyset$ whenever $s \in \vec{s}_{i}$ and $s^{\prime} \in \vec{s}_{i^{\prime}}$, and $i \neq i^{\prime}$ or $s \neq s^{\prime}$. A family $\vec{r}=\left(r_{b}\right)_{b \in B}$ of box replacements such that $\mathbf{A}\left(r_{b}\right) \cap \mathbf{A}\left(r_{b^{\prime}}\right)=\emptyset$ for $b \neq b^{\prime} \in B$ is applicable to the pre-term $T^{\circ}$ if $\mathbf{A}\left(T^{\circ}\right) \cap \mathbf{A}(\vec{r})=\emptyset$ and, for each $a_{i}^{b} \in \mathbf{B}\left(T^{\circ}\right), b \in B$ and $r_{b}$ is of arity at least $i$.

Definition 7.2. Let $\vec{r}$ be a $B$-indexed family of box replacements, and write $r_{b}=$ $\left(\vec{s}_{0}^{b}, \ldots, \vec{s}_{n_{b}}^{b}\right)$ for each $b \in B$. Assuming that $\vec{r}$ is applicable to the tree $S$ (resp. the 
pre-tree $S^{\circ}$ ), the substitution of $\vec{r}$ for the boxes of $S$ (resp. of $S^{\circ}$ ) is the tree $S[\vec{r}]$ (resp. the family of pre-trees $S^{\circ}\{\vec{r}\}$ ) defined by mutual induction on pre-trees and trees as follows:

$$
\begin{aligned}
& x[\vec{r}]=x \quad \lambda[\vec{r}]=\lambda \quad \mu[\vec{r}]=\mu \quad a_{0}^{b}[\vec{r}]= \begin{cases}\lambda_{b} & \text { if } \vec{s}_{0}^{b} \text { is empty } \\
!\left(\vec{s}_{0}^{b}\right) & \text { otherwise }\end{cases} \\
& \otimes\left(T_{1}, \ldots, T_{n}\right)[\vec{r}]=\otimes\left(T_{1}[\vec{r}], \ldots, T_{n}[\vec{r}]\right) \\
& \chi\left(T_{1}, \ldots, T_{n}\right)[\vec{r}]=\chi \gamma\left(T_{1}[\vec{r}], \ldots, T_{n}[\vec{r}]\right) \\
& ?\left(T_{1}^{\circ}, \ldots, T_{n}^{\circ}\right)[\vec{r}]= \begin{cases}\mu_{?}\left(T_{1}^{\circ}, \ldots, T_{n}^{\circ}\right) & \text { if } T_{1}^{\circ}\{\vec{r}\}, \ldots, T_{n}^{\circ}\{\vec{r}\} \text { is empty } \\
?\left(T_{1}^{\circ}\{\vec{r}\}, \ldots, T_{n}^{\circ}\{\vec{r}\}\right) & \text { otherwise }\end{cases} \\
& T\{\vec{r}\}=T[\vec{r}] \quad a_{i+1}^{b}\{\vec{r}\}=\vec{s}_{i+1}^{b}
\end{aligned}
$$

where each $\lambda_{b} \in \mathbf{U}_{!}$and each $\mu_{?\left(T_{1}^{\circ}, \ldots, T_{n}^{\circ}\right)} \in \mathbf{U}_{\text {? }}$ is chosen fresh (not in $\mathbf{A}(S)$ nor $\mathbf{A}\left(S^{\circ}\right)$ nor $\mathbf{A}(\vec{r}))$ and unique. ${ }^{10}$

We are now ready to introduce the expansion of MELL nets depicted in Figure $1 .{ }^{11}$ During the construction, we need to track the conclusions of copies of boxes, in order to collect copies of auxiliary ports in the external ?-links: this is the role of the intermediate notion of pre-Taylor expansion.

First, recall that we write $T_{b}, S_{b, 1}^{\circ}, \ldots, S_{b, \mathbf{a r}(b)}^{\circ}$ for the trees of $\Theta(b)$ that are respectively mapped to $a_{0}^{b}, a_{1}^{b}, \ldots, a_{\operatorname{ar}(b)}^{b}$. Also, in this case, let us write $\vec{S}_{b}^{\circ}=\left(S_{b, 1}^{\circ}, \ldots, S_{b, \operatorname{ar}(b)}^{\circ}\right)$.

Definition 7.3. Given a closed pre-net $P^{\circ}=\left(\Theta ; \vec{C} ; \vec{S}^{\circ} ; \jmath\right)$, a pre-Taylor expansion of $P^{\circ}$ is any pair $(p, f)$ of a resource net $p=\left(\vec{c} ; \vec{t} ; \jmath_{p}\right)$, together with a function $f: \vec{t} \rightarrow \overrightarrow{S^{\circ}}$ such that $f^{-1}(T)$ is a singleton whenever $T \in \vec{S}^{\circ}$ is a tree, obtained as follows:

- for each $b \in B_{\Theta}$, fix a number $k_{b} \geq 0$ of copies;

- for $1 \leq j \leq k_{b}$, fix inductively a pre-Taylor expansion $\left(p_{j}^{b}, f_{j}^{b}\right)$ of $\Theta(b)$, renaming the atoms so that the sets $\mathbf{A}\left(p_{j}^{b}\right)$ are pairwise disjoint, and also disjoint from $\mathbf{A}(\vec{C}) \cup \mathbf{A}\left(\vec{S}^{\circ}\right)$;

- write $p_{j}^{b}=\left(\vec{c}_{j}^{b} ; t_{j}^{b}, \vec{s}_{j}^{b} ; j_{j}^{b}\right)$ so that $f_{j}^{b}\left(t_{j}^{b}\right)=T_{b}$;

- write $\vec{r}=\left(r_{b}\right)_{b \in B_{\Theta}}$ for the family of box replacements $r_{b}=\left(\vec{u}_{0}^{b}, \ldots, \vec{u}_{\operatorname{ar}(b)}^{b}\right)$, where $\vec{u}_{0}^{b}=\left(t_{1}^{b}, \ldots, t_{k_{b}}^{b}\right)$ and each $\vec{u}_{i}^{b}$ is an enumeration of $\bigcup_{j=1}^{k_{b}}\left(f_{j}^{b}\right)^{-1}\left(S_{b, i}^{\circ}\right)$ for $1 \leq i \leq \operatorname{ar}(b)$;

- set $\vec{t}=\vec{S} \circ\{\vec{r}\}$ and $\vec{c}=\vec{C}[\vec{r}], \vec{c}^{\prime}$ where $\vec{c}^{\prime}$ is the concatenation of the families $\vec{c}_{j}^{b}$ for $b \in B_{\Theta}$ and $1 \leq j \leq k_{b}$

- for $t \in \vec{t}$, set $f(t)=a_{i}^{b}$ if $t \in \vec{u}_{i}^{b}$ with $1 \leq i \leq \operatorname{ar}(b)$, otherwise let $f(t)$ be the tree $T \in \vec{S}^{\circ}$ such that $t=T[\vec{r}]$;

- for each $\mu \in \mathbf{U}_{-}(p), \jmath_{p}(\mu)$ is defined as follows:

- if $\mu \in \mathbf{U}_{-}\left(p_{j}^{b}\right)$ then we set $\jmath_{p}(\mu)=\jmath_{p_{j}^{b}}(\mu)$.

\footnotetext{
${ }^{10}$ So, formally, this construction should be parametrized by suitable injections $\left\{a_{0}^{b} \in \mathbf{T}\left(S^{\circ}\right)\right\} \rightarrow \mathbf{U}_{!}$and $\left\{?\left(T_{1}^{\circ}, \ldots, T_{n}^{\circ}\right) \in \mathbf{T}\left(S^{\circ}\right)\right\} \rightarrow \mathbf{U}_{?}$ to ensure this linearity constraint. We keep this implicit in the following, but will rely on the fact that, given $t \in \mathbf{T}\left(S^{\circ}\{\vec{r}\}\right)$, one can recover unambiguously one of the following: either $T \in \mathbf{T}\left(S^{\circ}\right)$ such that $t=T[\vec{r}]$; or $b$ and $j$ such that $t \in \mathbf{T}\left(\vec{s}_{j}^{b}\right)$.

${ }^{11}$ More extensive presentations of the Taylor expansion of MELL nets exist in the literature, in various styles [PT09, GPTdF16, dC16, among others]. Our only purpose here is to introduce sufficient notations to present our analysis of the jump degree and the length of paths in $\mathcal{T}(P)$ w.r.t. the size of $P$.
} 
- if $\mu=\mu_{?\left(T_{1}^{\circ}, \ldots, T_{n}^{\circ}\right)}$ then each $T_{i}^{\circ}\{\vec{r}\}$ is empty; then we select any $i \in\{1, \ldots, n\}$ and set $\jmath_{p}(\mu)=a_{0}^{b}[\vec{r}]$ where $b \in B_{\Theta}$ is the box such that $T_{i}^{\circ}=a_{j}^{b}$ for some $1 \leq j \leq \operatorname{ar}(b)$;

- otherwise $\mu \in \mathbf{U}_{-}\left(\vec{C} ; \vec{S}^{\circ}\right.$ ), and then we set $\jmath_{p}(\mu)=\jmath(\mu)[\vec{r}]$ (note that $\jmath(\mu)$ is a tree so this is a valid application of Definition 7.2).

The Taylor expansion of a net $P$ is then $\mathcal{T}(P)=\{p \mid(p, f)$ is a pre-Taylor expansion of $P\}$.

Example 7.4. Given the net $P=\left(\Theta ; ; ?\left(a_{3}^{b}\right), ?\left(a_{2}^{b}, a_{1}^{b}\right), a_{0}^{b} ; \jmath\right)$ of Figure 15 , we construct an element $p$ of $\mathcal{T}(P)$ as follows. First, we take two copies of box $b$, fixing $k_{b}=2$. Recall that $\Theta(b)=\left(\Theta^{\prime} ;\left\langle a_{0}^{b^{\prime}} \mid \mu\right\rangle ; \bar{y}, y, a_{1}^{b^{\prime}}, ?\left(a_{2}^{b^{\prime}}\right) ; \jmath^{\prime}\right)$ where $B_{\Theta^{\prime}}=\left\{b^{\prime}\right\}$. Hence, to construct $\left(p_{j}^{b}, f_{j}^{b}\right)$ we must first fix a number $k_{b^{\prime}, j}$ of copies of the box $b^{\prime}$ : we set $k_{b^{\prime}, 1}=0$ and $k_{b^{\prime}, 2}=1$, and it remains to select a single pre-Taylor expansion $\left(p^{\prime}, f^{\prime}\right)$ of $\Theta^{\prime}\left(b^{\prime}\right)$ for the only copy of $b^{\prime}$ in $\left(p_{2}^{b}, f_{2}^{b}\right)$. Since $\Theta^{\prime}\left(b^{\prime}\right)=\left(\Theta^{\prime \prime} ; ; \bar{x}, x, \mu^{\prime} ; \jmath^{\prime \prime}\right)$ contains no box, we must have $p^{\prime}=\left(; \bar{x}, x, \mu^{\prime} ; \jmath^{\prime \prime}\right)$ with $f^{\prime}(\bar{x})=\bar{x}, f^{\prime}(x)=x$ and $f^{\prime}\left(\mu^{\prime}\right)=\mu^{\prime}$.

Since $k_{b^{\prime}, 1}=0$, we construct $p_{1}^{b}=\left(\left\langle a_{0}^{b^{\prime}} \mid \mu\right\rangle\left\{r_{b^{\prime}, 1}\right\} ;\left(\bar{y}, y, a_{1}^{b^{\prime}}, ?\left(a_{2}^{b^{\prime}}\right)\right)\left\{r_{b^{\prime}, 1}\right\} ; \jmath_{1}^{b}\right)$ where $r_{b^{\prime}, 1}$ is the empty replacement: we obtain $p_{1}^{b}=\left(\langle\lambda \mid \mu\rangle ; \bar{y}, y, \mu^{\prime \prime}\right)$ where $\lambda \in \mathbf{U}_{\text {! }}$ and $\mu^{\prime \prime} \in \mathbf{U}_{\text {? }}$ are fresh, and we set $f_{1}^{b}(\bar{y})=\bar{y}, f_{1}^{b}(y)=y, f_{1}^{b}\left(\mu^{\prime \prime}\right)=?\left(a_{2}^{b^{\prime}}\right)$, and also $j_{1}^{b}(\mu)=\jmath^{\prime}(\mu)\left[r_{b^{\prime}, 1}\right]=y$ and $\jmath_{1}^{b}\left(\mu^{\prime \prime}\right)=a_{0}^{b^{\prime}}\left[r_{b^{\prime}, 1}\right]=\lambda$.

Having defined $\left(p^{\prime}, f^{\prime}\right)$ as above, we must set $r_{b^{\prime}, 2}=\left((\bar{x}),(x),\left(\mu^{\prime}\right)\right)$ and we define $p_{2}^{b}=\left(\left\langle a_{0}^{b^{\prime}} \mid \mu\right\rangle\left\{r_{b^{\prime}, 2}\right\} ;\left(\bar{y}, y, a_{1}^{b^{\prime}}, ?\left(a_{2}^{b^{\prime}}\right)\right)\left\{r_{b^{\prime}, 2}\right\} ; j_{2}^{b}\right)$ : we obtain $p_{2}^{b}=\left(\langle!(\bar{x}) \mid \mu\rangle ; \bar{y}, y, x, ?\left(\mu^{\prime}\right)\right)$, with $f_{2}^{b}(\bar{y})=\bar{y}, f_{2}^{b}(y)=y, f_{2}^{b}(x)=a_{1}^{b^{\prime}}$ and $f_{2}^{b}\left(?\left(\mu^{\prime}\right)\right)=?\left(a_{2}^{b^{\prime}}\right)$, and we set $\jmath_{2}^{b}(\mu)=\jmath^{\prime}(\mu)\left[r_{b^{\prime}, 2}\right]=y$ and $\jmath_{2}^{b}\left(\mu^{\prime}\right)=\jmath^{\prime \prime}\left(\mu^{\prime}\right)=x$.

We rename the atoms in both pre-Taylor expansions systematically as follows: $p_{1}^{b}=$ $\left(\left\langle\lambda_{1} \mid \mu_{1}\right\rangle ; \bar{y}_{1}, y_{1}, \mu_{1}^{\prime \prime}\right)$ and $p_{2}^{b}=\left(\left\langle!\left(\bar{x}_{2}\right) \mid \mu_{2}\right\rangle ; \bar{y}_{2}, y_{2}, x_{2}, ?\left(\mu_{2}^{\prime}\right)\right)$, also redefining $f_{1}^{b}, \jmath_{1}^{b}, f_{2}^{b}$ and $\jmath_{2}^{b}$ accordingly.

Finally, we set $\vec{c}=\left\langle\lambda_{1} \mid \mu_{1}\right\rangle,\left\langle!\left(\bar{x}_{2}\right) \mid \mu_{2}\right\rangle$ and $\vec{t}=\left(?\left(a_{3}^{b}\right), ?\left(a_{2}^{b}, a_{1}^{b}\right), a_{0}^{b}\right)\left\{r_{b}\right\}$ where $r_{b}=$ $\left(\left(\bar{y}_{1}, \bar{y}_{2}\right),\left(y_{1}, y_{2}\right),\left(x_{2}\right),\left(\mu_{1}^{\prime \prime}, ?\left(\mu_{2}^{\prime}\right)\right)\right)$. We obtain:

$$
p=\left(\left\langle\lambda_{1} \mid \mu_{1}\right\rangle,\left\langle!\left(\bar{x}_{2}\right) \mid \mu_{2}\right\rangle ; ?\left(\mu_{1}^{\prime \prime}, ?\left(\mu_{2}^{\prime}\right)\right), ?\left(x_{2}, y_{1}, y_{2}\right), !\left(\bar{y}_{1}, \bar{y}_{2}\right) ; \jmath_{p}\right)
$$

with $\jmath_{p}\left(\mu_{1}\right)=y_{1}, \jmath_{p}\left(\mu_{2}\right)=y_{2}, \jmath_{p}\left(\mu_{1}^{\prime \prime}\right)=\lambda_{1}$ and $\jmath_{p}\left(\mu_{2}^{\prime}\right)=x_{2}$, which is depicted in Figure 16 .

7.3. Paths in Taylor expansion. In the following, we fix a pre-Taylor expansion $(p, f)$ of $P^{\circ}=\left(\Theta ; \vec{C} ; \vec{S}^{\circ} ; \jmath\right)$ and we describe the structure of paths in $p$. We show that the critical case depicted in Figure 17 is maximal, so that a path of $p$ passes through at most two copies of each box of $P^{\circ}$.

Observe that

$$
\mathbf{T}(p)=\left\{T[\vec{r}] \mid T \in \mathbf{T}\left(\vec{C}, \vec{S}^{\circ}\right)\right\} \cup \bigcup_{b \in B_{\Theta}} \bigcup_{j=1}^{k_{b}} \mathbf{T}\left(p_{j}^{b}\right)
$$

(using the notations of Definition 7.3). It follows that, for each $t \in \mathbf{T}(p)$ :

- either $t$ is in a copy of a box, i.e. (up to $\alpha$-equivalence) $t \in \mathbf{T}\left(p_{j}^{b}\right)$ for some $b \in B_{\Theta}$ and $1 \leq j \leq k_{b}$, and then we say $t$ is inner and write $\beta(t)=b$ and $\iota(t)=(b, j)$;

- or there exists a unique $T \in \mathbf{T}\left(P^{\circ}\right)$ such that $t=T[\vec{r}]$, and then we say $t$ is outer, and write $t^{*}=T$. 


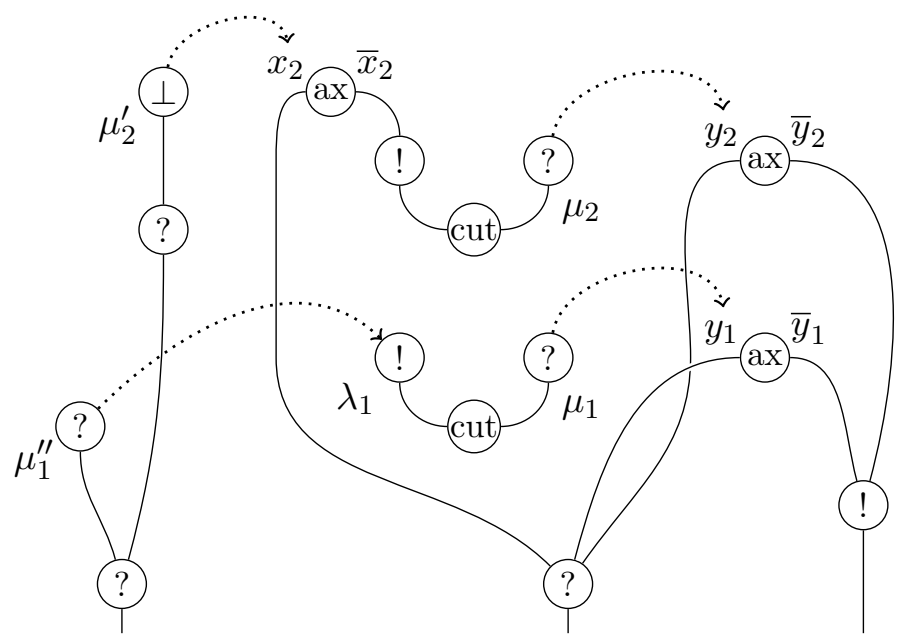

Figure 16: Representation of the resource net $p \in \mathcal{T}(P)$ where $P$ is the net of Figure 15, $k_{b}=2, k_{b^{\prime}, 1}=0$ and $k_{b^{\prime}, 2}=1$.

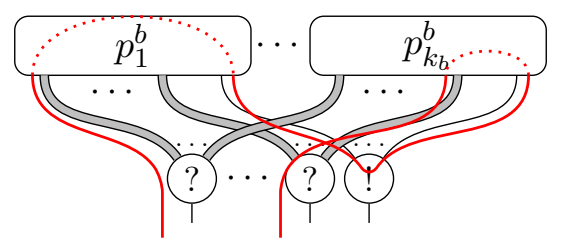

Figure 17: Box paths in Taylor expansion of $P^{\circ}$ : critical case

We further distinguish the cocontractions of $p$, i.e. the outer trees $\left(t_{1}^{b}, \ldots, t_{k_{b}}^{b}\right)$ for $b \in B_{\Theta}$, which we denote by $!_{b}$, so that $!_{b}^{*}=a_{0}^{b}$.

We say an edge $t \sim_{e}^{p, I} s$ of $p$ is an inner edge (resp. an outer edge) if $t$ and $s$ are both inner (resp. outer) trees. We say a path $\xi \in \mathbf{P}(p)$ is an inner path (resp. an outer path) if it crosses inner edges (resp. outer edges) only.

If $t \sim_{e}^{p, I} s$ is an inner edge then $\iota(t)=\iota(s)$ and we also have $t \sim_{e}^{p_{j}^{b}, I_{j}^{b}} s$ where $(b, j)=\iota(t)$ and $I_{j}^{b}$ is the restriction of $I$ to $\mathbf{U}_{-}\left(p_{j}^{b}\right)$. In this case, we also set $\beta(e)=b$ and $\iota(e)=(b, j)$. If $\xi$ is an inner path, we set $\beta(\xi)$ (resp. $\iota(\xi))$ for the common value of $\beta$ (resp. $\iota$ ) on the edges crossed by $\xi$, and we obtain:

Lemma 7.5. If $\xi$ is an inner path then $\xi \in \mathbf{P}\left(p_{j}^{b}, I_{j}^{b}\right)$ where $(b, j)=\iota(\xi)$.

The classification of the outer edges of $p$ is more delicate. First, we associate a switching $I^{*}$ of $P^{\circ}$ with each switching $I$ of $p$ as follows:

- if $I\left(\mathcal{P}\left(T_{1}[\vec{r}], \ldots, T_{n}[\vec{r}]\right)\right)=T_{i}[\vec{r}]$, we set $I^{*}\left(\not \mathcal{X}\left(T_{1}, \ldots, T_{n}\right)\right)=T_{i}$;

- if $I\left(?\left(T_{1}^{\circ}\{\vec{r}\}, \ldots, T_{n}^{\circ}\{\vec{r}\}\right)\right) \in T_{i}^{\circ}\{\vec{r}\}$, we set $I^{*}\left(?\left(T_{1}^{\circ}, \ldots, T_{n}^{\circ}\right)\right)=T_{i}^{\circ}$;

- if $?\left(T_{1}^{\circ}, \ldots, T_{n}^{\circ}\right)[\vec{r}]=\mu, T_{i}^{\circ}=a_{j}^{b}$ and $\jmath_{p}(\mu)=!_{b}$, we set $I^{*}\left(?\left(T_{1}^{\circ}, \ldots, T_{n}^{\circ}\right)\right)=T_{i}^{\circ} \cdot{ }^{12}$

\footnotetext{
${ }^{12}$ Observe that there might be several possible choices for $T_{i}^{\circ}$ so $I^{*}$ is not uniquely defined in this manner: our following constructions thus depend on the choices we make for $I^{*}$.
} 
If $t \sim_{e}^{p, I} s$ is an outer edge then, in each of the following cases, we can define an $I^{*}$-edge $e^{*}$ of $P^{\circ}$ such that $e=e^{*}[\vec{r}]$ and $t^{*} \sim_{e^{*}}^{P^{\circ}, I^{*}} s^{*}$ :

- $e$ is an axiom edge, and we set $e^{*}=e$;

- $e$ is a $\otimes$-edge, e.g. $t=\otimes\left(t_{1}, \ldots, t_{n}\right)$ and $s=t_{i}$, and we set $e^{*}=\left(t^{*}, t_{i}^{*}\right)$;

- $e$ is a 2 -edge or a ?-edge, e.g. $t=28\left(t_{1}, \ldots, t_{n}\right), s=t_{i}$ and $I(t)=s$, and we set $e^{*}=t^{*}$;

- $e=\langle t \mid s\rangle$ is a cut edge, and we set $e^{*}=\left\langle t^{*} \mid s^{*}\right\rangle$;

- $e=\mu \in \mathbf{U}_{-}\left(P^{\circ}\right) \subset \mathbf{U}_{-}(p)$ and we set $e^{*}=e$ (observe that in this case we have $\left.\jmath_{P^{\circ}}(\mu)=\jmath_{p}(\mu)^{*}\right)$.

If any of the above cases holds, we say the outer edge $e$ is superficial.

If $e$ is an outer edge that is not superficial then $e$ must be a created jump: $e=\mu \epsilon$ $\mathbf{U}_{?}(p) \backslash\left(\mathbf{U}_{?}\left(P^{\circ}\right) \cup \bigcup_{b \in B_{\Theta}} \bigcup_{j=1}^{k_{b}} \mathbf{U}_{?}\left(p_{j}^{b}\right)\right)$. If, e.g., $t=\mu$, then we can write $t^{*}=?\left(T_{1}^{\circ}, \ldots, T_{n}^{\circ}\right)$ and $s=\jmath_{p}(t)=!_{b}$ where $b$ is such that $I^{*}\left(t^{*}\right)=a_{i}^{b}$ with $1 \leq i \leq \operatorname{ar}(b)$. In this case, we obtain a path $\hat{e}=t^{*} \sim_{t^{*}}^{P^{\circ}, I^{*}} a_{i}^{b} \sim_{b, i}^{P^{\circ} I^{*}} a_{0}^{b}=s^{*}$.

Lemma 7.6. If $\xi$ is an outer $I$-path in $p$, then there exists an $I^{*}$-path $\xi^{*}$ in $P^{\circ}$ with $\ln \left(\xi^{*}\right) \geq \ln (\xi)$.

Proof. It is sufficient to replace each outer edge $e$ crossed by $\xi$ with:

- either $e^{*}$ if $e$ is superficial,

- or the path $\hat{e}$ or $\hat{e}^{\dagger}$ if $e$ is a created jump.

Observe indeed that if $t \sim_{e}^{p, I} s$ and $t^{\prime} \sim_{e^{\prime}}^{p, I} s^{\prime}$ are outer paths of length 1 with $e \neq e^{\prime}$ then the paths $\left(t \sim_{e}^{p, I} s\right)^{*}: t^{*} \rightsquigarrow P^{\circ}, I^{*} s^{*}$ and $\left(t^{\prime} \sim_{e^{\prime}}^{p, I} s^{\prime}\right)^{*}: t^{* *} \rightsquigarrow P^{\circ}, I^{*} s^{* *}$ thus defined are disjoint, and of length at least 1 .

Some edges are neither inner nor outer: a boundary edge is an edge $t \sim_{e}^{p, I} s$ such that $t$ is outer and $s$ is inner, in which case we set $\iota(e)=\iota(s)$. There are two kinds of boundary edges:

- the principal boundary of the box copy $(b, j)$ is the !-edge $\left(!_{b}, t_{j}^{b}\right)$;

- an auxiliary boundary e of the box copy $(b, j)$ is any ?-edge $t \sim_{t}^{p, I} s$ where $I(t)=s \in \vec{s}_{j}^{b}$ is such that $f_{j}^{b}(s)=S_{b, i}^{\circ}$ with $1 \leq i \leq \operatorname{ar}(b)$, in which case we must have $I^{*}\left(t^{*}\right)=a_{i}^{b}$, and then we write $\lceil e\rceil=i$ for the index of the corresponding auxiliary port.

We call box path any path of the form $\chi=(e) \xi\left(e^{\prime}\right)$ where $e$ and $e^{\prime}$ are boundaries and $\xi$ is an inner path: in this case, we write $\beta(\chi)=\beta(\xi)$ and $\iota(\chi)=\iota(\xi)$. Obviously, any path $\xi$ with outer endpoints is obtained as an alternation of outer paths and box paths: we can write uniquely $\xi=\xi_{0} \chi_{1} \xi_{1} \cdots \chi_{n} \xi_{n}$ where each $\xi_{i}$ is an outer path, and each $\chi_{i}$ is a box path.

Let $\chi=(e) \xi\left(e^{\prime}\right): t \rightsquigarrow p s$ be a box path with $\iota(\chi)=(b, j)$. Since $e \neq e^{\prime}$, then at most one of $e$ and $e^{\prime}$ is the principal boundary, and if both $e$ and $e^{\prime}$ are auxiliary boundaries, then we must have $\lceil e\rceil \neq\left\lceil e^{\prime}\right\rceil$ : indeed $e$ is the only ?-edge whose premises include $a_{\lceil e\rceil}^{b}\{\vec{r}\}$. We can thus define $\chi^{*}: t^{*} \rightsquigarrow p^{\circ} s^{*}$ as follows:

- if $e$ and $e^{\prime}$ are auxiliary boundaries then $\chi^{*}=t^{*} \sim_{t^{*}} a_{\lceil e\rceil}^{b} \sim_{b,\lceil e\rceil} a_{0}^{b} \sim_{b,\left\lceil e^{\prime}\right\rceil} a_{\left\lceil e^{\prime}\right\rceil}^{b} \sim_{s^{*}} s^{*}$

- otherwise, e.g. $e^{\prime}$ is principal and we set $\chi^{*}=t^{*} \sim_{t^{*}} a_{\lceil e\rceil}^{b} \sim_{b,\lceil e\rceil} a_{0}^{b}=s^{*}$.

Lemma 7.7. Assume $\xi=\xi_{0} \chi_{1} \xi_{1} \cdots \chi_{n} \xi_{n}: t \rightsquigarrow p, I s$ where each $\xi_{i}$ is an outer path, and each $\chi_{i}$ is a box path. Then, setting $\xi^{*}=\xi_{0}^{*} \chi_{1}^{*} \xi_{1}^{*} \cdots \chi_{n}^{*} \xi_{n}^{*}$ we obtain $\xi^{*}: t^{*} \rightsquigarrow P^{\circ}, I^{*} s^{*}$. Moreover, if $\beta\left(\chi_{i}\right)=\beta\left(\chi_{j}\right)=b$ and $i<j$, then $j=i+1, \xi_{i}=\epsilon_{!_{b}}$, and $\iota\left(\chi_{i}\right) \neq \iota\left(\chi_{j}\right)$. 
Proof. We have already observed in the proof of Lemma 7.6 that if $\xi$ and $\xi^{\prime}$ are disjoint outer paths then $\xi^{*}$ and $\xi^{\prime *}$ are also disjoint. Similarly, if $\xi$ is outer and $\chi$ is a box path, it follows directly from the definitions that $\xi^{*}$ and $\chi^{*}$ are disjoint. And if $\chi$ and $\chi^{\prime}$ are box paths with disjoint boundaries, again $\chi^{*}$ and $\chi^{*}$ are disjoint paths by construction. It follows that, if $\xi=\xi_{0} \chi_{1} \xi_{1} \cdots \chi_{n} \xi_{n}: t \rightsquigarrow p, I s$, each $\xi_{i}$ is an outer path, and each $\chi_{i}$ is a box path, then the concatenation $\xi^{*}=\xi_{0}^{*} \chi_{1}^{*} \xi_{1}^{*} \cdots \chi_{n}^{*} \xi_{n}^{*}$ is well defined.

Write $\chi_{i}=\left(e_{i}\right) \xi_{i}^{\prime}\left(e_{i}^{\prime}\right): t_{i} \rightsquigarrow s_{i}$ for $1 \leq i \leq n$. Assume $\beta\left(\chi_{i}\right)=\beta\left(\chi_{j}\right)=b$, and moreover $\beta\left(\chi_{k}\right) \neq b$ for $i<k<j$. We obtain a path $\xi^{\prime}=\left(\xi_{i} \chi_{i+1} \cdots \xi_{j-1}\right)^{*}: s_{i}^{*} \rightsquigarrow p^{\circ} t_{j}^{*}$ : by construction, $\xi^{\prime}$ does not cross any box edge $(b, l)$ for $1 \leq l \leq \operatorname{ar}(b)$. If $\left(e_{i}^{\prime}\right)$ and $\left(e_{j}\right)$ were both auxiliary, we could form a cycle $\xi^{\prime}\left(t_{j}^{*} \sim_{t_{j}^{*}} a_{\left\lceil e_{j}\right\rceil}^{b} \sim_{b,\left\lceil e_{j}\right\rceil} a_{0}^{b} \sim_{b,\left\lceil e_{i}^{\prime}\right\rceil} a_{\left\lceil e_{i}^{\prime}\right\rceil}^{b} \sim_{s_{i}^{*}} s_{i}^{*}\right)$, since $\xi^{\prime}$ would cross neither $t_{j}^{*}$ nor $s_{i}^{*}$. If, e.g., $\left(e_{i}^{\prime}\right)$ was principal and $\left(e_{j}\right)$ was auxiliary, we could form a cycle $\xi^{\prime}\left(t_{j}^{*} \sim_{t_{j}^{*}} a_{\left\lceil e_{j}\right\rceil}^{b} \sim_{b,\left\lceil e_{j}\right\rceil} a_{0}^{b}\right)$, as $\xi^{\prime}$ would not cross $t_{j}^{*}$. So both must be principal and we have $s_{i}^{*}=t_{j}^{*}=a_{0}^{b}$ : since $P^{\circ}$ has no non empty cycle, we must have $\xi^{\prime}=\epsilon_{a_{0}^{b}}$ hence $\xi_{i} \chi_{i+1} \cdots \xi_{j-1}=\epsilon_{!_{b}}$ and then $j=i+1$ and $\xi_{i}=\epsilon_{!_{b}}$. Since $e_{i}^{\prime} \neq e_{j}$, we moreover obtain $\iota\left(\chi_{i}\right) \neq \iota\left(\chi_{j}\right)$.

It remains only to prove that, in general, we never have $\beta\left(\chi_{i}\right)=\beta\left(\chi_{j}\right)$ with $j>i+1$ : otherwise, by iterating our previous argument, we would obtain $\beta\left(\chi_{k}\right)=\beta\left(\chi_{i}\right)$ whenever $i \leq k \leq j$, and both $e_{k}$ and $e_{k}^{\prime}$ would both be principal boundaries whenever $i<k<j$.

It follows that $p$ is acyclic as soon as $P^{\circ}$ is. Indeed, if $\xi$ is a cycle in $p$ :

- either $\xi$ contains an outer tree, and we can apply Lemma 7.7 to obtain a cycle in $P^{\circ}$;

- or $\xi$ is an inner path, and we proceed inductively in $\Theta(\beta(\xi))$.

Our next result is a quantitative version of this property: not only there is no cycle in $p$ but the length of paths in $p$ is bounded by a function of $P^{\circ}$ (whereas the size of $p$ is obviously not bounded in general).

Theorem 7.8. If $p \in \mathcal{T}\left(P^{\circ}\right)$ and $\xi \in \mathbf{P}(p)$ then $\ln (\xi) \leq 2^{\operatorname{depth}\left(P^{\circ}\right)} \operatorname{size}\left(P^{\circ}\right)$.

Proof. The proof is by induction on $\operatorname{depth}\left(P^{\circ}\right)$.

First assume that $\xi=\xi_{0}\left(e_{1}\right) \chi_{1}\left(e_{1}^{\prime}\right) \xi_{1} \cdots\left(e_{n}\right) \chi_{n}\left(e_{n}^{\prime}\right) \xi_{n}$ where each $\xi_{i}$ is an outer path, and each $\left(e_{i}\right) \chi_{i}\left(e_{i}^{\prime}\right)$ is a box path. Write $\left(b_{i}, j_{i}\right)=\iota\left(\chi_{i}\right)$ : by applying the induction hypothesis to $\chi_{i} \in \mathbf{P}\left(p_{j_{i}}^{b_{i}}\right)$, we obtain $\ln \left(\chi_{i}\right) \leq 2^{\operatorname{depth}\left(\Theta\left(b_{i}\right)\right)} \operatorname{size}\left(\Theta\left(b_{i}\right)\right)$. Moreover observe that $2 n+$ $\sum_{i=0}^{n} \ln \left(\xi_{i}^{*}\right) \leq \ln \left(\xi^{*}\right) \leq \operatorname{size}_{0}\left(P^{\circ}\right)$. By Lemma 7.6, it follows that $2 n+\sum_{i=0}^{n} \ln \left(\xi_{i}\right) \leq$ $\operatorname{size}_{0}\left(P^{\circ}\right)$. We obtain:

$$
\ln (\xi)=2 n+\sum_{i=0}^{n} \ln \left(\xi_{i}\right)+\sum_{i=1}^{n} \ln \left(\chi_{i}\right) \leq \operatorname{size}_{0}\left(P^{\circ}\right)+\sum_{i=1}^{n} 2^{\operatorname{depth}\left(\Theta\left(b_{i}\right)\right)} \operatorname{size}\left(\Theta\left(b_{i}\right)\right) .
$$

By Lemma 7.7, each $b \in B_{\Theta}$ occurs at most twice in the sequence $\left(b_{1}, \ldots, b_{n}\right)$, hence we obtain:

$$
\ln (\xi) \leq \operatorname{size}_{0}\left(P^{\circ}\right)+2 \sum_{b \in B_{\Theta}} 2^{\operatorname{depth}(\Theta(b))} \operatorname{size}(\Theta(b)) .
$$

hence

$$
\ln (\xi) \leq 2^{\operatorname{depth}\left(P^{\circ}\right)}\left(\operatorname{size}_{0}\left(P^{\circ}\right)+\sum_{b \in B_{\Theta}} \operatorname{size}(\Theta(b))\right) .
$$

since $\operatorname{depth}(\Theta(b))<\operatorname{depth}\left(P^{\circ}\right)$ for each $b \in B_{\Theta}$. We conclude recalling that $\operatorname{size}\left(P^{\circ}\right)=$ $\operatorname{size}_{0}\left(P^{\circ}\right)+\sum_{b \in B_{\Theta}} \operatorname{size}(\Theta(b))$. 
The other possible cases are those of paths $\chi_{0}\left(e_{0}^{\prime}\right) \xi, \xi\left(e_{n+1}\right) \chi_{n}$ or $\chi_{0}\left(e_{0}^{\prime}\right) \xi\left(e_{n+1}\right) \chi_{n}$ where $\xi$ is as above $e_{0}^{\prime}$ and $e_{n+1}$ are boundaries and $\chi_{0}$ and $\chi_{n}$ are inner paths. Reasonning as in the proof of Lemma 7.7, we also obtain that each $b \in B_{\Theta}$ occurs at most twice in the sequence, e.g., $\left(b_{0}, \ldots, b_{n+1}\right)$, and then the proof follows similarly.

In particular, we obtain $\ln (p) \leq 2^{\operatorname{depth}\left(P^{\circ}\right)} \operatorname{size}\left(P^{\circ}\right)$, In the following lemma, we show that our measure on jumps in the Taylor expansion of $P^{\circ}$ is also entirely determined by $P^{\circ}$.

Lemma 7.9. If $p \in \mathcal{T}\left(P^{\circ}\right)$ then $\mathbf{j d}(p) \leq \operatorname{size}\left(P^{\circ}\right)$.

Proof. We show that if $t \in \mathbf{T}(p)$ then $\mathbf{j d}(t) \leq \operatorname{size}\left(P^{\circ}\right)$. The proof is, again, by induction on $\operatorname{depth}\left(P^{\circ}\right)$. If $t$ is inner with $\iota(t)=(b, j)$, then we conclude directly by applying the induction hypothesis to $p_{j}^{b}$ and $\Theta(b)$ : indeed in this case, $\jmath_{p}^{-1}(t)=\jmath_{p_{j}^{b}}^{-1}(t)$, and $\operatorname{size}(\Theta(b)) \leq \operatorname{size}\left(P^{\circ}\right)$.

So we can assume that $t$ is outer. In this case, observe from Definition 7.3 that if $\jmath_{p}(\mu)=t$ then $\mu=T[\vec{r}]$ for some $T \in \mathbf{T}\left(P^{\circ}\right)$. It follows that $\# s_{p}^{-1}(t) \leq \# \mathbf{T}\left(P^{\circ}\right) \leq \operatorname{size}\left(P^{\circ}\right)$.

7.4. Cut elimination and Taylor expansion. In resource nets [ER05], the elimination of the cut

$$
\left\langle ?\left(t_{1}, \ldots, t_{n}\right) \mid !\left(s_{1}, \ldots, s_{m}\right)\right\rangle
$$

yields the finite sum

$$
\sum_{\sigma:\{1, \ldots, n\} \stackrel{\sim}{\rightarrow}\{1, \ldots, m\}}\left\langle t_{1} \mid s_{\sigma(1)}\right\rangle, \ldots,\left\langle t_{n} \mid s_{\sigma(n)}\right\rangle .
$$

It turns out that the results of Sections 3 to 6 apply directly to resource nets: setting

$$
\left\langle ?\left(t_{1}, \ldots, t_{n}\right) \mid !\left(s_{1}, \ldots, s_{n}\right)\right\rangle \rightarrow\left\langle t_{1} \mid s_{\sigma(1)}\right\rangle, \ldots,\left\langle t_{n} \mid s_{\sigma(n)}\right\rangle
$$

for each permutation $\sigma$, we obtain an instance of multiplicative reduction, as the order of premises is irrelevant from a combinatorial point of view - this is all the more obvious because no typing constraint was involved in our argument. In other words, Corollary 6.5 also applies to the parallel reduction of resource nets. With Theorem 7.8 and Lemma 7.9 we obtain:

Corollary 7.10. If $q$ is a resource net and $P$ is an $M E L L$ net and $k \in \mathbf{N},\{p \in \mathcal{T}(P) \mid$ $\left.p \rightrightarrows^{k} q\right\}$ is finite.

As for Corollaries 6.2 and 6.5, this holds only up to $\alpha$-equivalence. And, again, it should be read keeping in mind that jumps are only an additional control structure on top of the underlying net. Indeed, if $P$ is a bare MELL net (i.e. an MELL net without a jump function) then we can define $\mathcal{T}(P)$ as a set of bare resource nets. Then, given $k \in \mathbf{N}$, a bare resource net $q$, a bare MELL net $P$, and a jump function $\jmath$ such that $(P, \jmath)$ acyclic, there are finitely many bare resource nets $p \in \mathcal{T}(P)$ such that $p \rightrightarrows^{k} q$ : it suffices to construct $\jmath_{p}$ from $\jmath$.

Beware that Corollary 7.10 depends on the acyclicity of the original MELL net. The following example shows how a cyclic net can induce infinite sets of antireducts.

Example 7.11. Let $P=\left(\Theta ;\left\langle ?\left(a_{1}^{b}\right) \mid a_{0}^{b}\right\rangle ; ; \jmath\right)$ with $\Theta(b)=\left(\Theta^{\prime} ; ; x, \bar{x} ; ; \jmath\right)$ where the domain of $\Theta^{\prime}, \jmath$ and $\jmath^{\prime}$ is empty. Then, by definition,

$$
\mathcal{T}(P)=\{p=(\langle\lambda \mid \mu\rangle ;)\} \cup\left\{p_{n}=\left(\left\langle ?\left(x_{0}, \ldots, x_{n}\right) \mid !\left(\bar{x}_{0}, \ldots, \bar{x}_{n}\right)\right\rangle ;\right) \mid n \in \mathbf{N}\right\}
$$

where $\jmath_{p}(\lambda)=\mu$. Then, for each $n \in \mathbf{N}$, we have

$$
p_{n} \rightarrow\left(\left\langle x_{0} \mid \bar{x}_{\sigma(0)}\right\rangle, \ldots,\left\langle x_{n} \mid \bar{x}_{\sigma(n)}\right\rangle ;\right)
$$



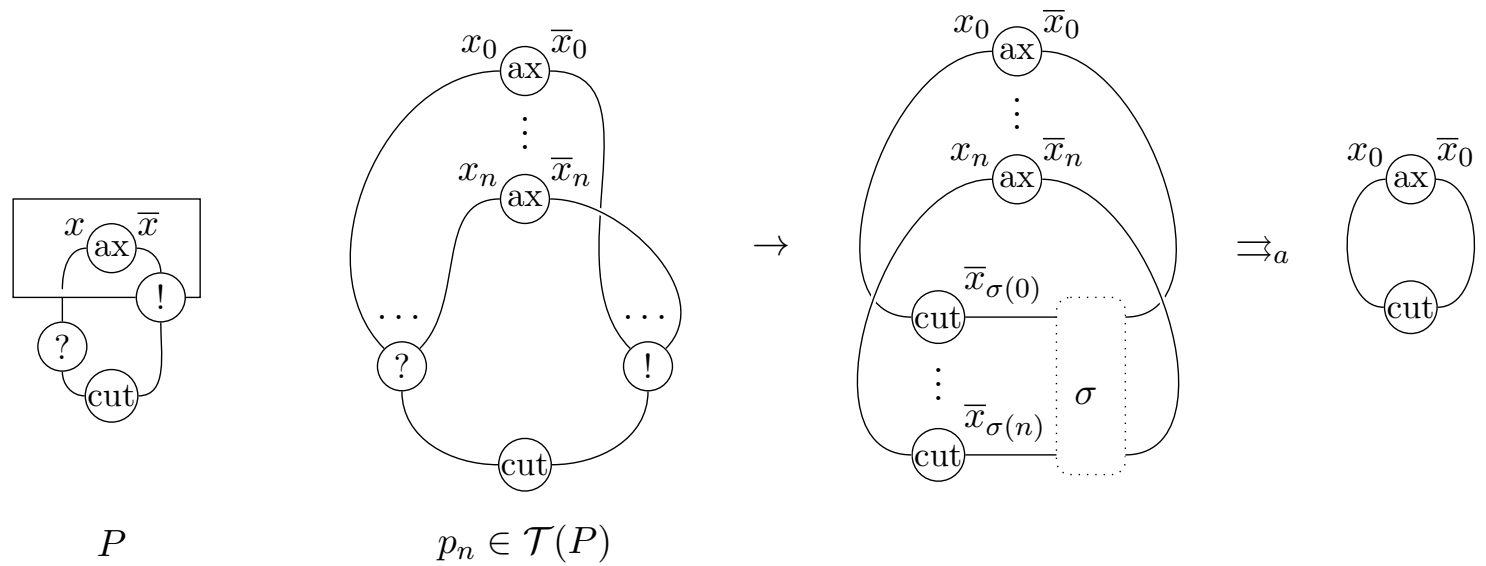

Figure 18: Resource nets $p_{n}$ of $\mathcal{T}(P)$ reducing to a single net

for each permutation $\sigma$ of $\{0, \ldots, n\}$. In particular, if we set $\sigma(i)=i+1 \bmod (n+1)$, then we obtain $p_{n} \rightarrow q_{n}=\left(\left\langle x_{0} \mid \bar{x}_{1}\right\rangle, \ldots,\left\langle x_{n} \mid \bar{x}_{0}\right\rangle ;\right) \rightrightarrows_{a}\left(\left\langle x_{0} \mid \bar{x}_{0}\right\rangle ;\right)$, and it follows that $\{q \in \mathcal{T}(P) \mid$ $\left.q \rightrightarrows^{2}\left(\left\langle x_{0} \mid \bar{x}_{0}\right\rangle ;\right)\right\}$ is infinite. This situation is illustrated in Figure 18.

\section{Conclusion}

Recall that our original motivation was the definition of a reduction relation on infinite linear combinations of resource nets, simulating cut elimination in MELL through Taylor expansion. We claim that a suitable notion is as follows:

Definition 8.1. Write $\sum_{i \in I} a_{i} p_{i} \Rightarrow \sum_{i \in I} a_{i} q_{i}$ as soon as:

- each $p_{i}$ is a resource net and each $q_{i}$ is a finite sum of resource nets such that $p_{i} \rightrightarrows q_{i}$;

- for any resource net $p,\left\{i \in I \mid p_{i}=p\right\}$ is finite;

- for any resource net $q,\left\{i \in I \mid q\right.$ is a summand of $\left.q_{i}\right\}$ is finite.

In particular, if $\sum_{i \in I} a_{i} p_{i}$ is a Taylor expansion, then Corollary 7.10 ensures that the last condition of the definition of $\Rightarrow$ is automatically valid. The details of the simulation in a quantitative setting remain to be worked out, but the main stumbling block is now over: the necessary equations on coefficients are well established, as they have been extensively studied in the various denotational models; it only remained to be able to form the associated sums directly in the syntax.

Another incentive to publish our results is the normalization-by-evaluation programme that we develop with Guerrieri, Pellissier and Tortora de Falco [CGPV17]. This approach is restricted to connected MELL proof nets, i.e. MELL proof nets without weakening, and whose switching graphs are not only acyclic but also connected: ${ }^{13}$

- in this setting, a net $P$ is entirely determined by the point of order 2 of its Taylor expansion, i.e. the unique resource net $p \in \mathcal{T}(P)$ with binary cocontractions only [GPTdF16];

- moreover, given two cut-free $\eta$-expanded nets $Q$ and $R$, both the size of the normal form of a cut between $Q$ and $R$ and the number of cut elimination steps necessary to reach it can be bounded by a function of the relational semantics of $Q$ and $R$ [dCPTdF11];

\footnotetext{
${ }^{13}$ These are sufficiently expressive to simulate the $\lambda I$-calculus, which is Turing-complete.
} 
- from this data, we obtain a bound on the size of the point $p_{0}$ of order 2 of the normal form of the cut, as well as a bound on the number of parallel cut elimination steps necessary to obtain $p_{0}$ from its antecedent $p$ in the Taylor expansion of the cut.

Our results in the present paper then provide a bound on the size of $p$ : to find $p$ it is then sufficient to compute the relational semantics of all the elements of the Taylor expansion of the cut whose size does not exceed this bound, and to check which one gives a semantics of order 2 ; then we can compute $p_{0}$ as the normal form of $p$, and this is sufficient to determine the normal form of the cut.

The restriction to connected nets is necessary to apply the injectivity result of Guerrieri, Pellissier and Tortora de Falco [GPTdF16], based on a fixed order of Taylor expansion. The injectivity of Taylor expansion and thus of the relational semantics of full MELL has been proved by de Carvalho [dC18]: to determine $P$ from $\mathcal{T}(P)$, this result relies on a $k$-heterogeneous expansion of $P$, i.e. an expansion of $P$ for which the number of copies of each box is a power of $k$, and those degrees of expansion are chosen pairwise distinct. For the result to apply, the value of the parameter $k$ must be sufficiently large: such a $k$ may be computed from the linear expansion of $P$, obtained by taking exactly one copy of each box; but the degrees of expansion of boxes cannot be bounded in advance, and it is thus not clear if the above normalization-by-evaluation procedure could be adapted in this setting.

Let us conclude with a remark about a possible adaptation of our results to a (maybe) more standard representation of nets, including separate derelictions and coderelictions, with a finer grained cut elimination procedure. This introduces additional complexity in the formalism but it essentially requires no new concept or technique: the difficulty in parallel reduction is to control the chains of cuts to be simultaneously eliminated, and decomposing cut elimination into finer reduction steps can only decrease the length of such chains. On the other hand, in that setting, it is well known that cut elimination alone is not enough to capture the $\beta$-reduction of $\lambda$-calculus, and it must be extended with additional rewriting rules accounting for structural identities (e.g., associativity and commutativity of contraction). The details of the Taylor expansion analysis of cut elimination up to these identities are worked out in the $\mathrm{PhD}$ thesis of the first author [Cho19, Chapter 2$]^{14}$, including the treatment of coefficients as mentioned above.

Acknowledgements. We would like to thank the anonymous referee whose keen remarks have allowed us to clarify several key aspects of our contributions.

This work also owes much to the friendly and stimulating environment provided by the International Research Network on Linear Logic ${ }^{15}$ between the French CNRS and the Italian INDAM: the first steps were actually taken on the occasion of the very first workshop of the network at Roma Tre in December 2015, dedicated to New trends in linear logic proof-nets; and successive versions were presented and discussed on various occasions organized by the IRN-LL.

\section{REFERENCES}

[CGPV17] Jules Chouquet, Giulio Guerrieri, Luc Pellissier, and Lionel Vaux. Normalization by evaluation in linear logic. In Stefano Guerrini, editor, Preproceedings of the International Workshop on Trends in Linear Logic and Applications, TLLA, September 2017.

\footnotetext{
${ }^{14}$ In French.

$15_{\text {http: //www. linear-logic.org/ }}$
} 
[Cho19] Jules Chouquet. A geometry of calculus. Theses, Université de Paris, December 2019.

[CVA18] Jules Chouquet and Lionel Vaux Auclair. An application of parallel cut elimination in unit-free multiplicative linear logic to the taylor expansion of proof nets. In Dan R. Ghica and Achim Jung, editors, 27th EACSL Annual Conference on Computer Science Logic, CSL 2018, September 4-7, 2018, Birmingham, UK, volume 119 of LIPIcs, pages 15:1-15:17. Schloss Dagstuhl Leibniz-Zentrum fuer Informatik, 2018.

[dC07] Daniel de Carvalho. Sémantiques de la logique linéaire et temps de calcul. PhD thesis, Université d'Aix-Marseille II, Marseille, France, 2007.

[dC09] Daniel de Carvalho. Execution time of lambda-terms via denotational semantics and intersection types. CoRR, abs/0905.4251, 2009.

[dC16] Daniel de Carvalho. The relational model is injective for multiplicative exponential linear logic. In Jean-Marc Talbot and Laurent Regnier, editors, 25th EACSL Annual Conference on Computer Science Logic, CSL 2016, August 29 - September 1, 2016, Marseille, France, volume 62 of LIPIcs, pages 41:1-41:19. Schloss Dagstuhl - Leibniz-Zentrum fuer Informatik, 2016.

[dC18] Daniel de Carvalho. Taylor expansion in linear logic is invertible. Logical Methods in Computer Science, 14(4), 2018.

[dCPTdF11] Daniel de Carvalho, Michele Pagani, and Lorenzo Tortora de Falco. A semantic measure of the execution time in Linear Logic. Theoretical Computer Science, 412, 042011.

[DE11] Vincent Danos and Thomas Ehrhard. Probabilistic coherence spaces as a model of higher-order probabilistic computation. Information and Computation, 209(6):966-991, 2011.

[DR89] Vincent Danos and Laurent Regnier. The structure of multiplicatives. Archive for Mathematical Logic, 28(3):181-203, 1989.

[Ehr02] Thomas Ehrhard. On Köthe sequence spaces and linear logic. Mathematical Structures in Computer Science, 12(5):579-623, 2002.

[Ehr05] Thomas Ehrhard. Finiteness spaces. Mathematical Structures in Computer Science, 15(4):615646, 2005.

[Ehr10] Thomas Ehrhard. A finiteness structure on resource terms. In Proceedings of the 25th Annual IEEE Symposium on Logic in Computer Science, LICS 2010, 11-14 July 2010, Edinburgh, United Kingdom, pages 402-410, 2010.

[Ehr14] Thomas Ehrhard. A new correctness criterion for MLL proof nets. In Joint Meeting of the Twenty-Third EACSL Annual Conference on Computer Science Logic (CSL) and the TwentyNinth Annual ACM/IEEE Symposium on Logic in Computer Science (LICS), CSL-LICS '14, Vienna, Austria, July 14 - 18, 2014, pages 38:1-38:10, 2014.

[Ehr16] Thomas Ehrhard. An introduction to differential linear logic: proof-nets, models and antiderivatives. CoRR, abs/1606.01642, 2016.

[ER03] Thomas Ehrhard and Laurent Regnier. The differential lambda-calculus. Theoretical Computer Science, 309(1-3):1-41, 2003.

[ER05] Thomas Ehrhard and Laurent Regnier. Differential interaction nets. Electr. Notes Theor. Comput. Sci., 123:35-74, 2005.

[ER08] Thomas Ehrhard and Laurent Regnier. Uniformity and the taylor expansion of ordinary lambda-terms. Theoretical Computer Science, 403(2-3):347-372, 2008.

[FM99] Maribel Fernández and Ian Mackie. A calculus for interaction nets. In Gopalan Nadathur, editor, Principles and Practice of Declarative Programming, International Conference PPDP'99, Paris, France, September 29 - October 1, 1999, Proceedings, volume 1702 of Lecture Notes in Computer Science, pages 170-187. Springer, 1999.

[Gir87] Jean-Yves Girard. Linear logic. Theoretical Computer Science, 50:1-102, 1987.

[Gir88] Jean-Yves Girard. Normal functors, power series and lambda-calculus. Annals of Pure and Applied Logic, 37(2):129, 1988.

[Gir96] Jean-Yves Girard. Proof-nets : the parallel syntax for proof-theory. In Aldo Ursini and Paolo Aglianò, editors, Logic and Algebra, number 180 in Lecture Notes in Pure and Applied Mathematics. Marcel Dekker, New York, 1996.

[GPTdF16] Giulio Guerrieri, Luc Pellissier, and Lorenzo Tortora de Falco. Computing connected proof(structure)s from their taylor expansion. In 1st International Conference on Formal Structures for 
Computation and Deduction, FSCD 2016, June 22-26, 2016, Porto, Portugal, pages 20:1-20:18, 2016.

[HH16] Willem Heijltjes and Robin Houston. Proof equivalence in MLL is pspace-complete. Logical Methods in Computer Science, 12(1), 2016.

[Laf90] Yves Lafont. Interaction nets. In Frances E. Allen, editor, Conference Record of the Seventeenth Annual ACM Symposium on Principles of Programming Languages, San Francisco, California, USA, January 1990, pages 95-108. ACM Press, 1990.

[LMMP13] Jim Laird, Giulio Manzonetto, Guy McCusker, and Michele Pagani. Weighted relational models of typed lambda-calculi. In 28th Annual ACM/IEEE Symposium on Logic in Computer Science, LICS 2013, New Orleans, LA, USA, June 25-28, 2013, pages 301-310. IEEE Computer Society, 2013.

[MS08] Ian Mackie and Shinya Sato. A calculus for interaction nets based on the linear chemical abstract machine. Electron. Notes Theor. Comput. Sci., 192(3):59-70, 2008.

[PT09] Michele Pagani and Christine Tasson. The inverse taylor expansion problem in linear logic. In Proceedings of the 24th Annual IEEE Symposium on Logic in Computer Science, LICS 2009, 11-14 August 2009, Los Angeles, CA, USA, pages 222-231, 2009.

[PTV16] Michele Pagani, Christine Tasson, and Lionel Vaux. Strong normalizability as a finiteness structure via the taylor expansion of lambda-terms. In Foundations of Software Science and Computation Structures - 19th International Conference, FOSSACS 2016, Held as Part of the European Joint Conferences on Theory and Practice of Software, ETAPS 2016, Eindhoven, The Netherlands, April 2-8, 2016, Proceedings, pages 408-423, 2016.

[Reg92] Laurent Regnier. Lambda-calcul et réseaux. PhD thesis, Université Paris 7, Paris, France, December 1992.

[Tas09] Christine Tasson. Sémantiques et syntaxes vectorielles de la logique linéaire. PhD thesis, Université Paris Diderot, Paris, France, December 2009.

[TdF00] Lorenzo Tortora de Falco. Réseaux, cohérence et expériences obsessionnelles. PhD thesis, Université Paris 7, Paris, France, 2000.

[Vau17] Lionel Vaux. Taylor expansion, $\beta$-reduction and normalization. In 26th EACSL Annual Conference on Computer Science Logic, CSL 2017, August 20-24, 2017, Stockholm, Sweden, pages 39:1-39:16, 2017. 